
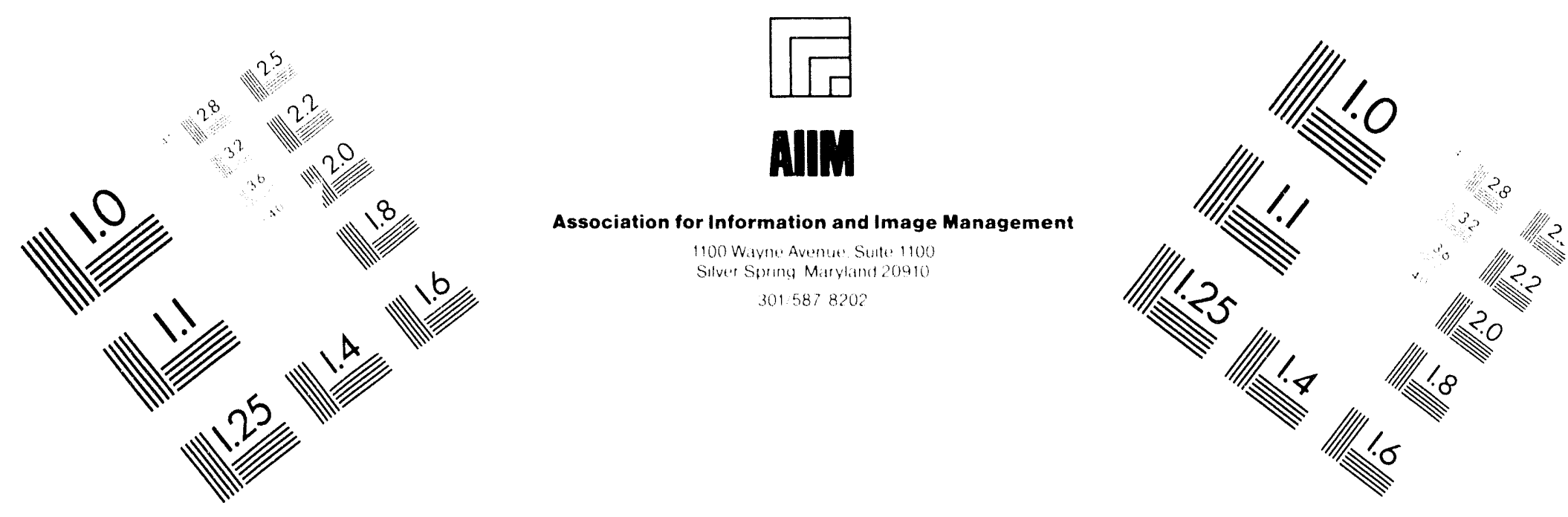

\title{
Centimeter
}

mW

Inches
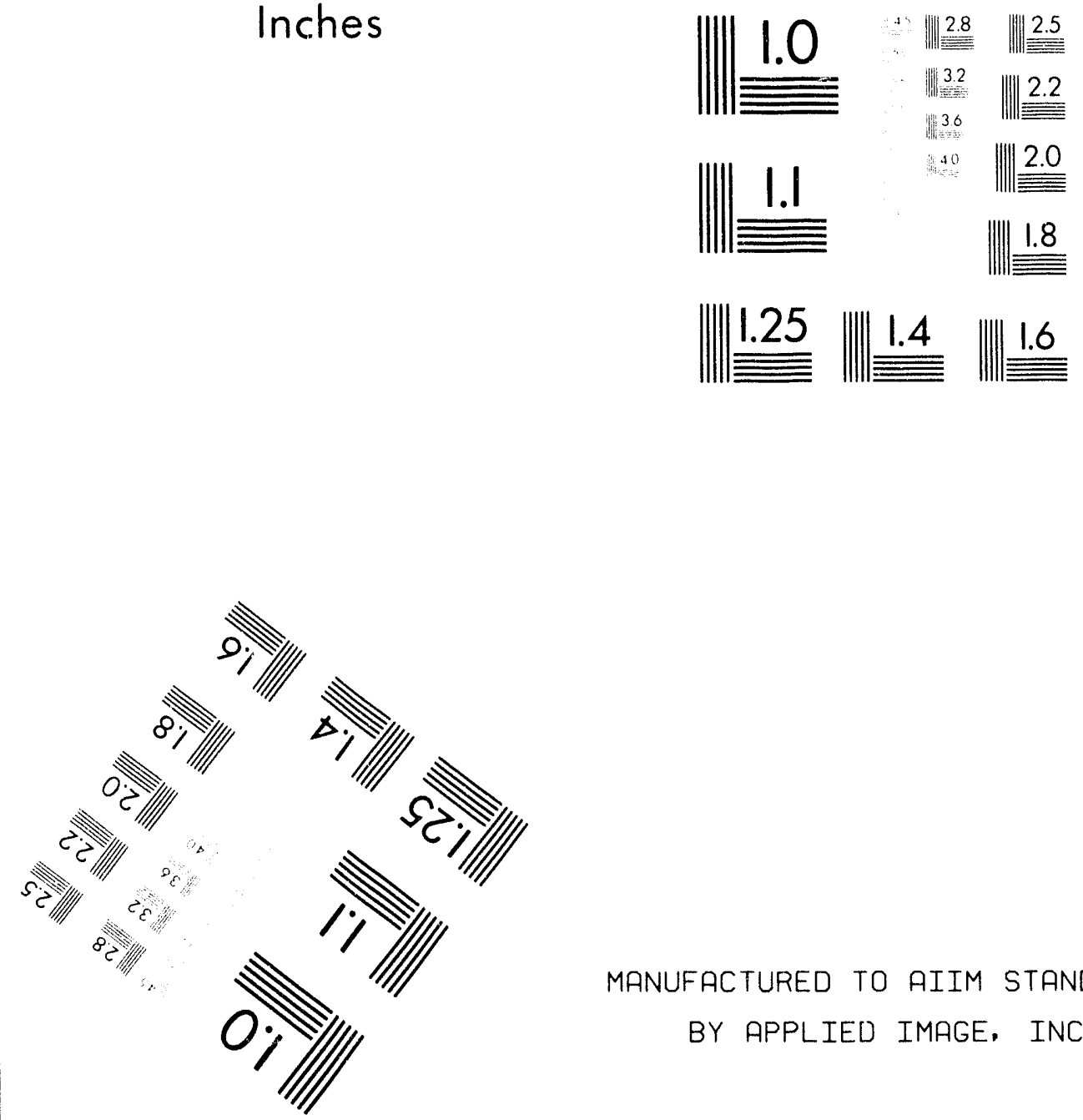

MANUFACTURED TO AIIM STANDARDS

BY APPLIED IMAGE. INC.

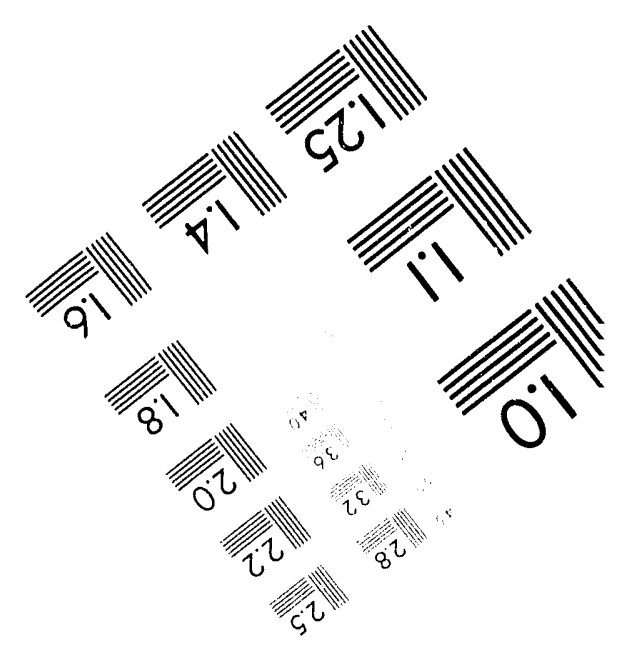



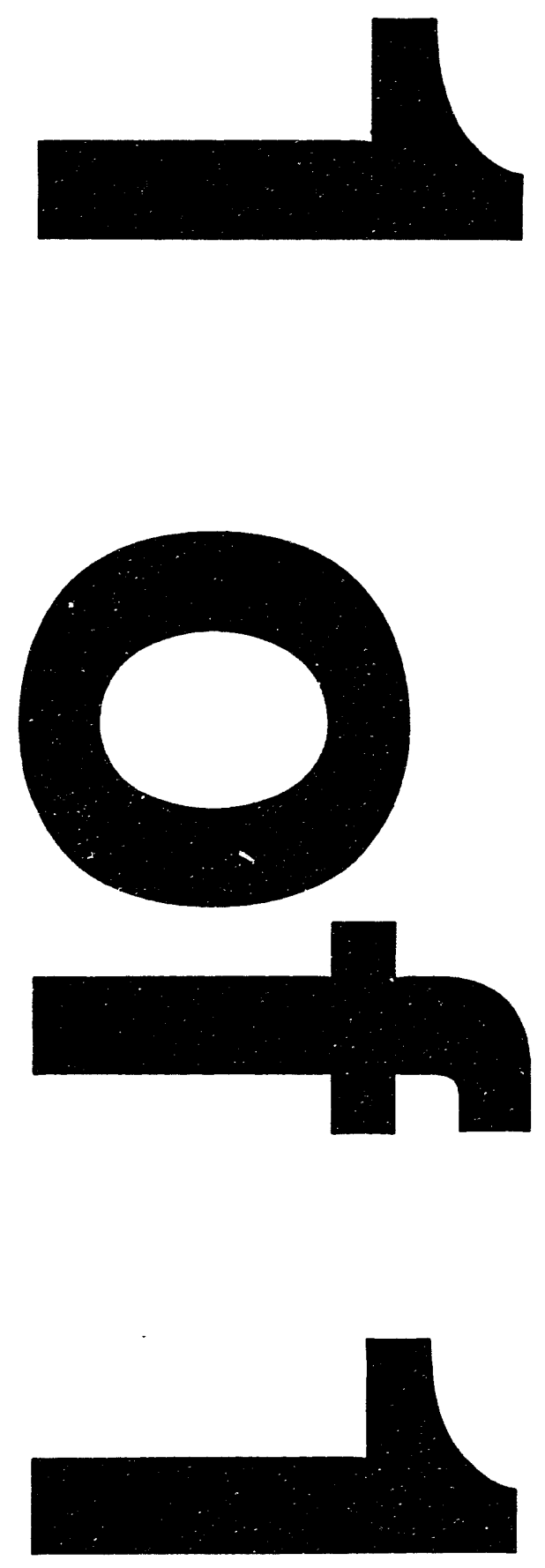
He
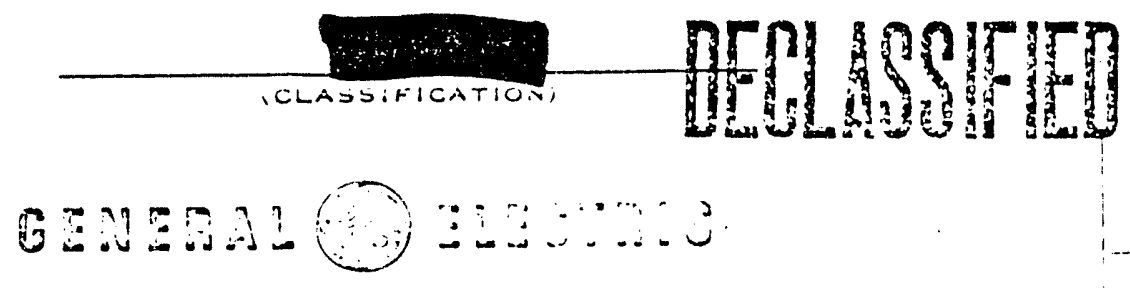

HANFORD ATOMIC PRODUCTS OPERATION - SICMLAND, WASMANETON strate ano con cor no.

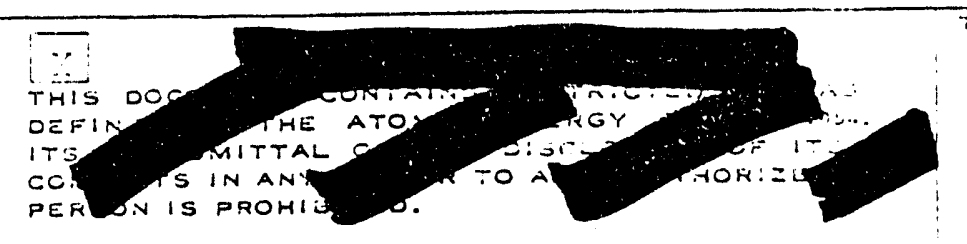

TTL:

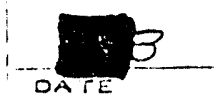

W OTHER OFFICIAL CLISSIF!EO INFORMATIO:

THIS MATERIAL CONTAINS INFORMATION AFFEC:

THE NATIONAL DEFENJE OF THE UNITED STATL:

WithiN THE MEANING of the EsPIONAGE LAW:.

TITLE 18, U.S.C. SECS. 793 AND 79A. THE TRANS-

MISSION OR REVELLTION OF WHICH IN ANY MANAZS

TO AN UNAUTHORIIZCD PERSON IS PROHIBITED UY

LAW.
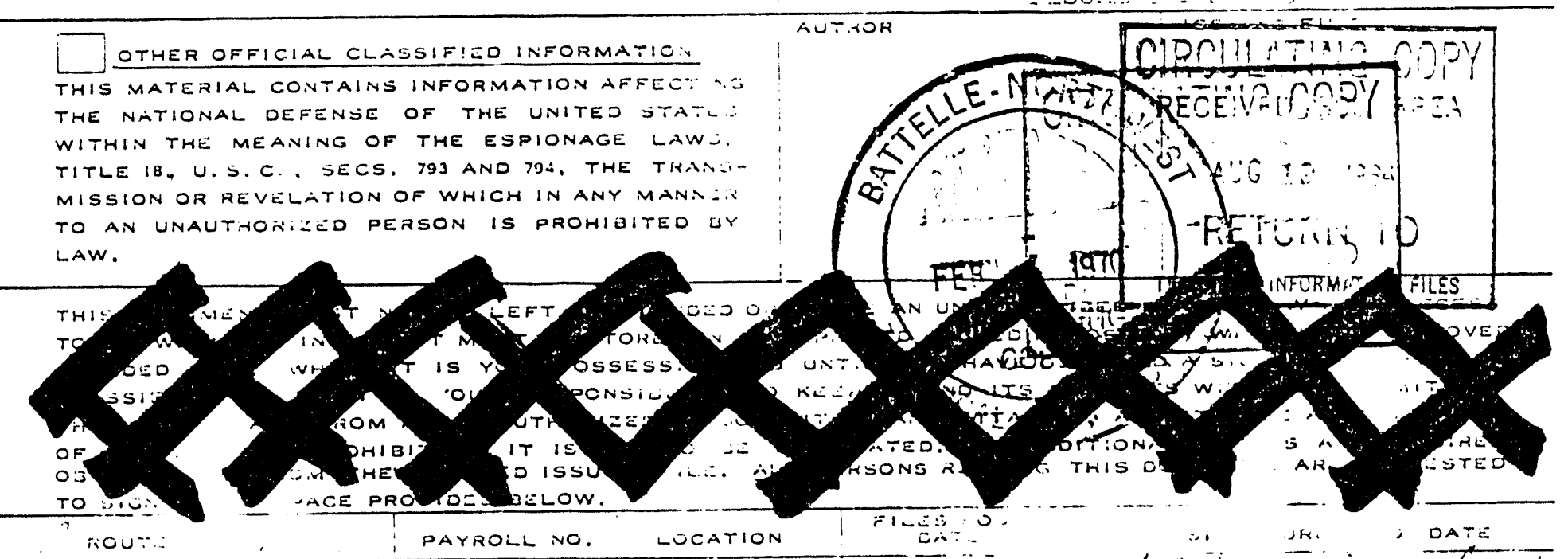

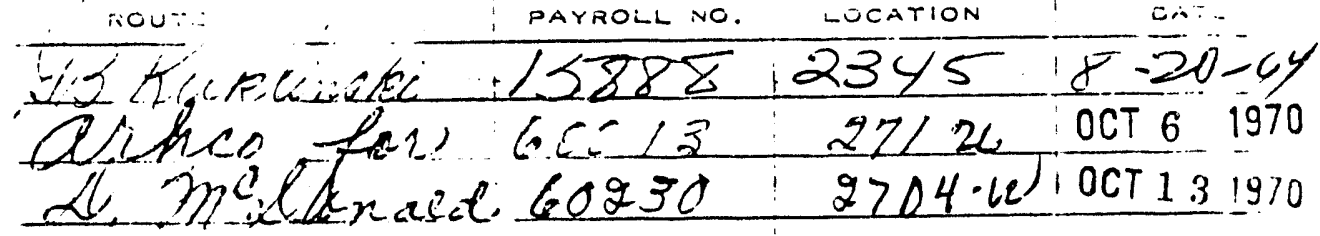

Tim $i 1-4-70$ 


\section{MPI MPITIT]}

CONTINUOUS PAE MTION FOR HIV

\begin{tabular}{|c|c|c|c|c|c|}
\hline AS OF & $1130 / 64$ & (DATE) & THA DOCUNENT & CONSISTS OF & . NES \\
\hline AS OF & $10-12->0$ & (DATE) & THIS DOCUMENT & CONSISTS OF & GES \\
\hline AS OF & & (DATE) & THIS DOCUMENT & CONSISTS OF & $\therefore$ SES \\
\hline As or & & (DATE) & THIS DOCUME:UT & CONSISTS OF & . MEES \\
\hline AS OF & - & (DATE) & THIS DOCUMENT & CONSISTS OF & PAGES \\
\hline AS OF & & (DATE) & THIS DOCUMLNT & CONSISTS OF & PAGES \\
\hline As or & & (DATE) & THIS DOCUMENT & CONSISTS OF & HEVSS \\
\hline AS OF & & (DATE) & THIS DOCUM:NT & CONSISTS OF & PMiEs \\
\hline AS OF & & (DATE) & THIS DOCUMLNT & CONSISTS OF & pAGES \\
\hline As OF & & (DATE) & THIS DOCUMENT & CONSISTS OF & PAGES \\
\hline AS OF & & (DATE) & THI: DOCUMINT & CONSISTS OF & $\therefore$ NES \\
\hline AS OF & & (DATE) & THIS DOCUMENT & CONSISTS OF & $-A$ SES \\
\hline AS OF & & (DATE) & THIS DOCUMENT & CONSISTS OF & AGES \\
\hline AS OF & & (DATE) & THIS DOCUMINTT & CONSISTS DF & DAIIES \\
\hline AS OF & & (DATE) & THIS DOCUMENT & CONSISTS OF & PAGES \\
\hline As $O=$ & & (OATE) & Tr:IS OCOUMI-NT & OONSISTS OF & WALES \\
\hline nor & & (DATE) & ind RUUMENT & CON $=I S T S O F$ & WES \\
\hline SOF & & (DATE) & THI W DOCUMENT & CONSISTS OF & AMES \\
\hline AS OF & & (DATE) & THIS DOCUMENT & CONSISTS OF & F.ACES \\
\hline AS OF & & (DATE) & THIS DOCUMENT & CONSISTS OF & AAEES \\
\hline AS OF & & - (DATE) & THIS DOCUMENT & CONSISTS OF & MASES \\
\hline AS OF. & & (DATE) & THIS DOCUMENT & CONSISTS OF & PAGES \\
\hline AS OF & & (DATE) & THIS DOCUMENT & CONSISTS OF & PAGES \\
\hline AS OF & & (DATE) & THIS DOCUMENT & CONSISTS OF & FAGES \\
\hline As OF & & (DATE) & THIS DOCUMENT & CONSISTS OF & PAGES \\
\hline AS OF & & (DATE) & THIS DOCUMENT & CONSISTS OH. & MAEES \\
\hline As OF & 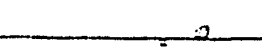 & (DATE) & THIS DOCUMENT & CONSISTS OF & PAGES \\
\hline As of & & (DATE) & THIS DOCUMENT & CONSISTS OF & PAGES \\
\hline$\triangle S$ OF & & (DATE) & Th.s so=UnENT & CONSISTS OF & PAGES \\
\hline AS OF & & (DATE) & THIS JOCUI .. NT & CONSISTS OF - & PAGES \\
\hline
\end{tabular}

THIS CONTINUOUS PAIINATION FORM IS TO E. USK I IN ACCUN.ULATING CLASSIFIED WONKING

PAPERS IN A BINDE. ISSUED AND CONTROL-IJ BY O.. ASEIFIED FILES. 


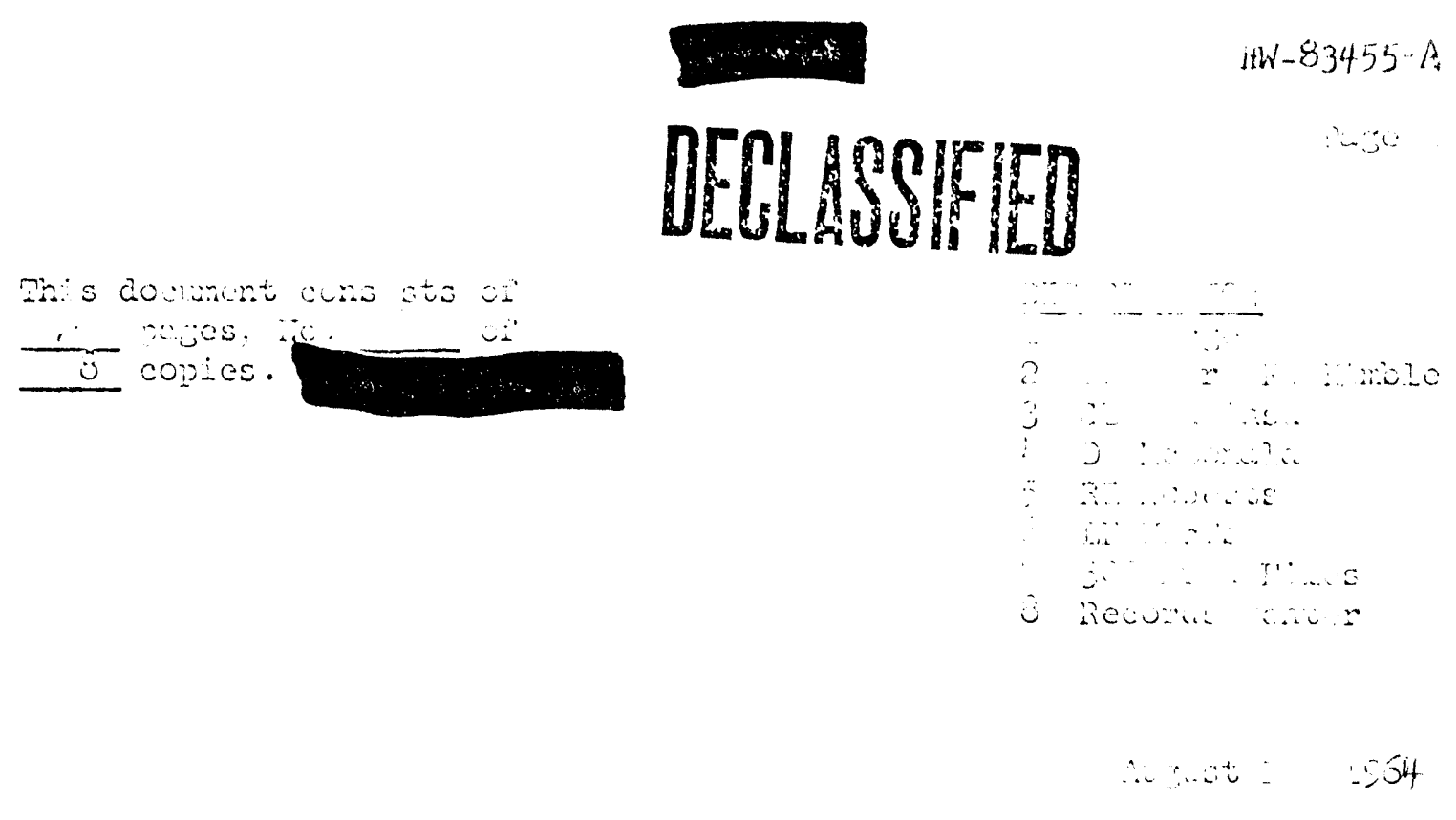

MERAL EALUSE MD ME MODUCTIOH REPOR
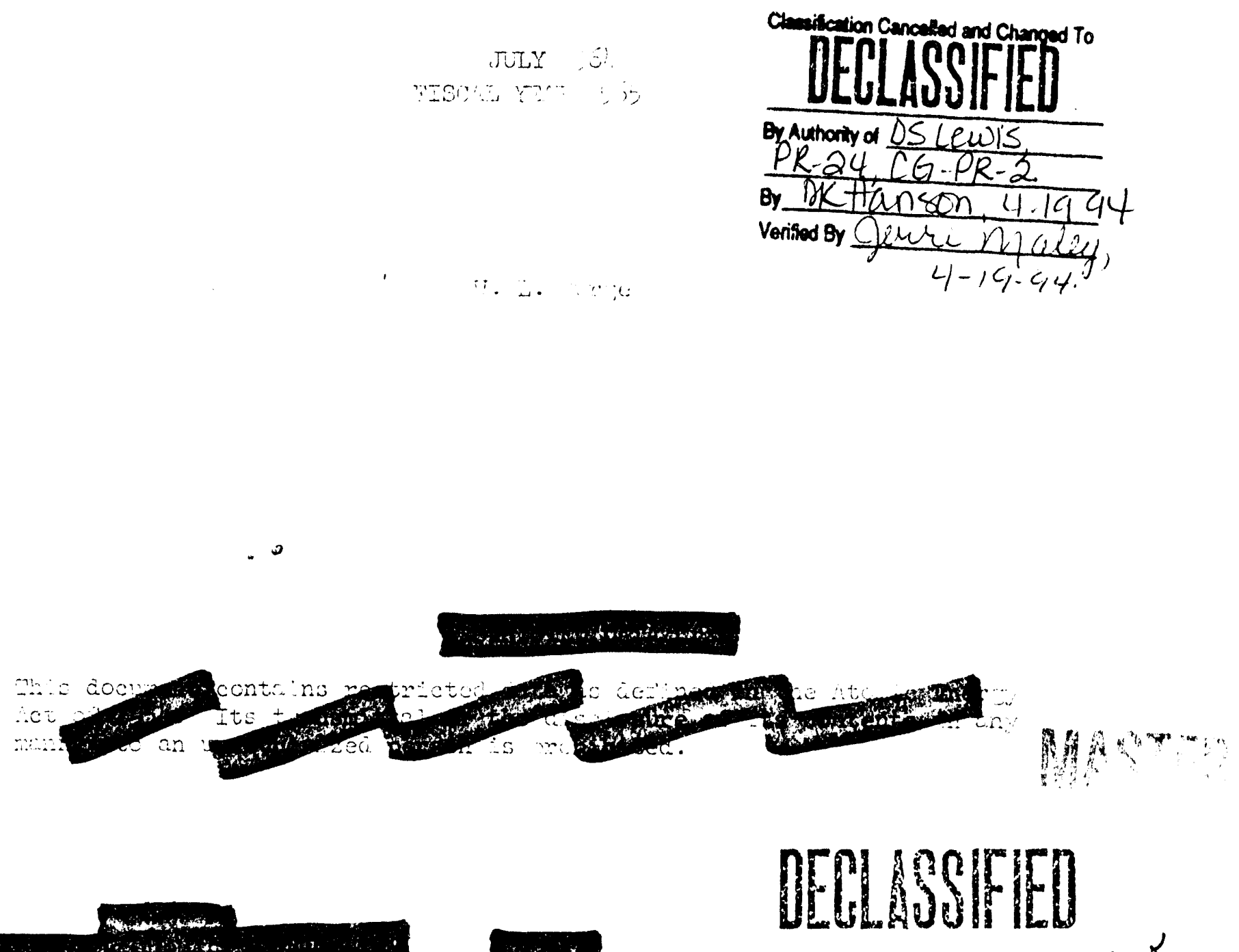


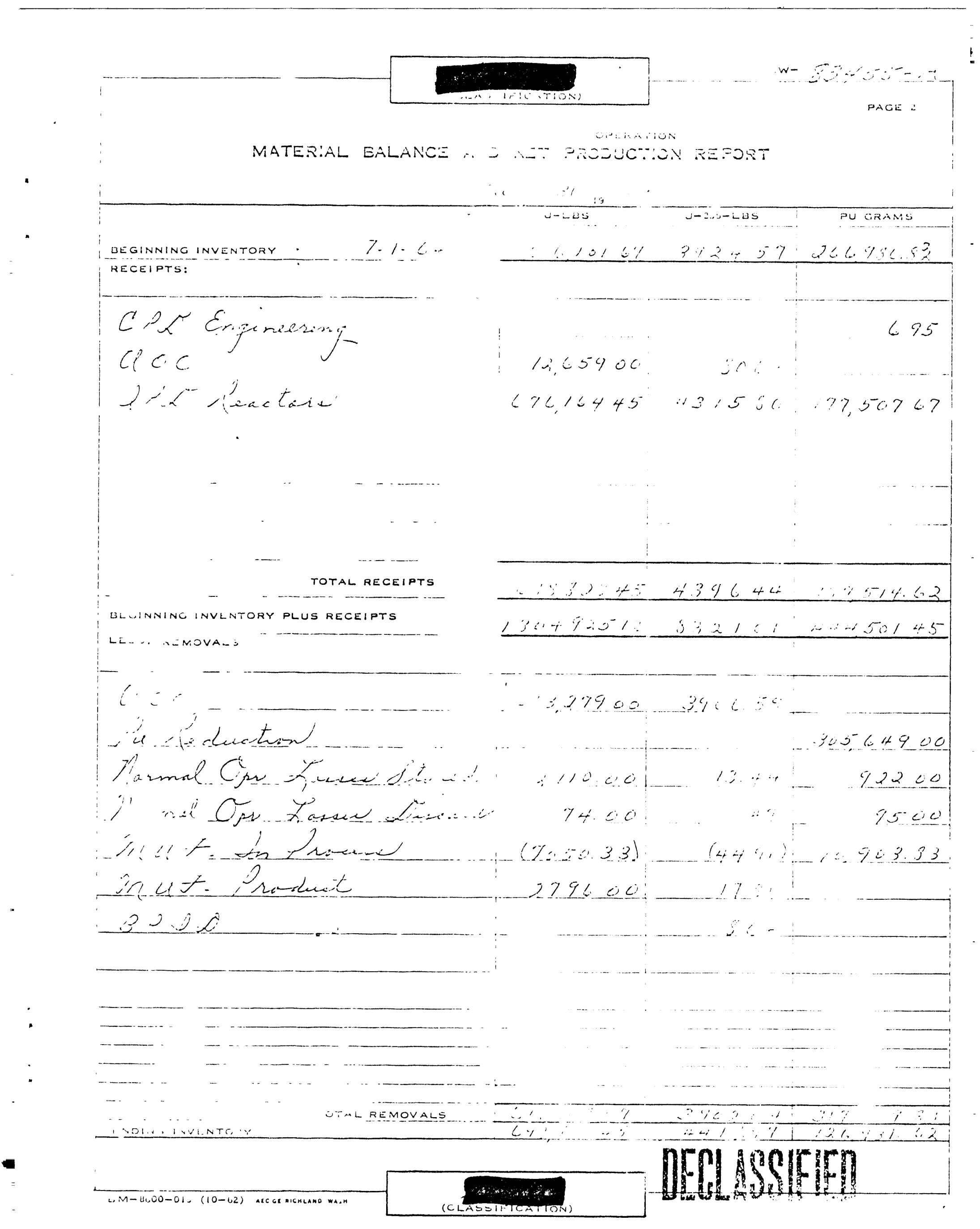


MARTERIAL EALADEニ
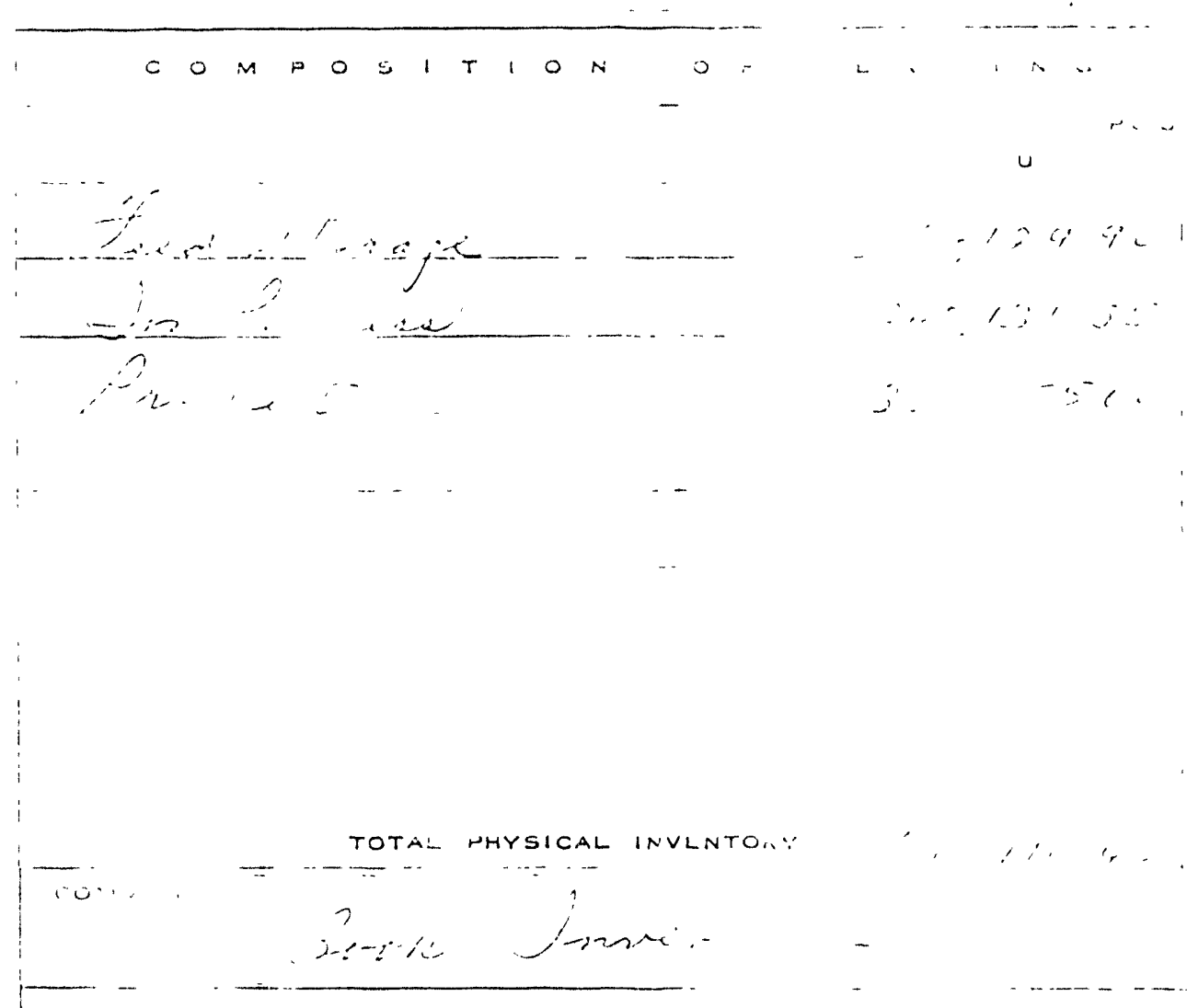

HLL . AUM

ïris
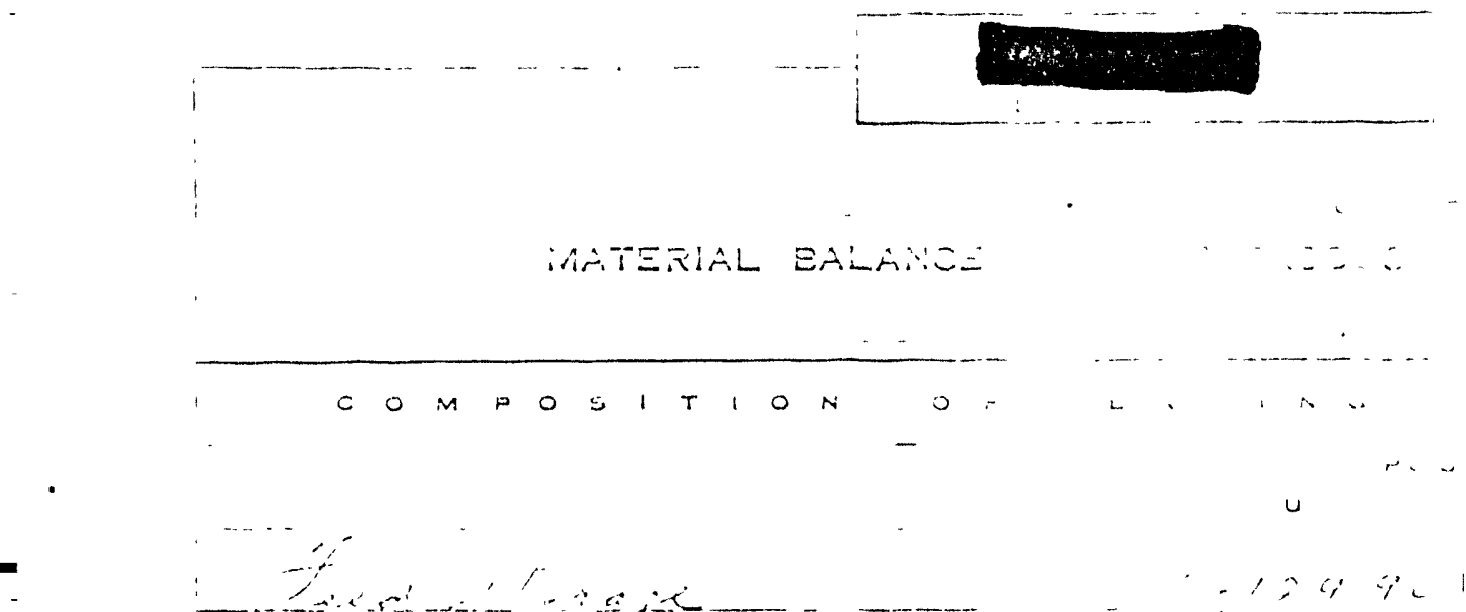


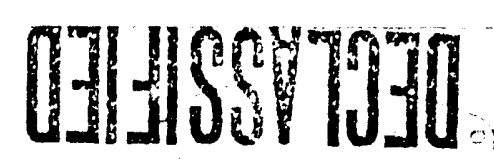

\section{$\Rightarrow$}

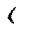

$\sqrt{1}$

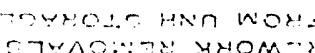

$$
\text { Noidongorel Han: }
$$

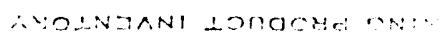

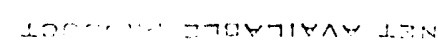

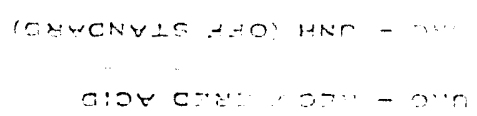

: worat SAd:

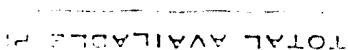
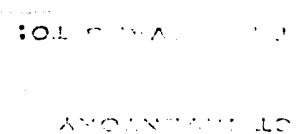

w $\cap 1$

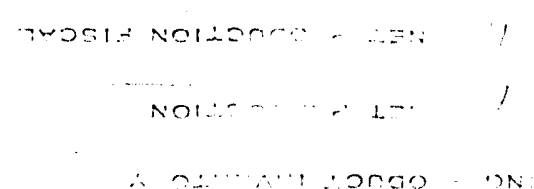

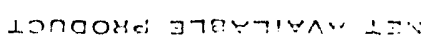

7พ-20: nก:

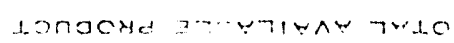

$7 \div 10 \times \cdots$

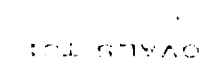

A $⿻ \therefore \cdots \cdots+1$

$\equiv 0+40-7=$ 


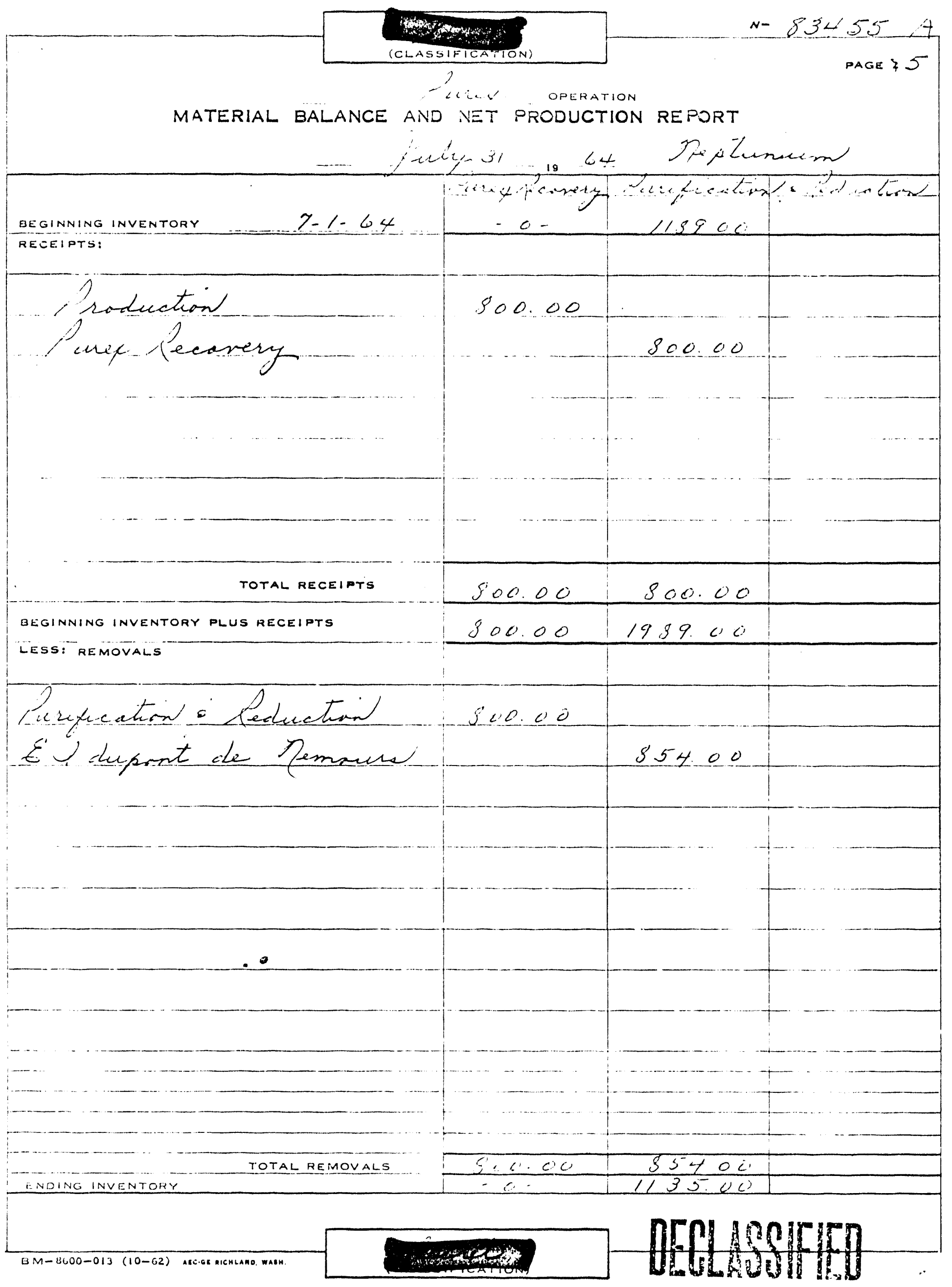




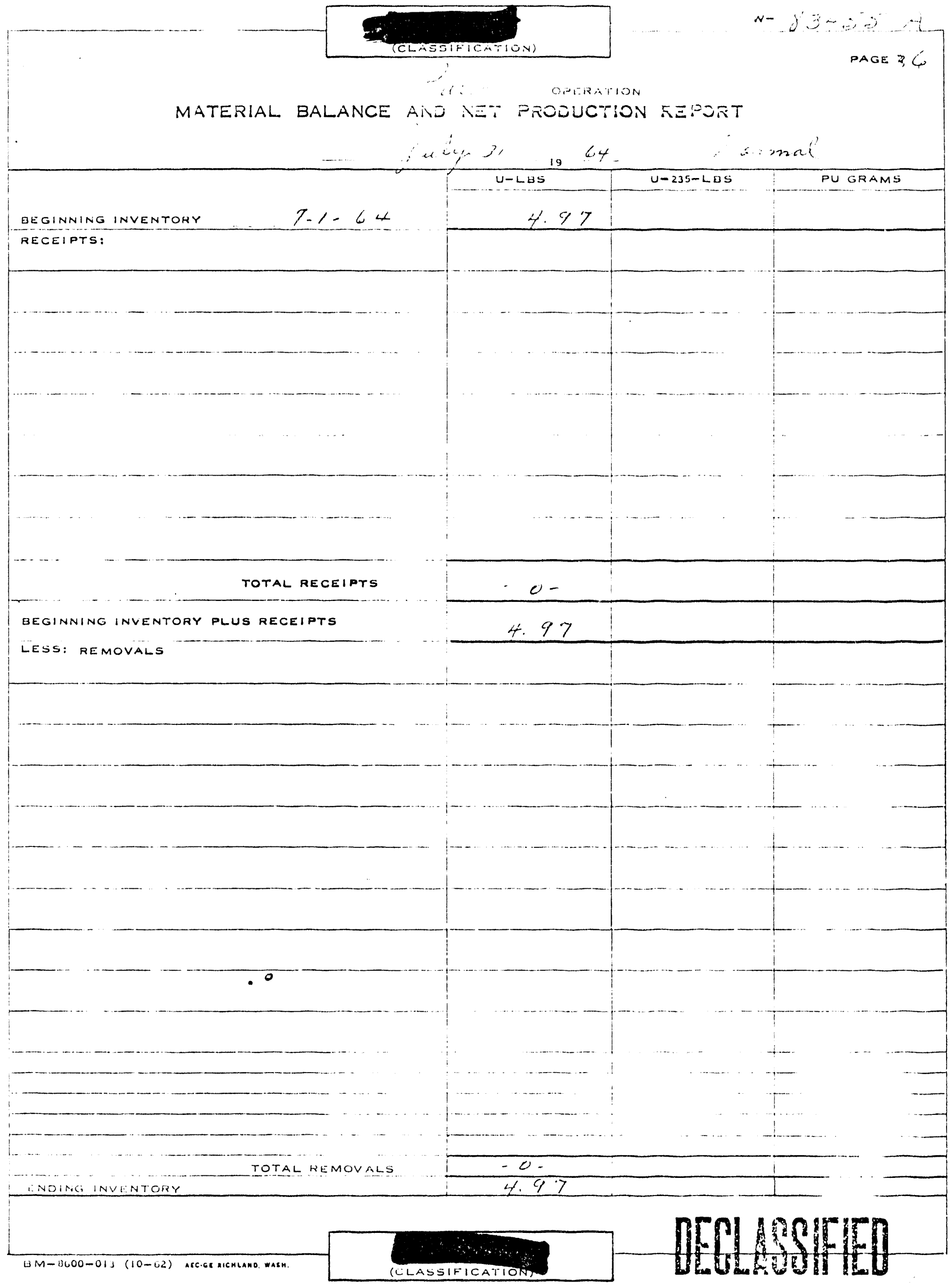




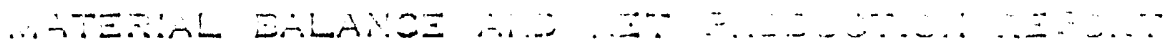

DEGINNING INVENTORY $2^{2}-1-6: 2$

RECEIPTS:

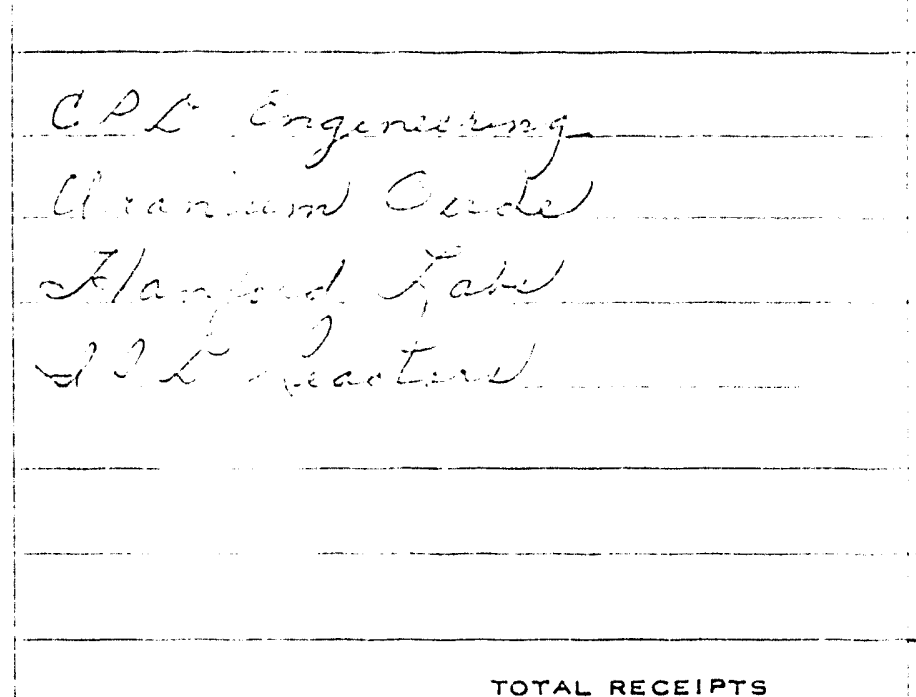

TOTAL RECEIPTS

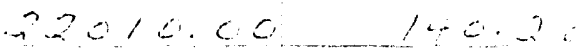

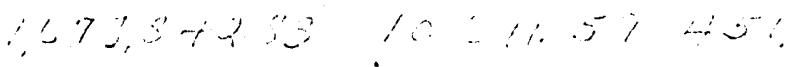

BEGINNING INVENTORY PLUS RECEIPTS

LESS: REMOVALS

$17005 \div 39,2519 \%$

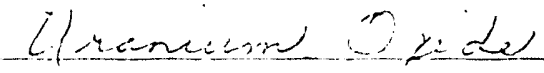

$95097<0 \quad 3 \leq y 0$

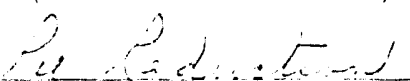

sim C-26.t"

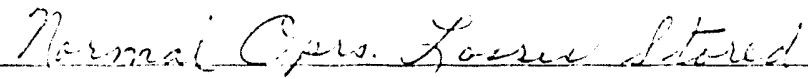

$239+5490915,92207$

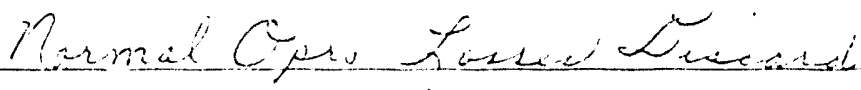

in $1 z$ zin inase

in $\therefore$ - oredures

$\hat{s}+2$

$40+8<6 \quad 0579$

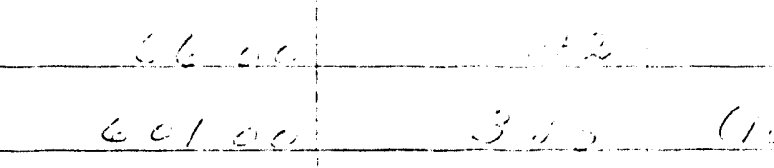

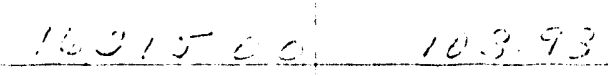

$15 \div 6$

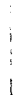



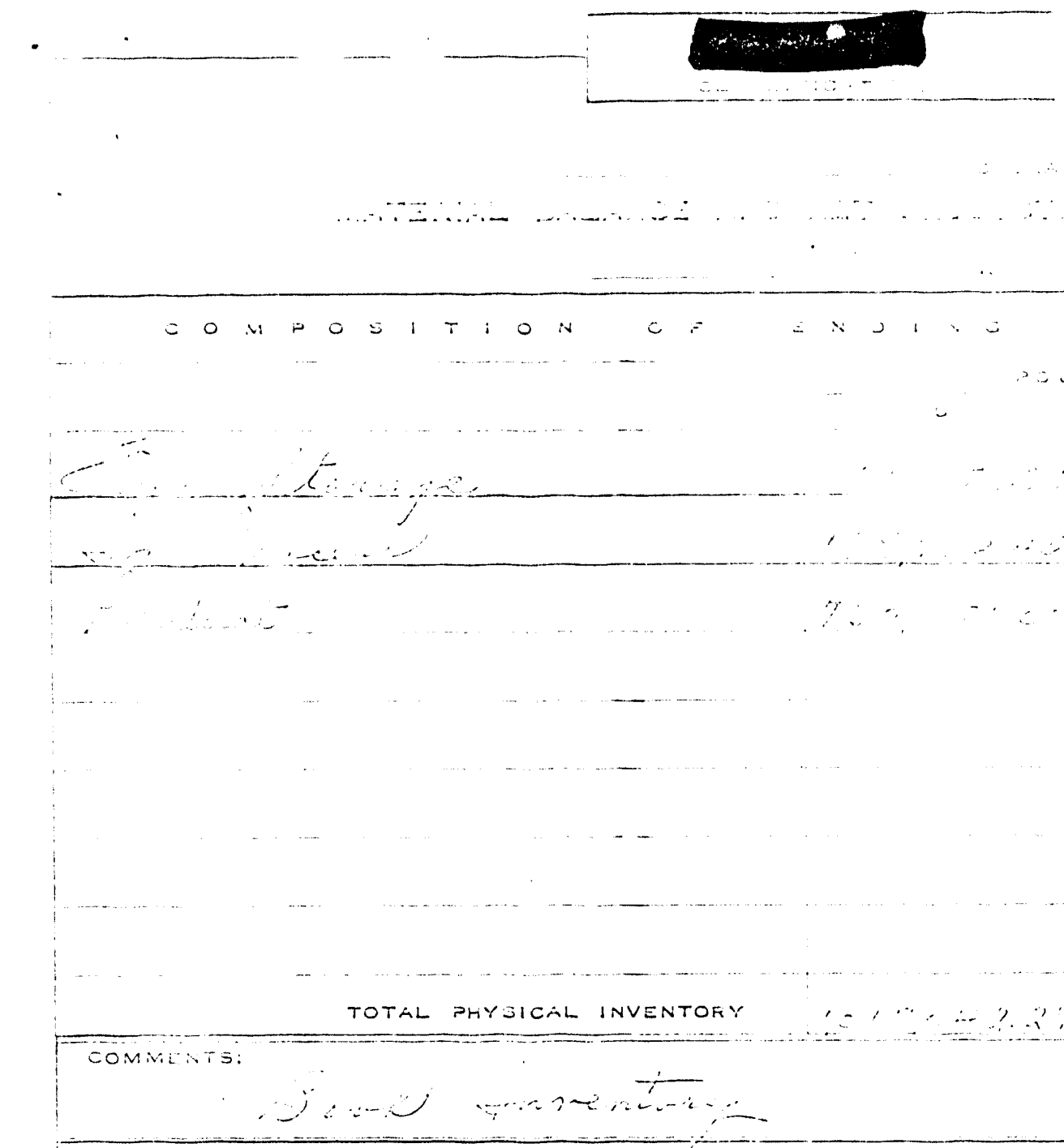

$-\therefore 1-\cdots, 00-12,: 00-62$ 


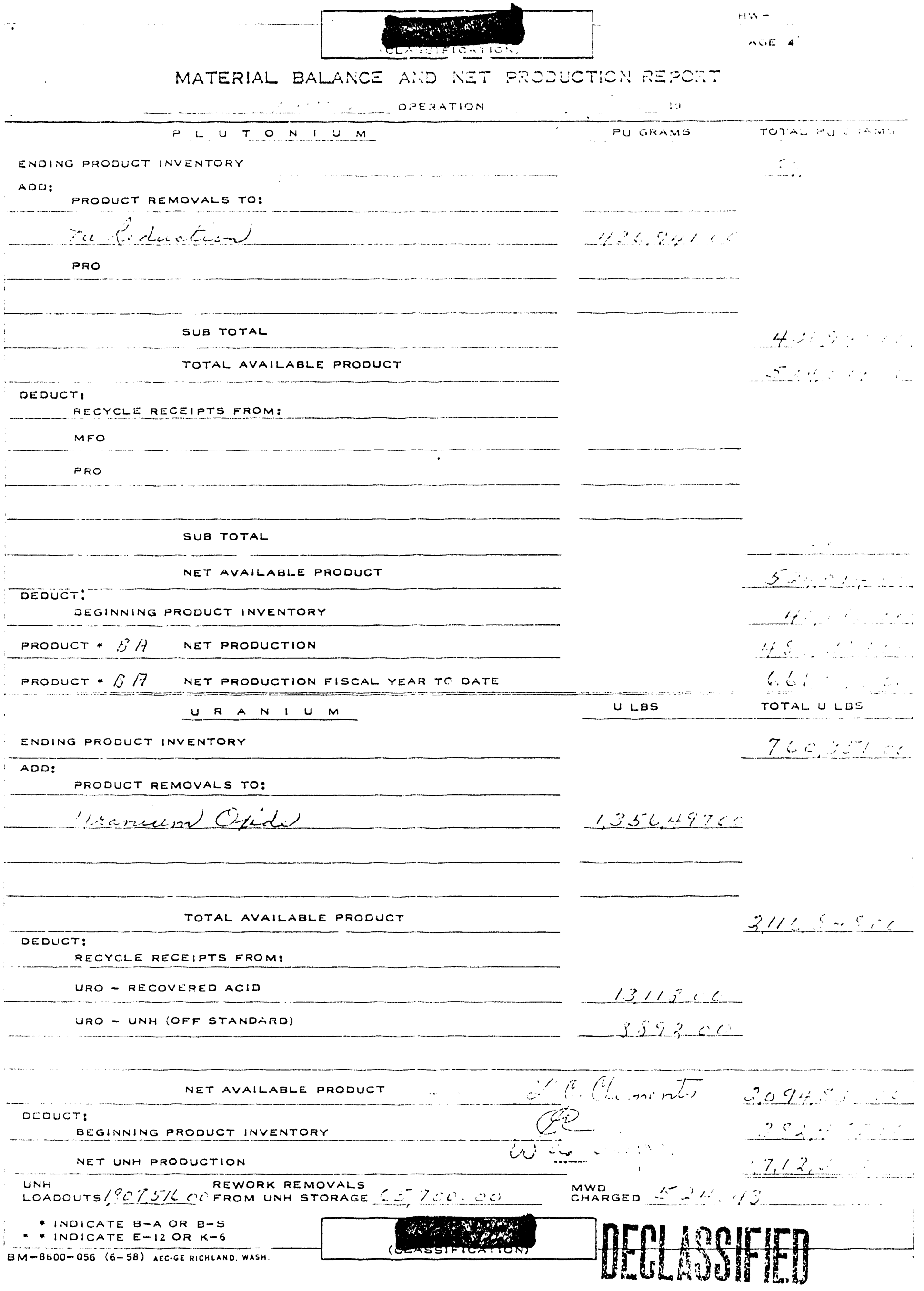




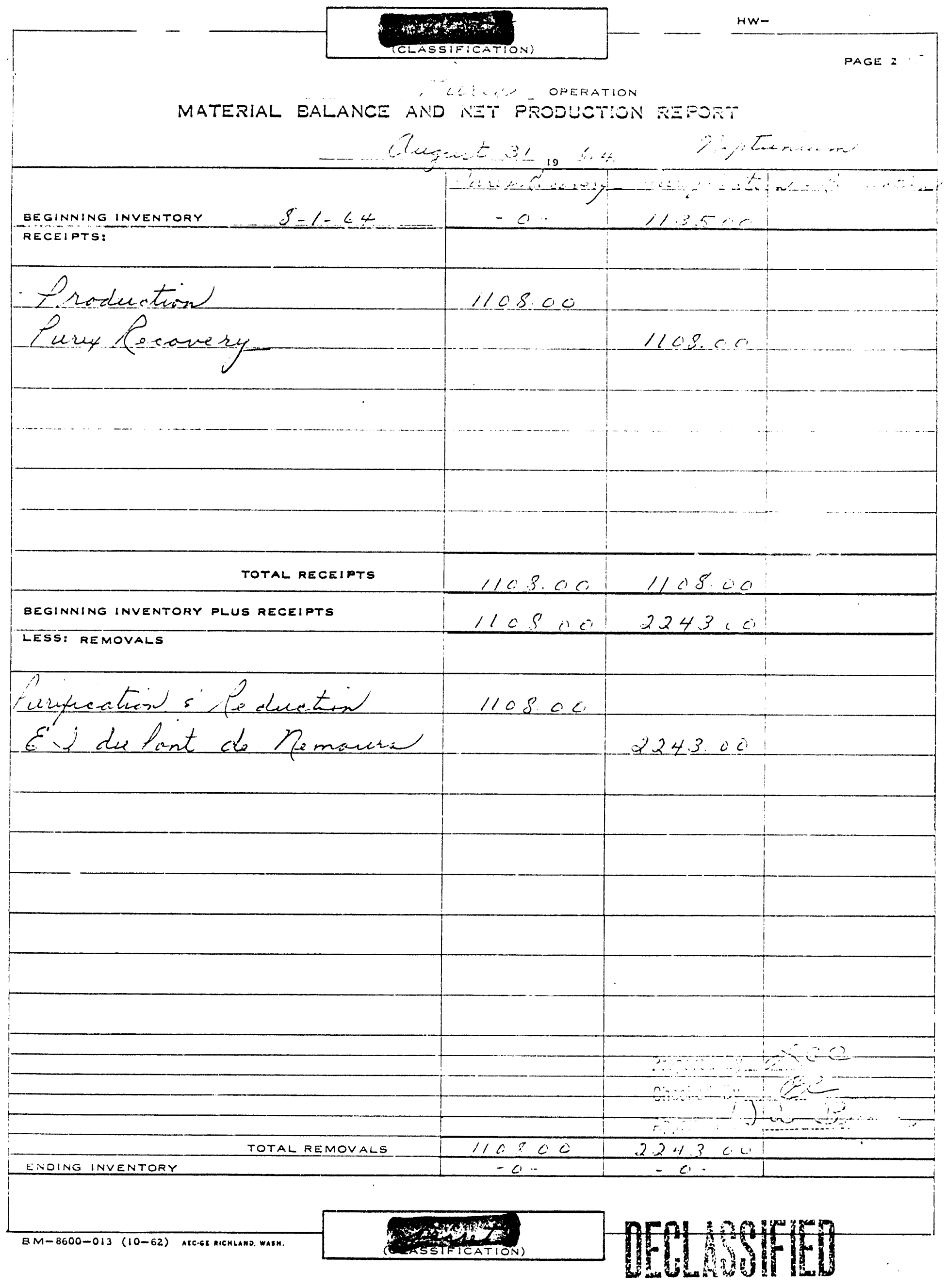




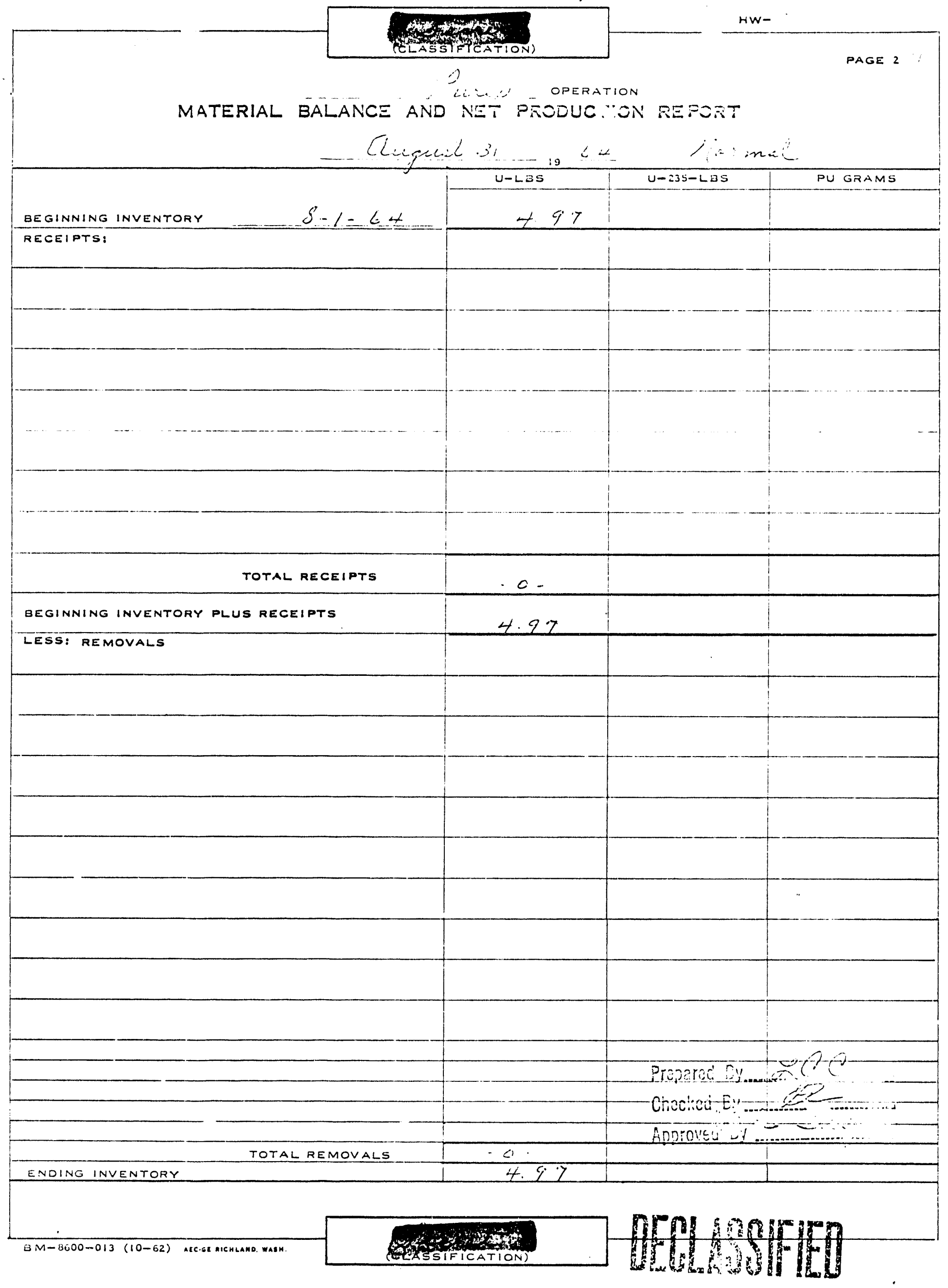




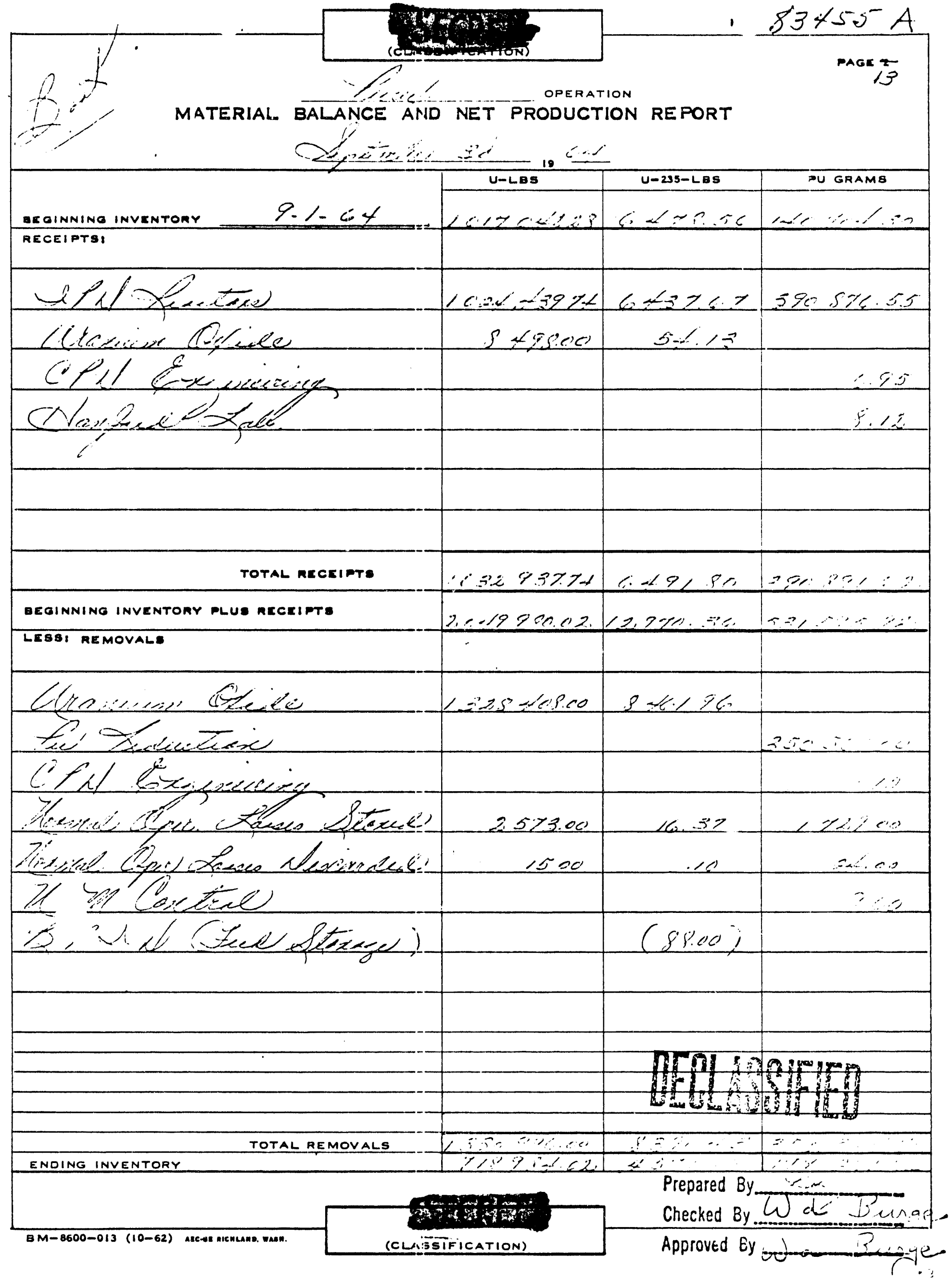




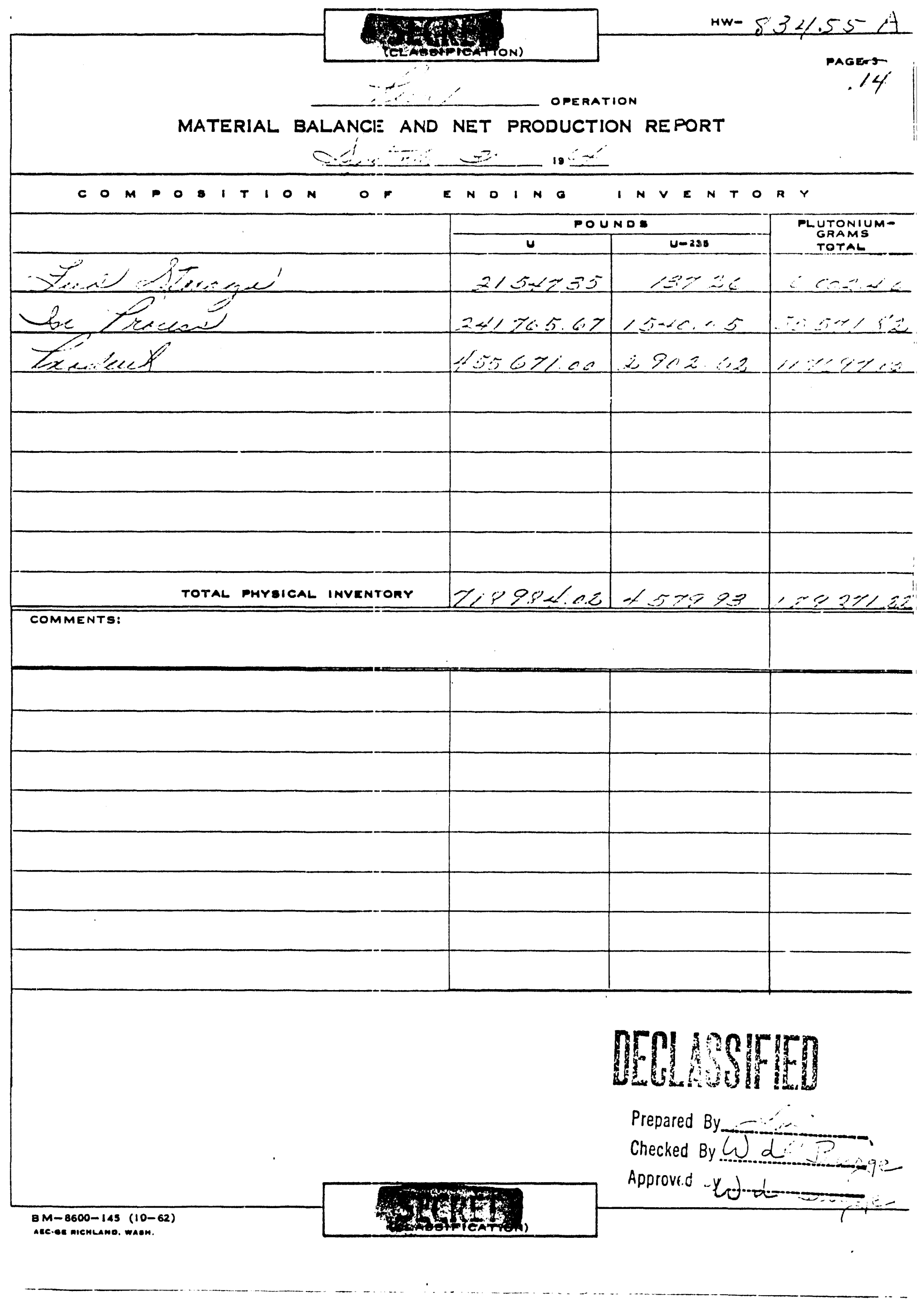




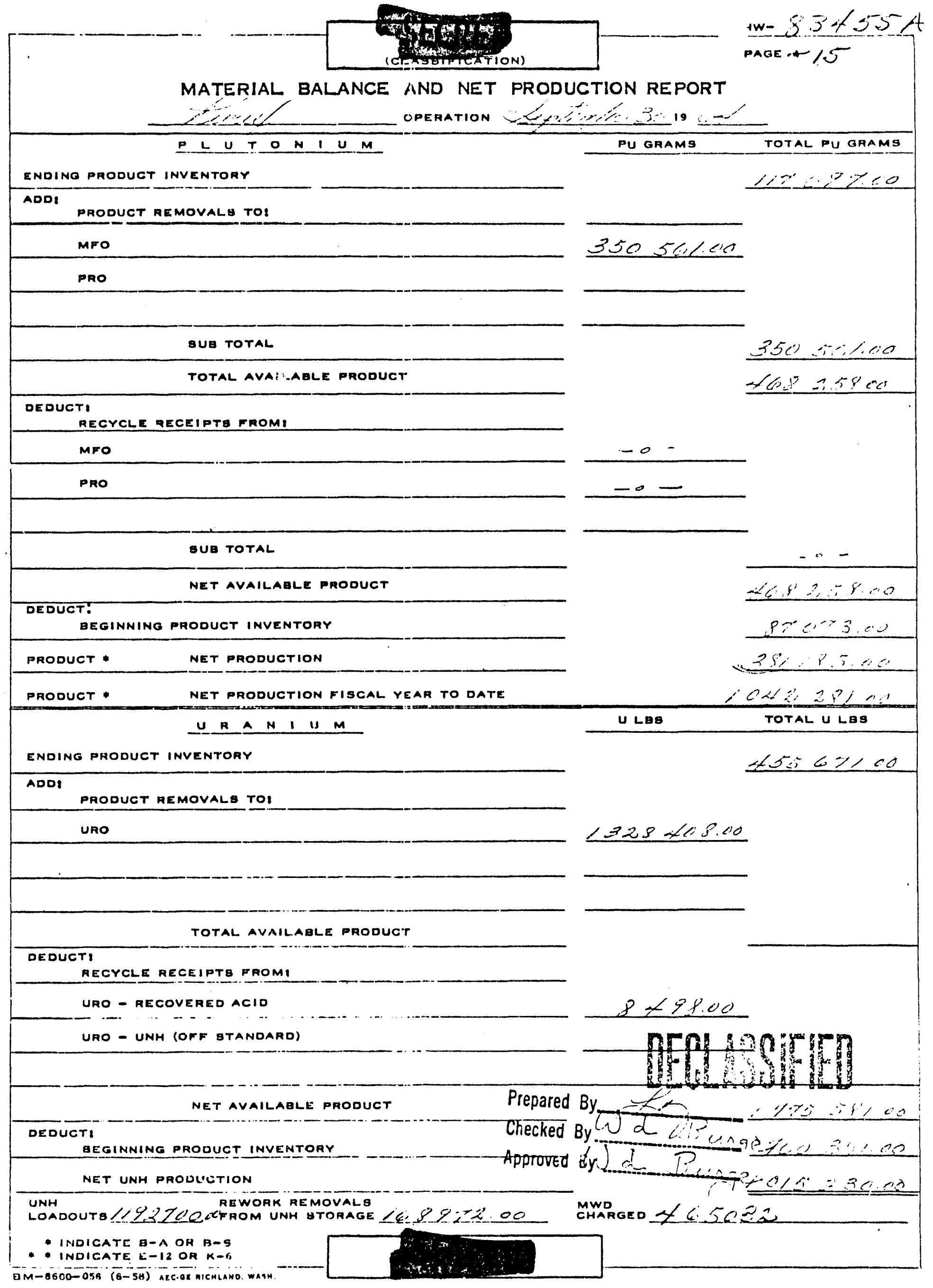




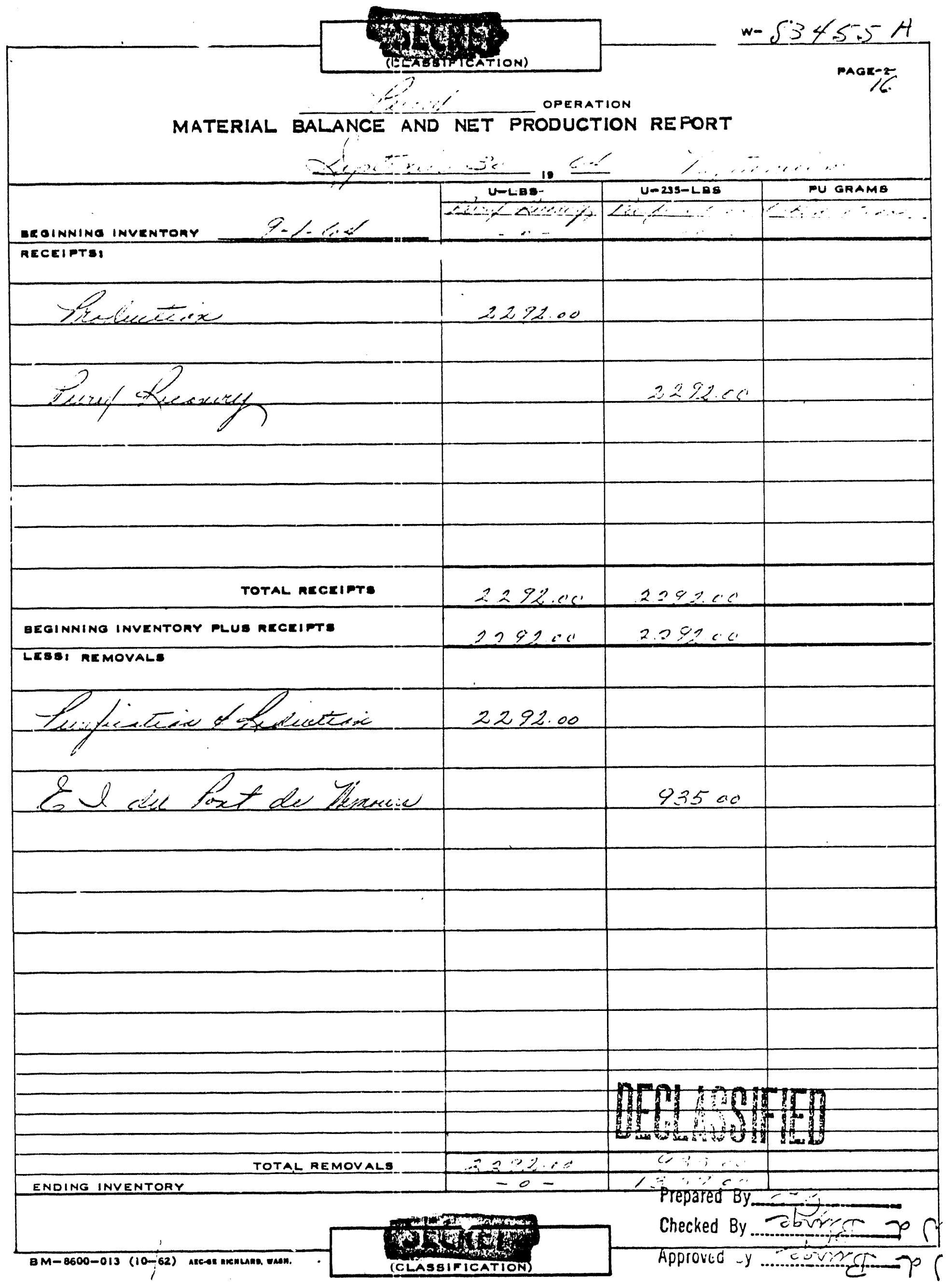




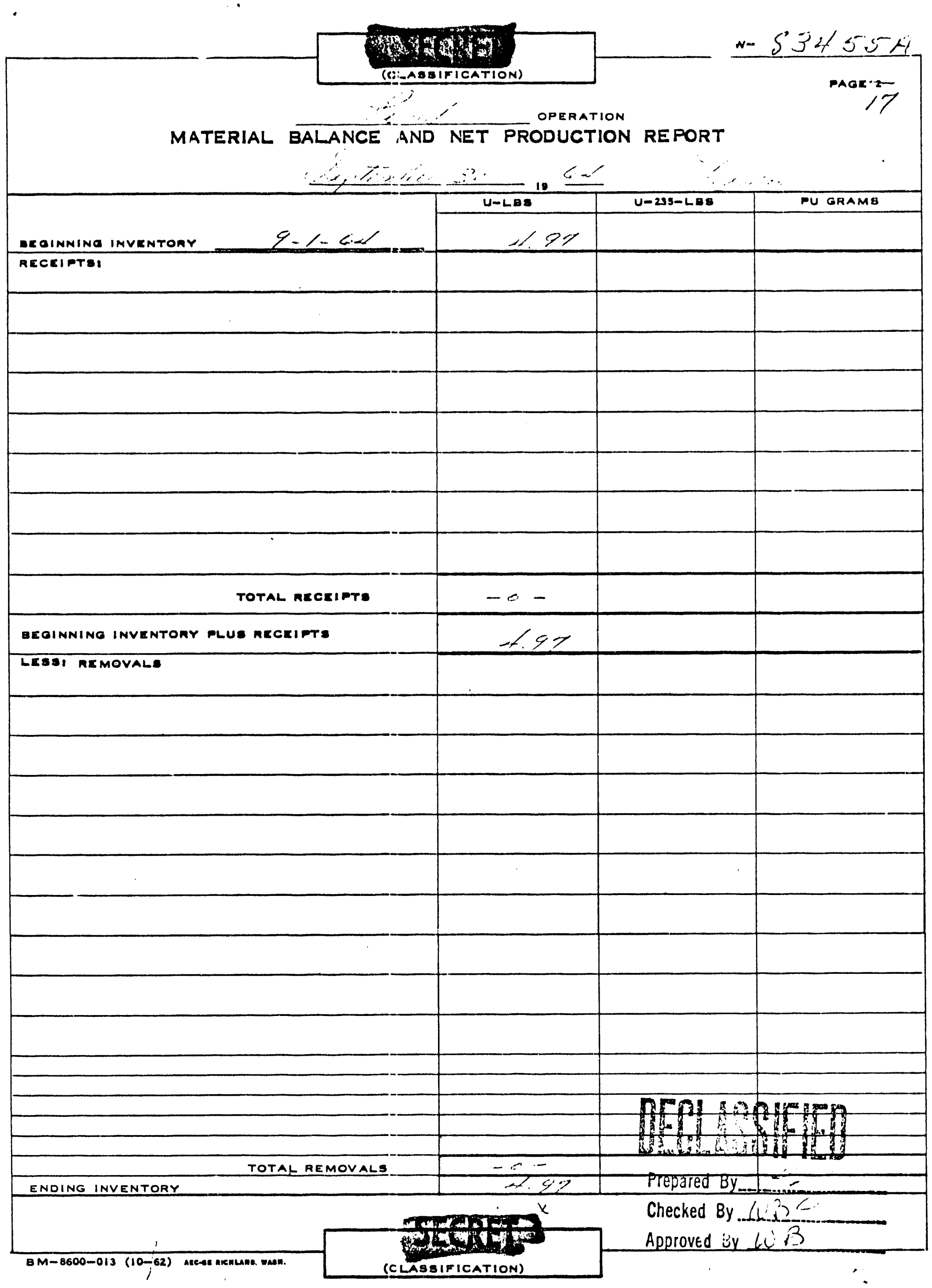




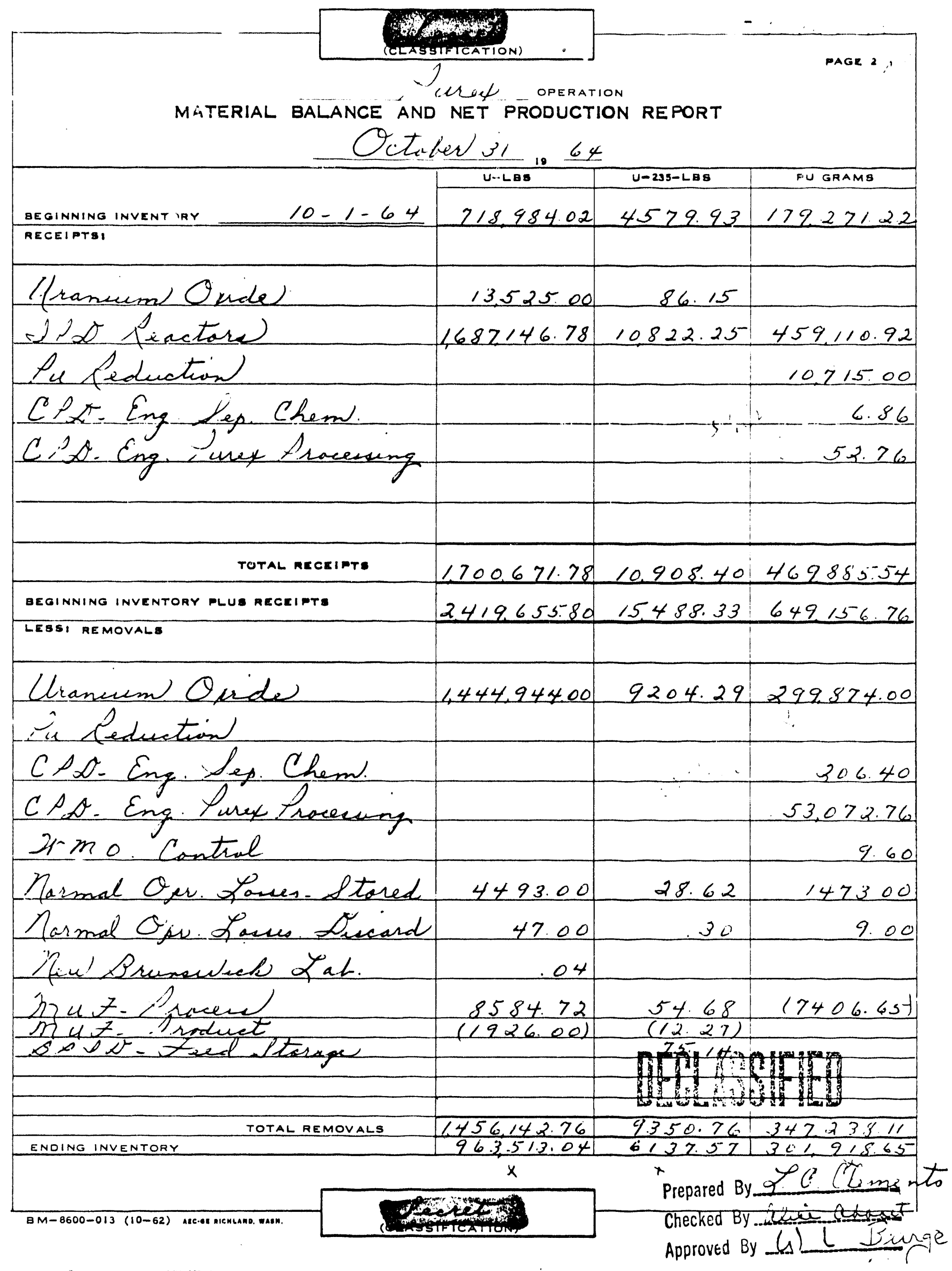




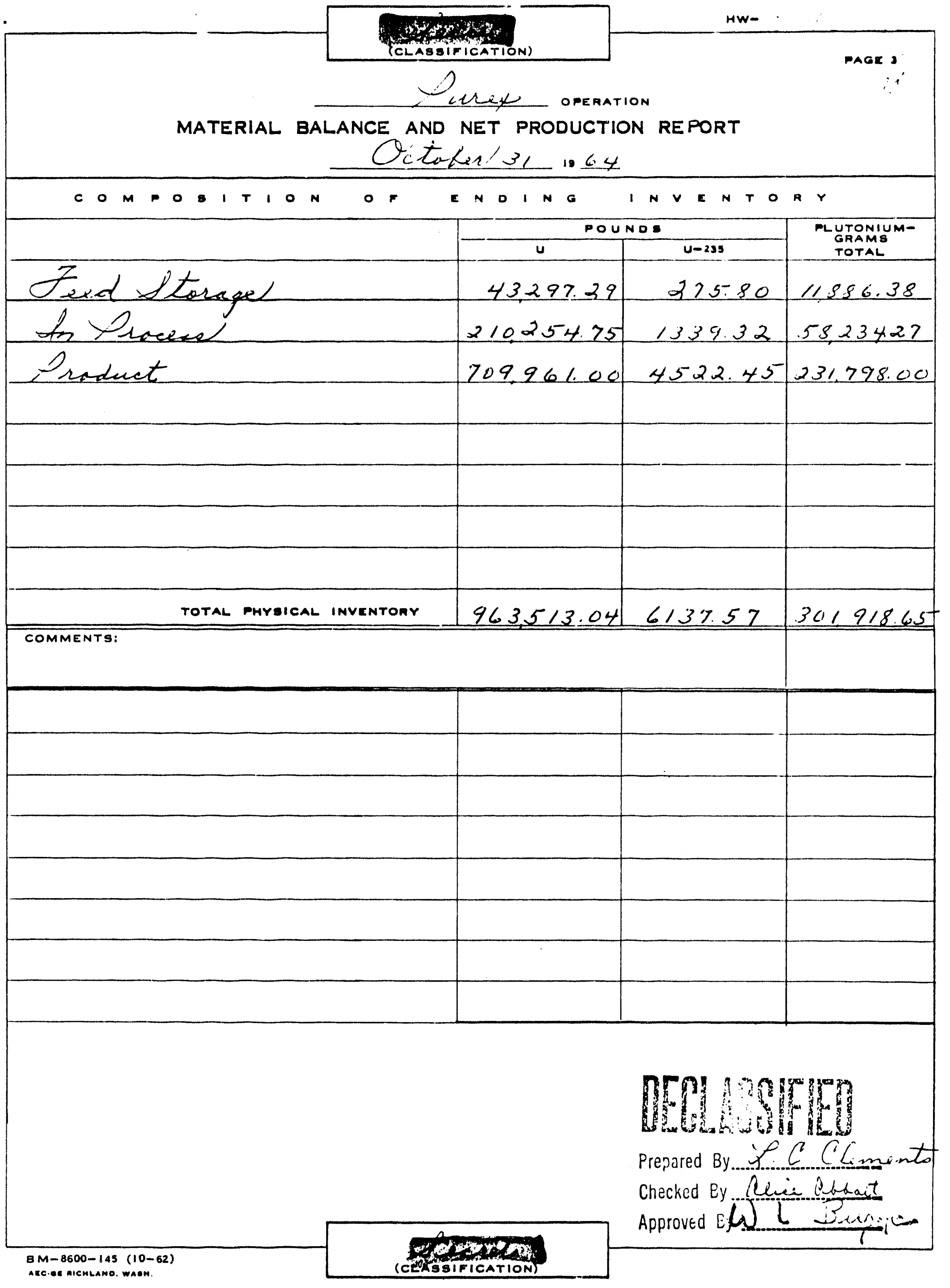




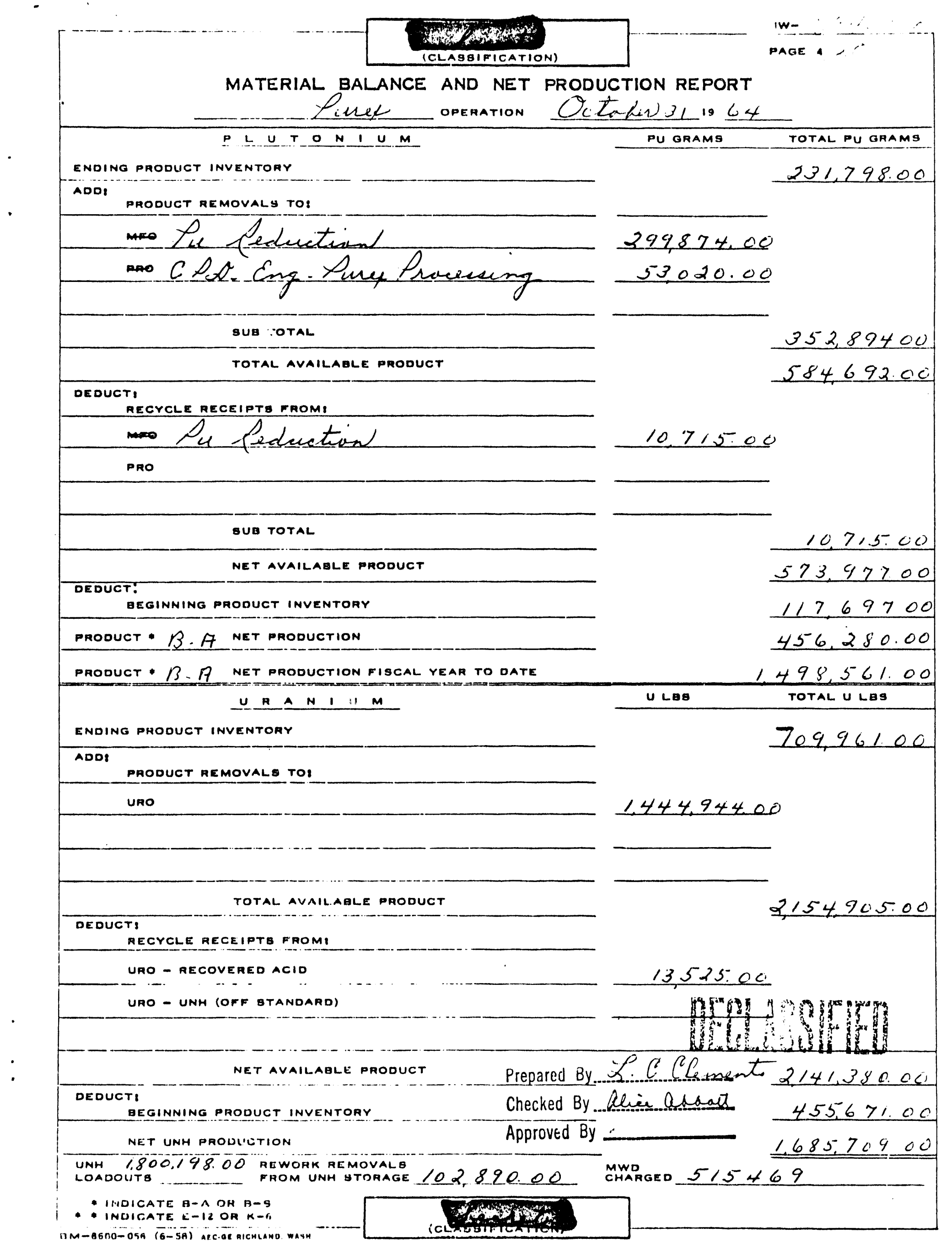




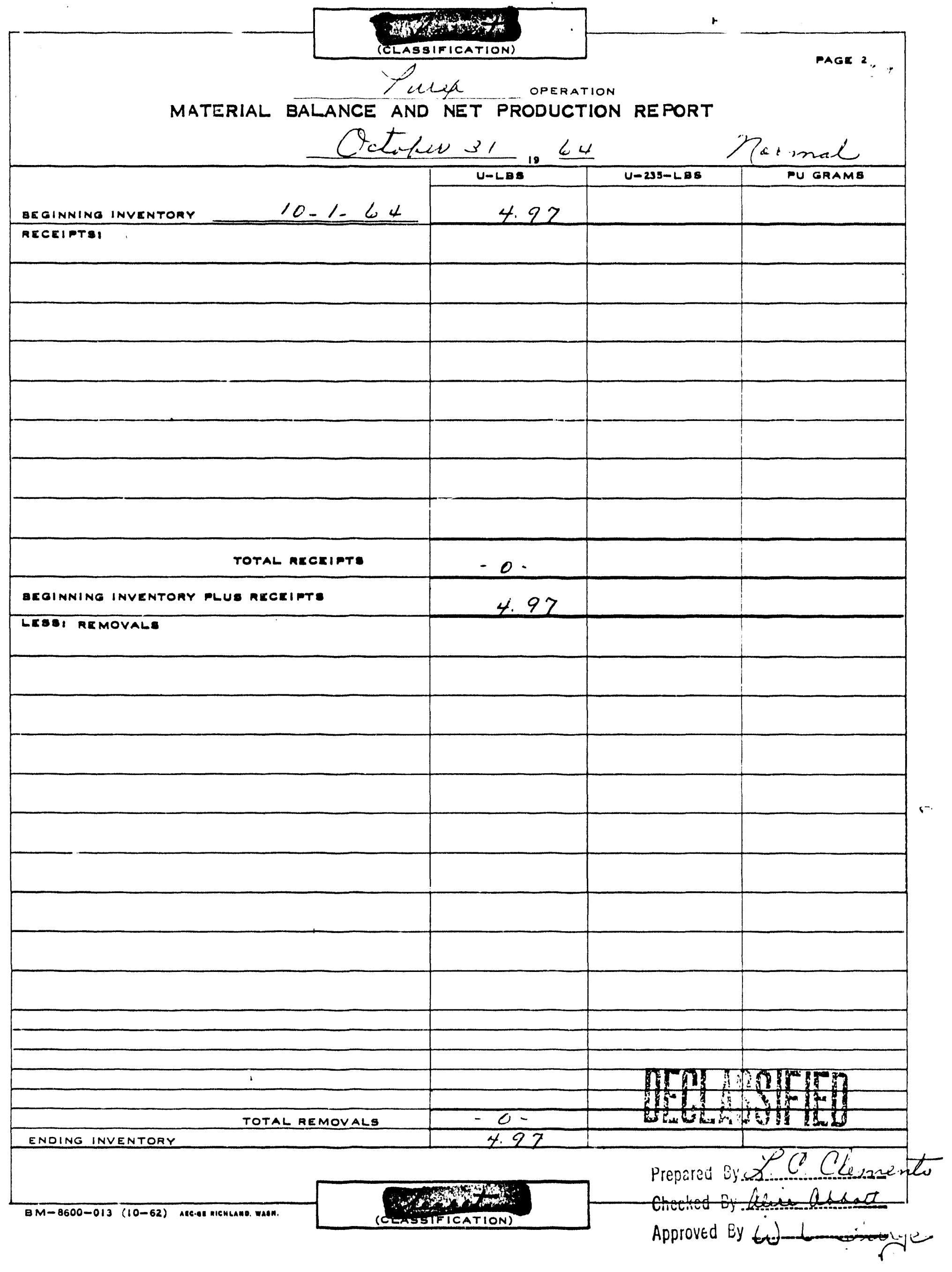




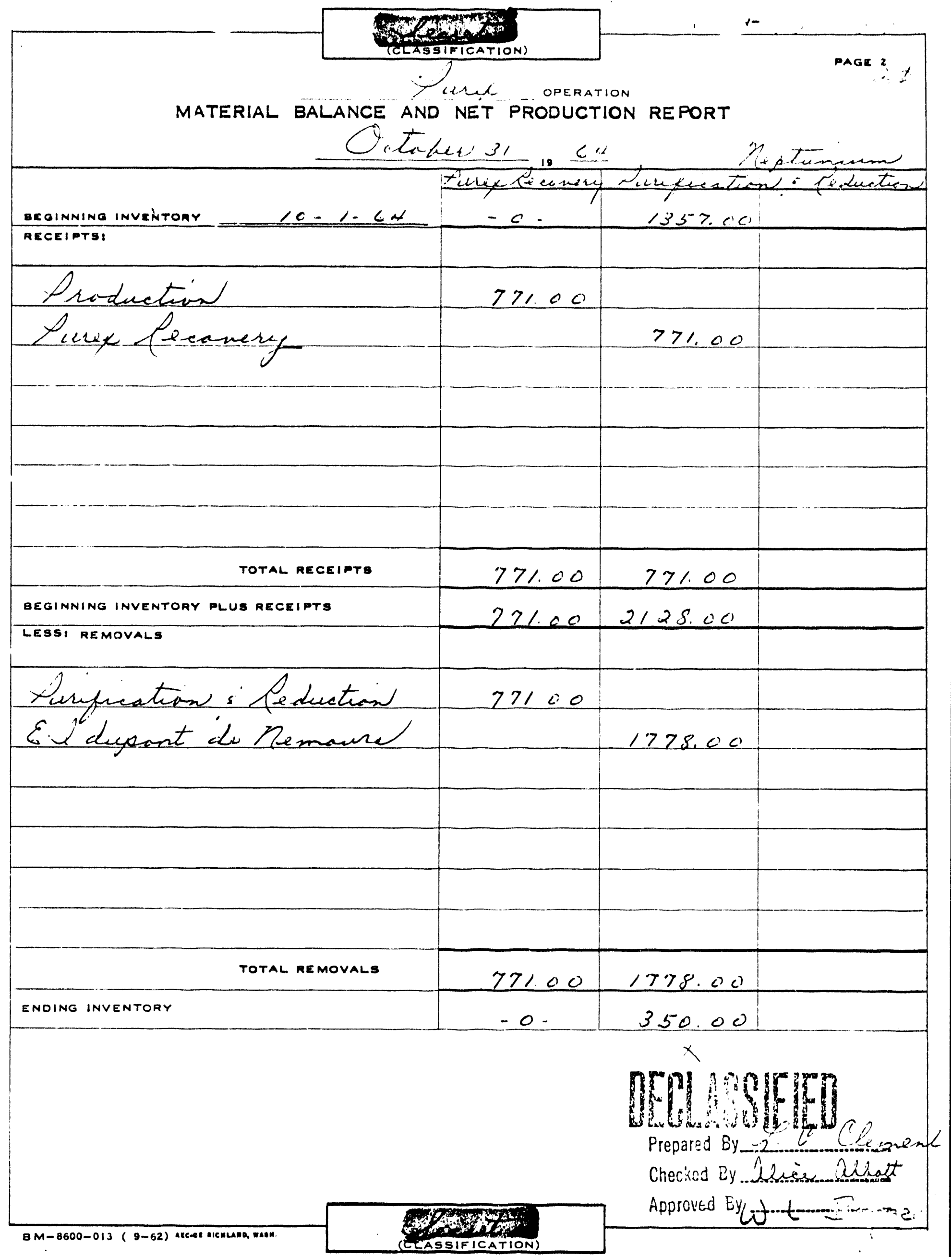




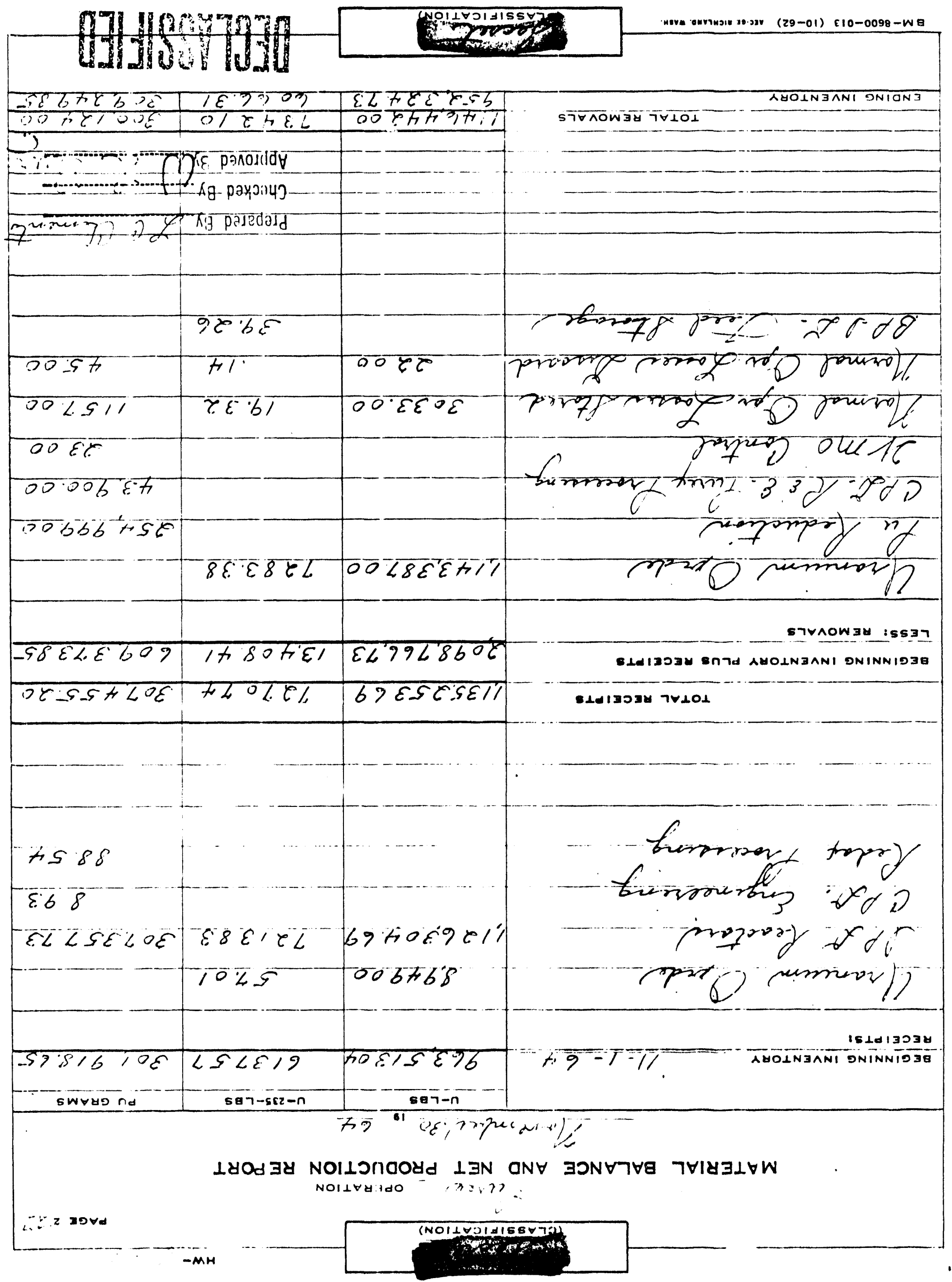




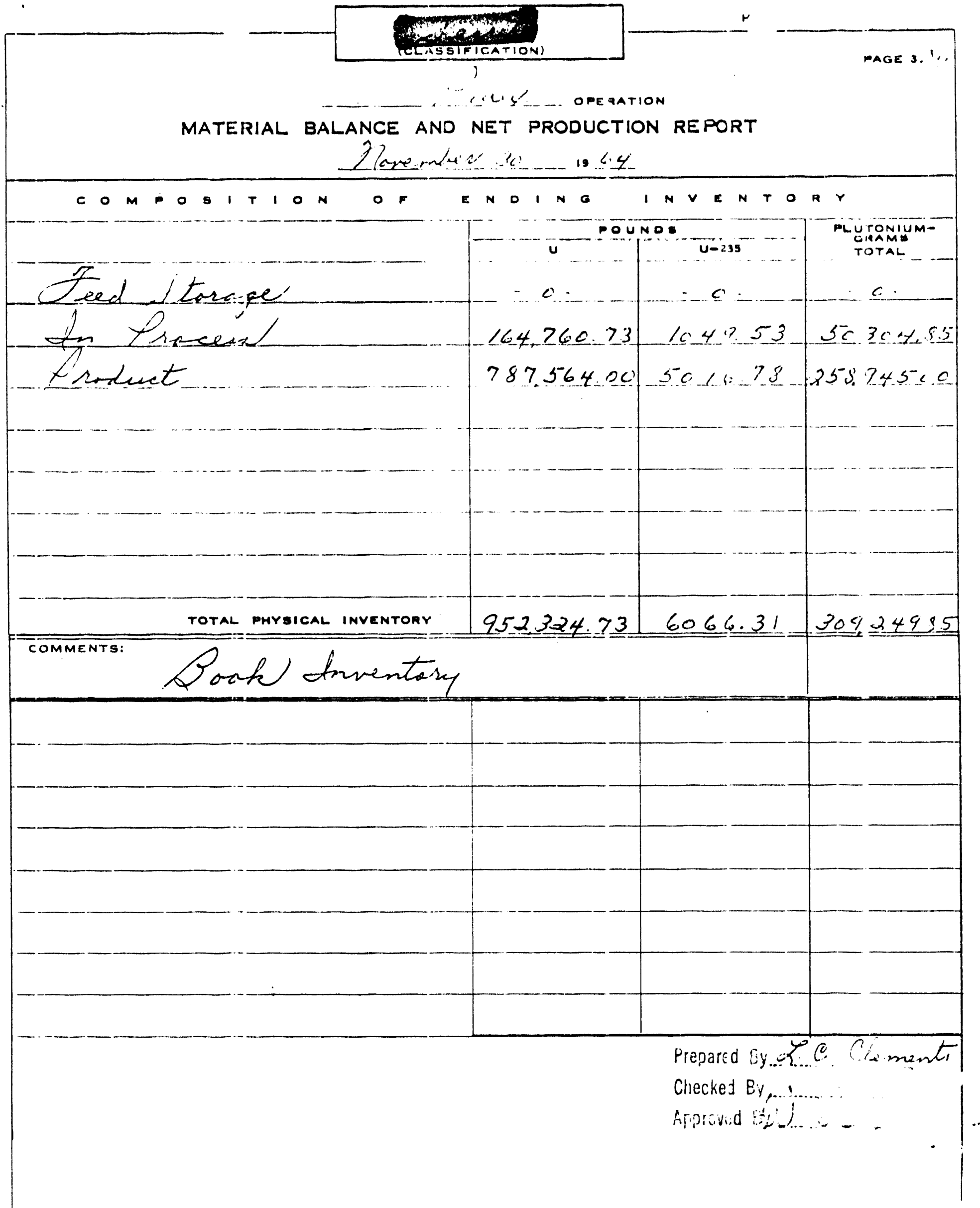

MATERIAL BALANCE AND NET PRODUCTION REPORT

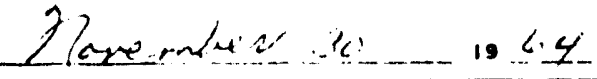




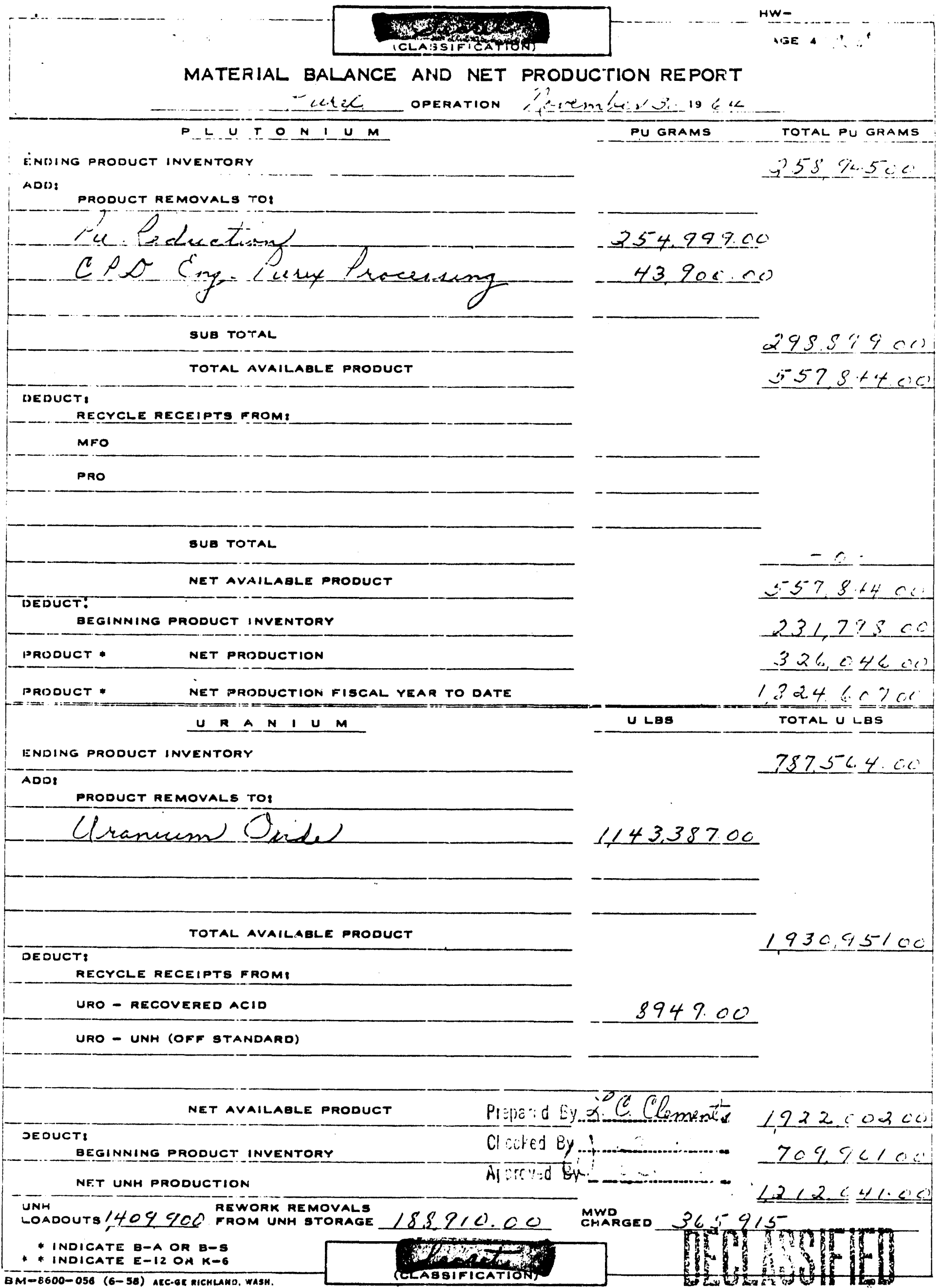




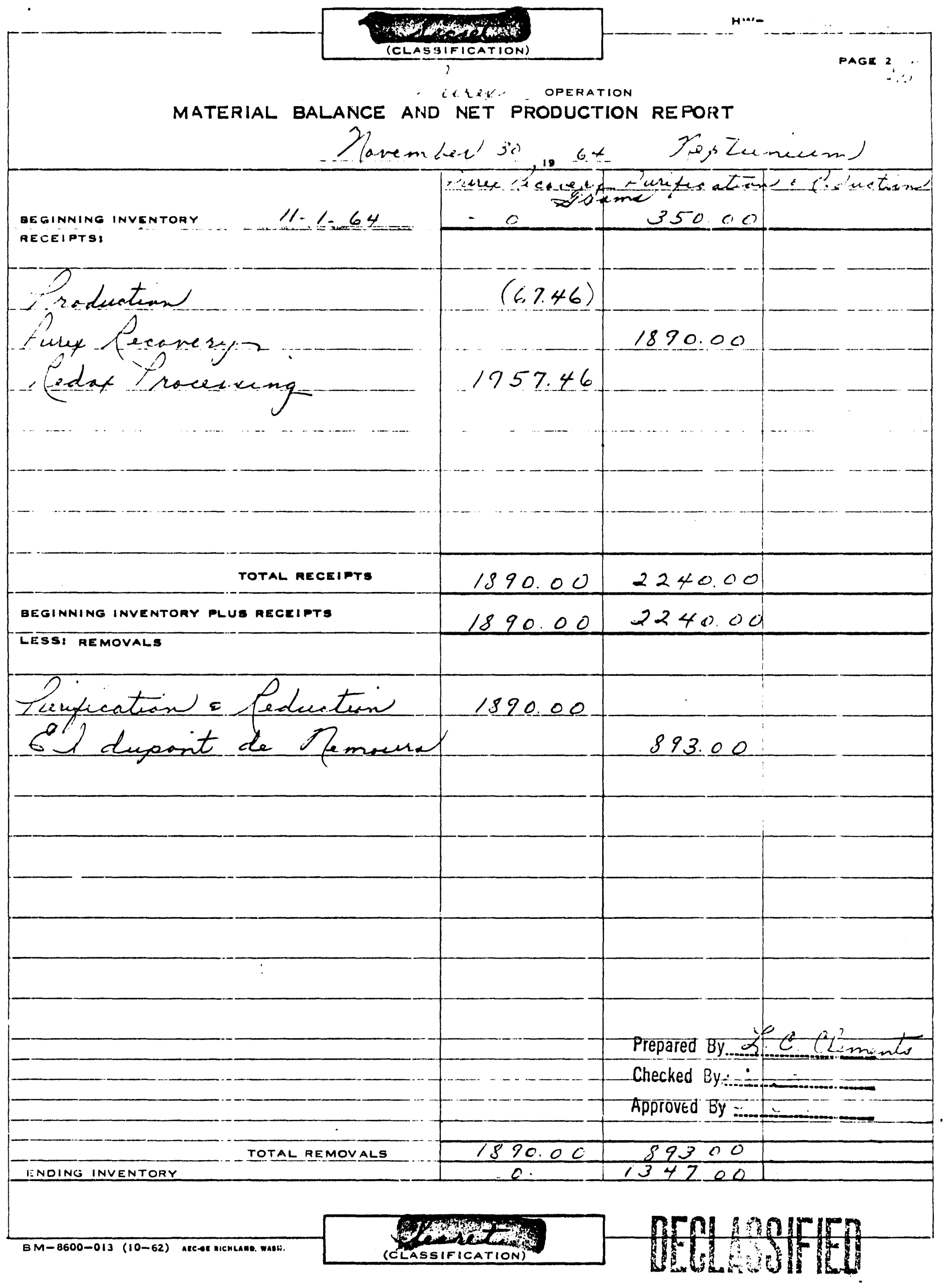




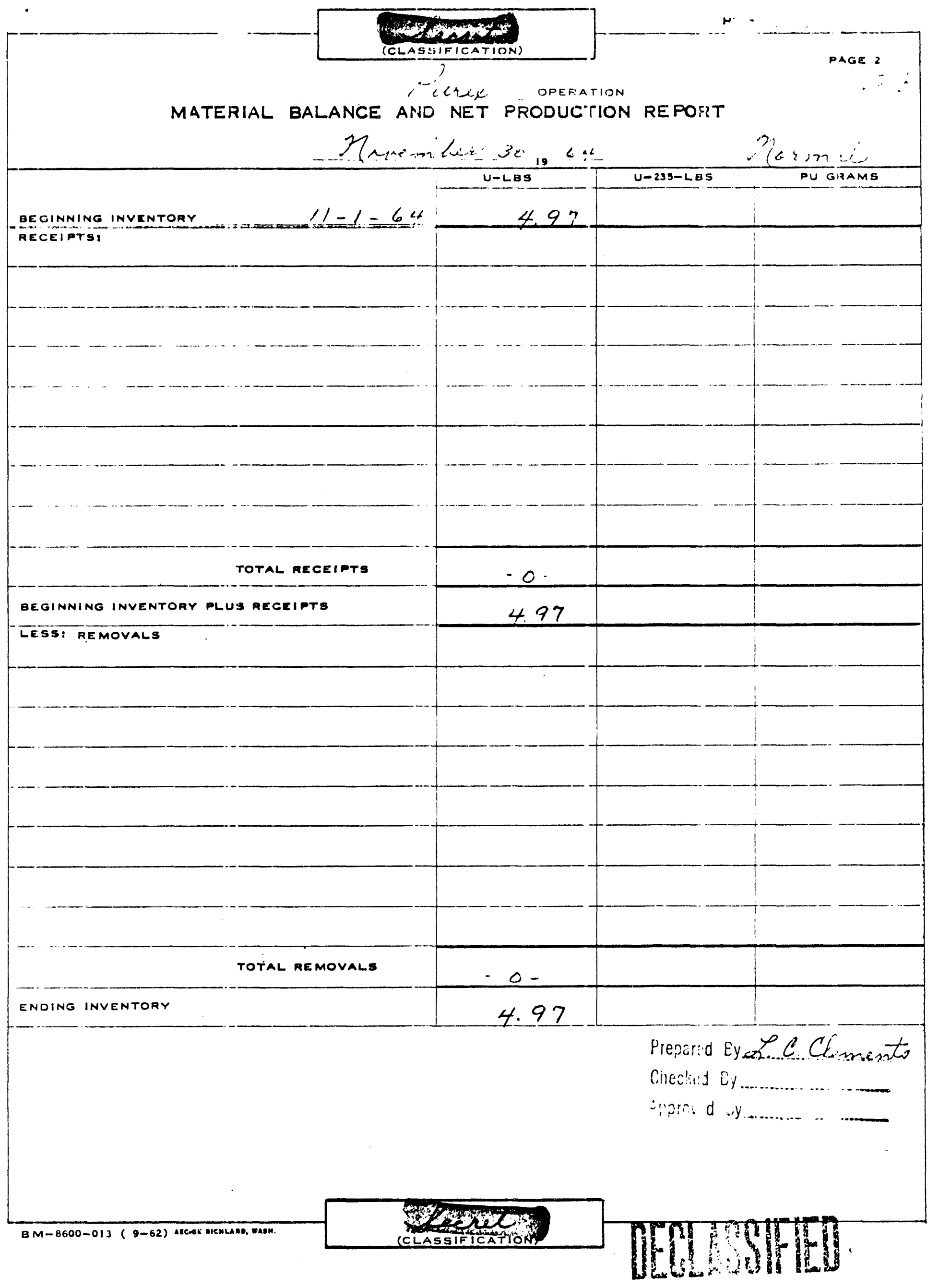




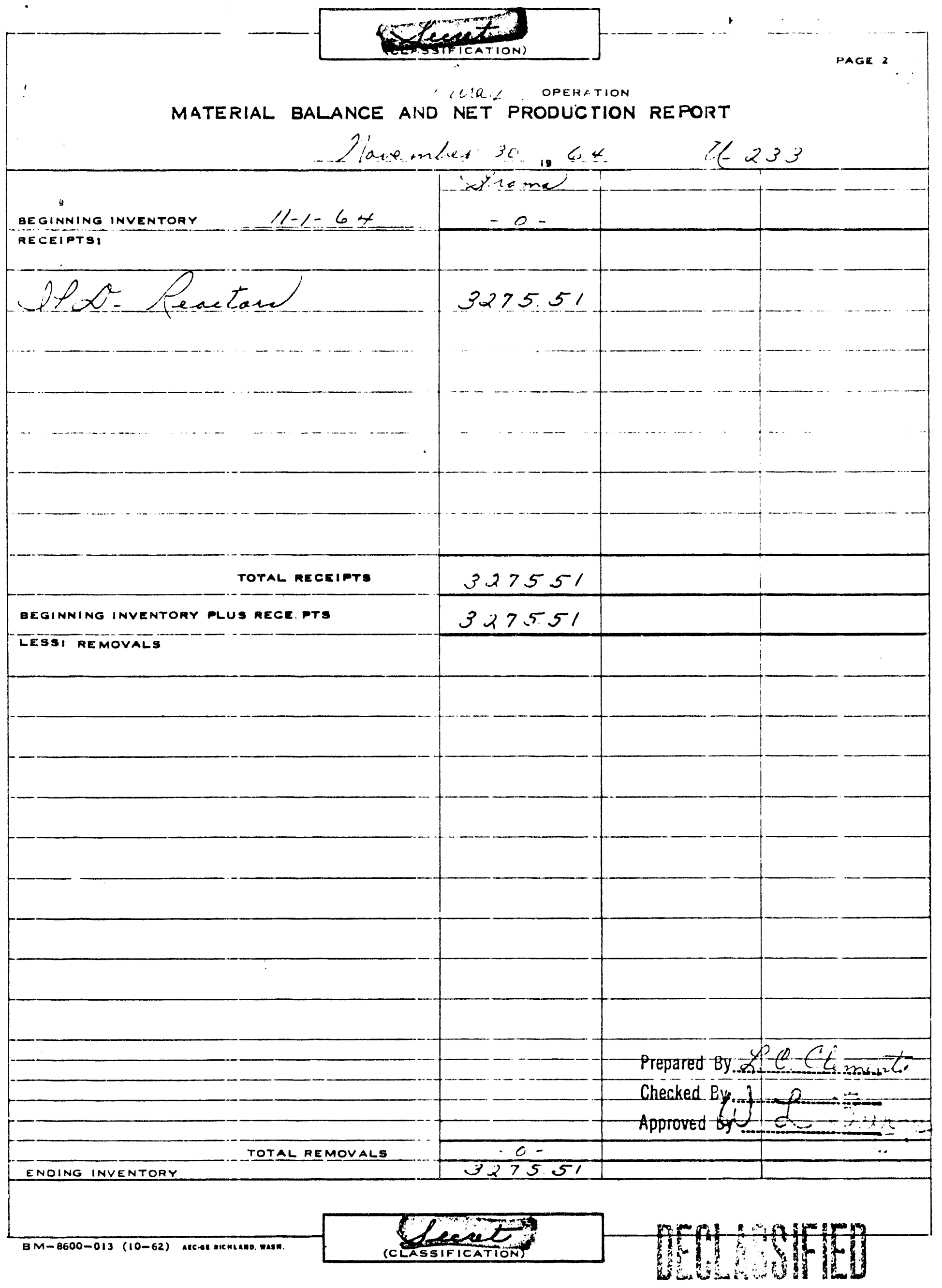




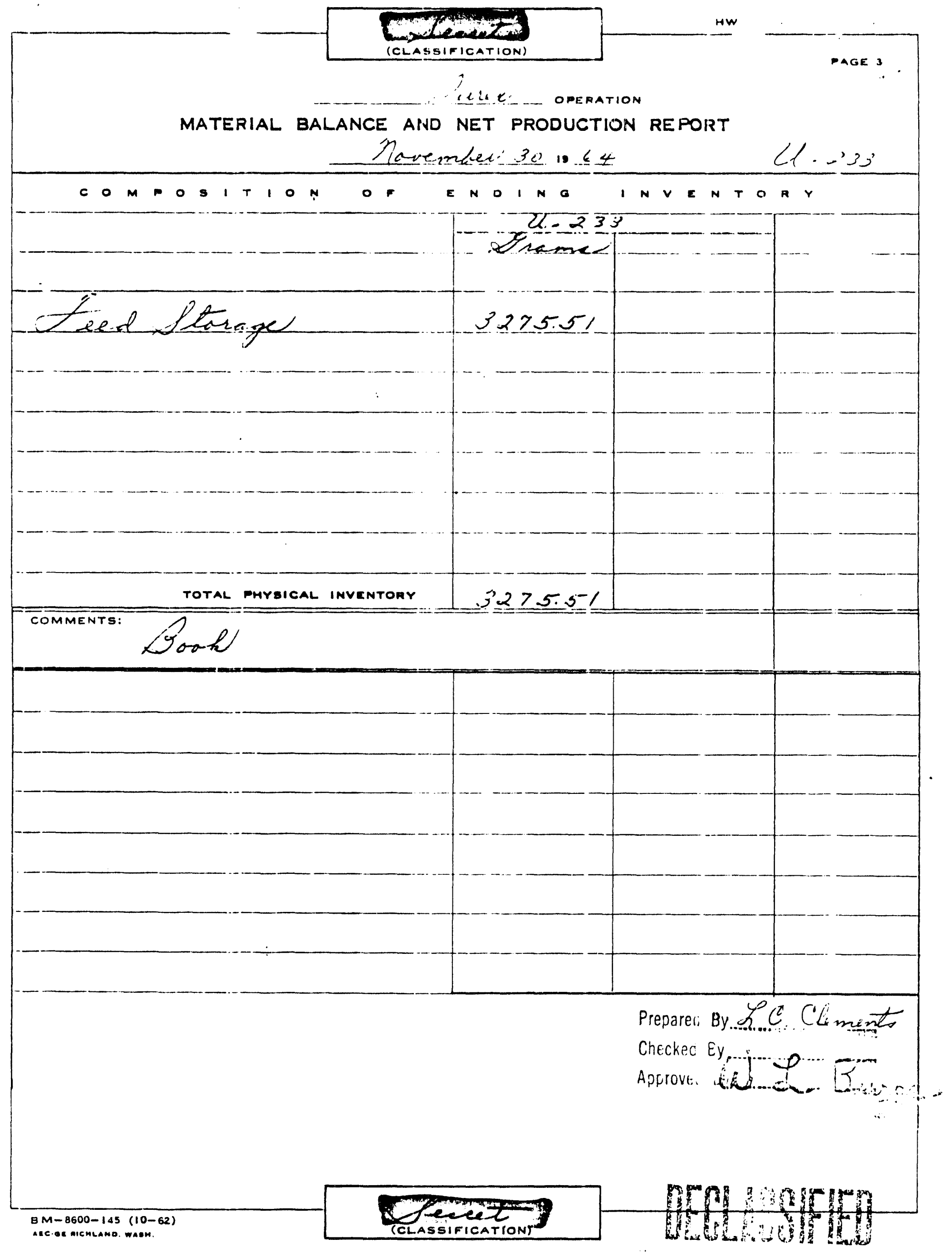




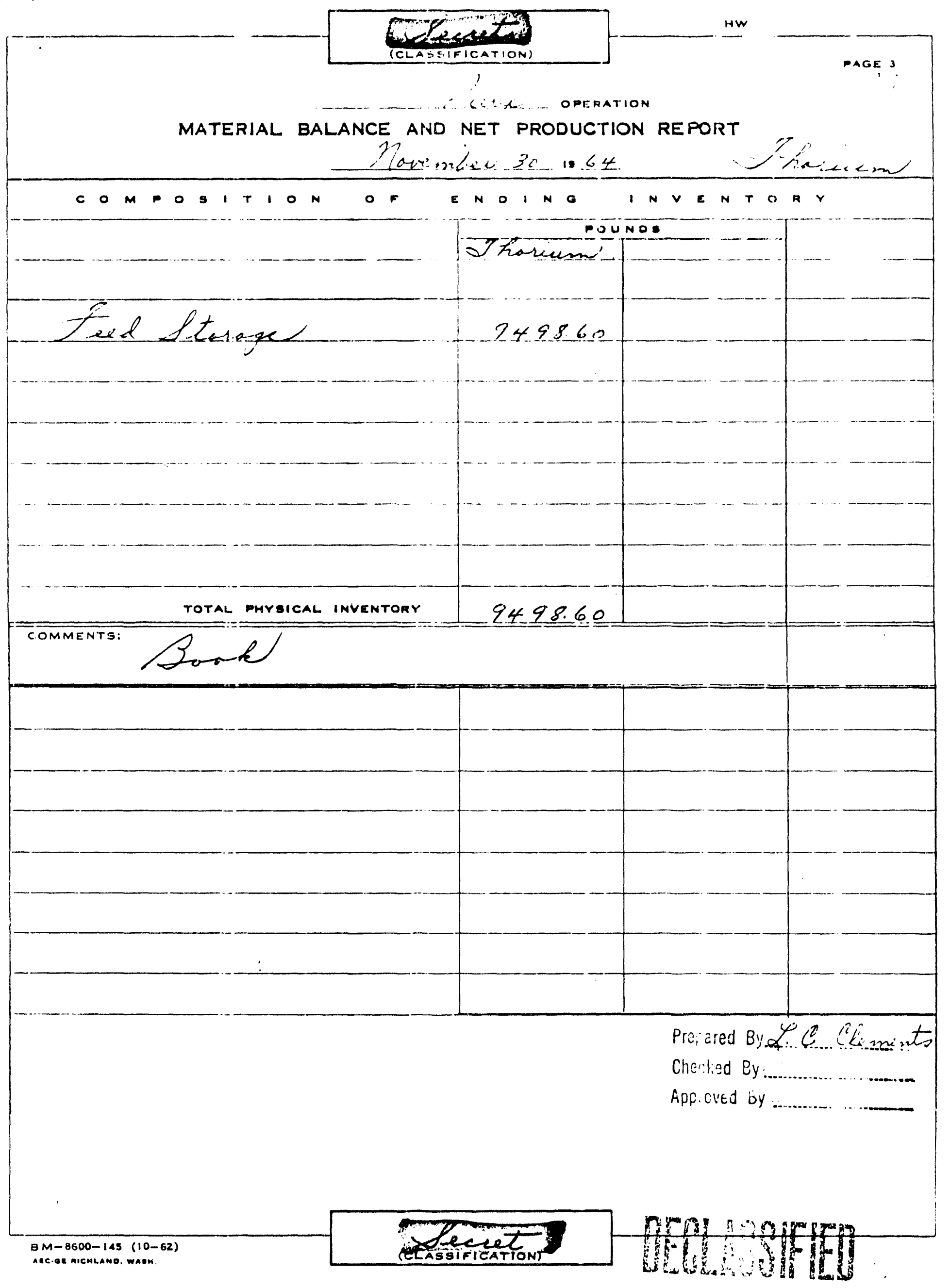




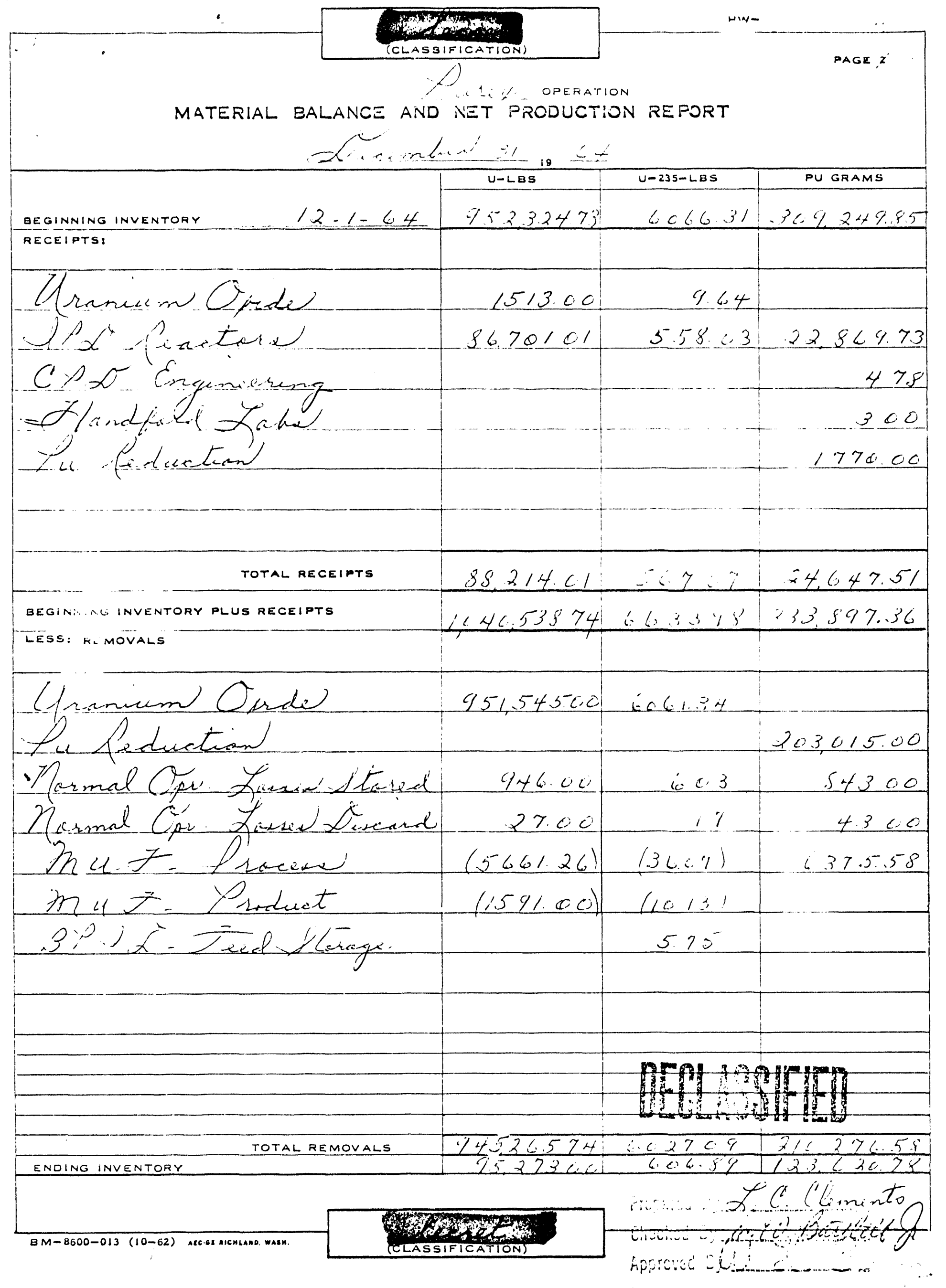




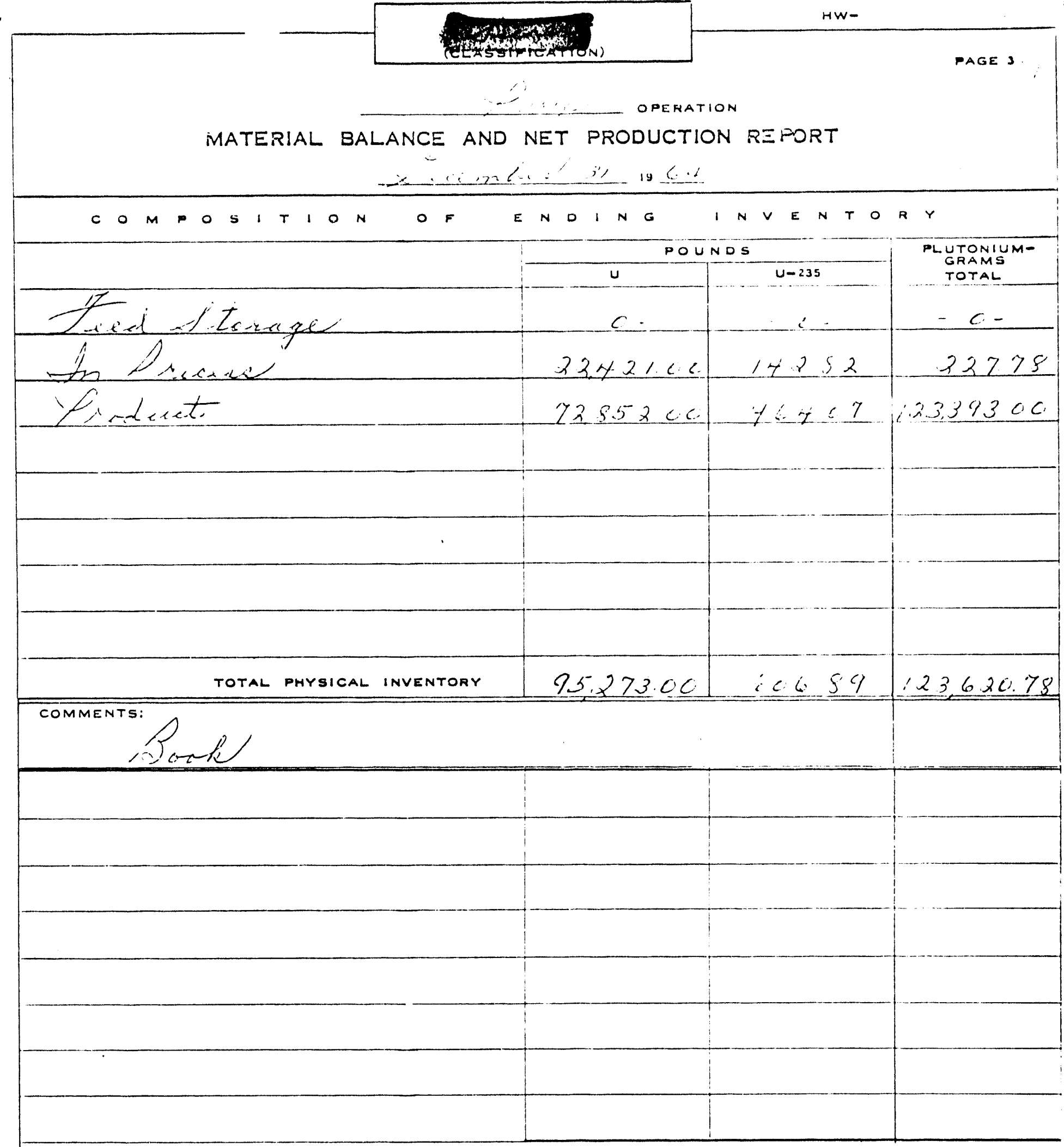




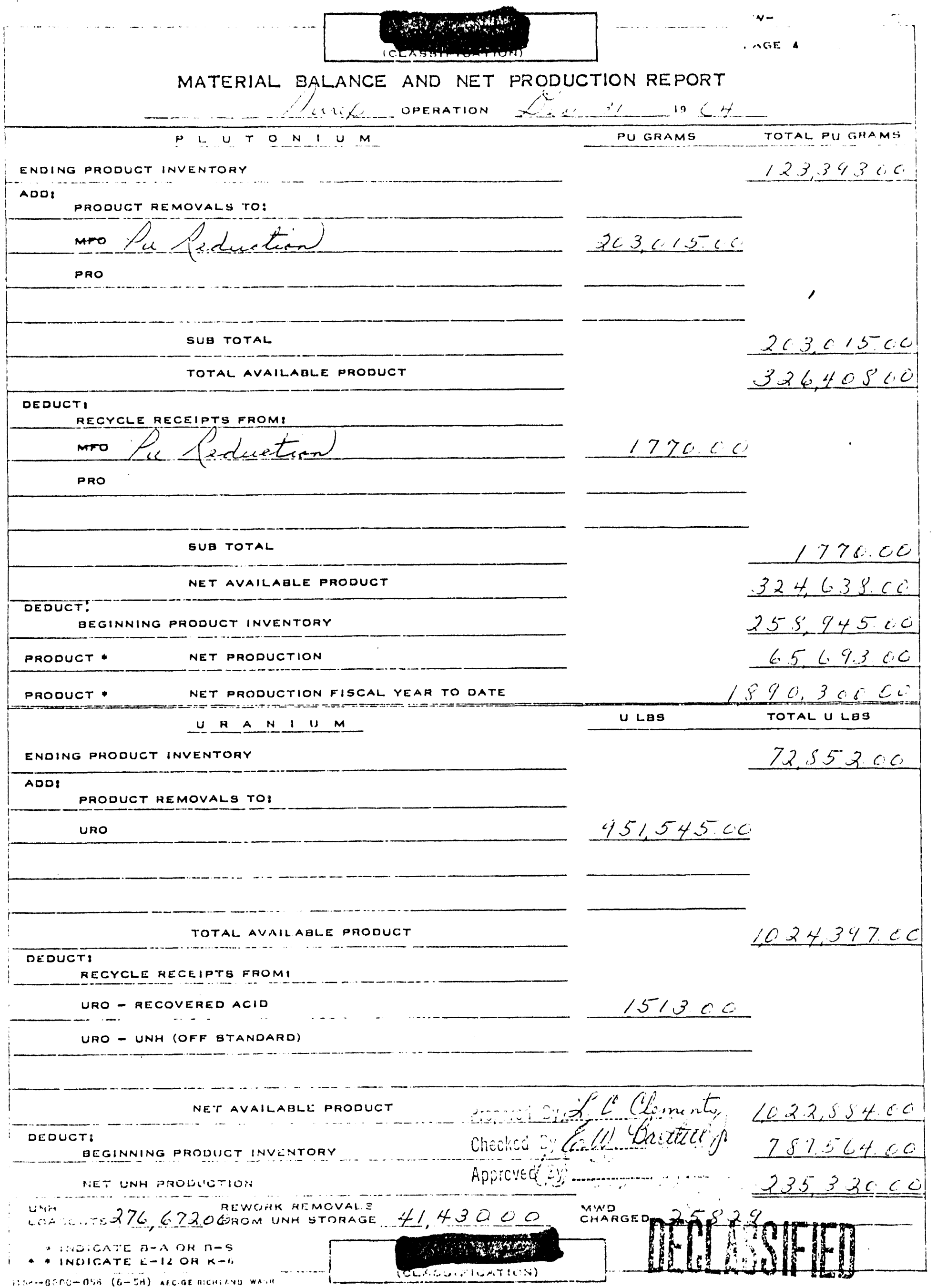




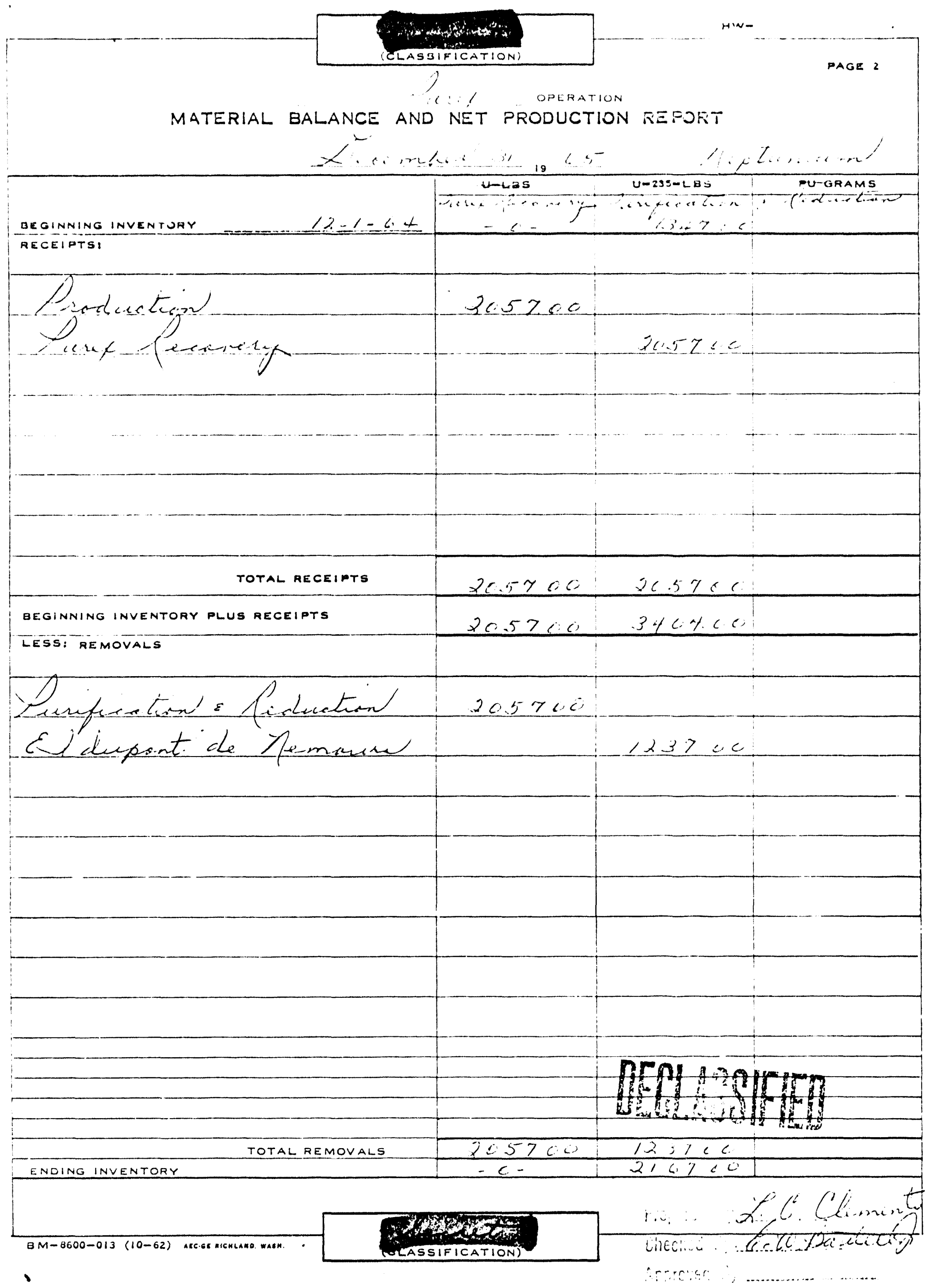




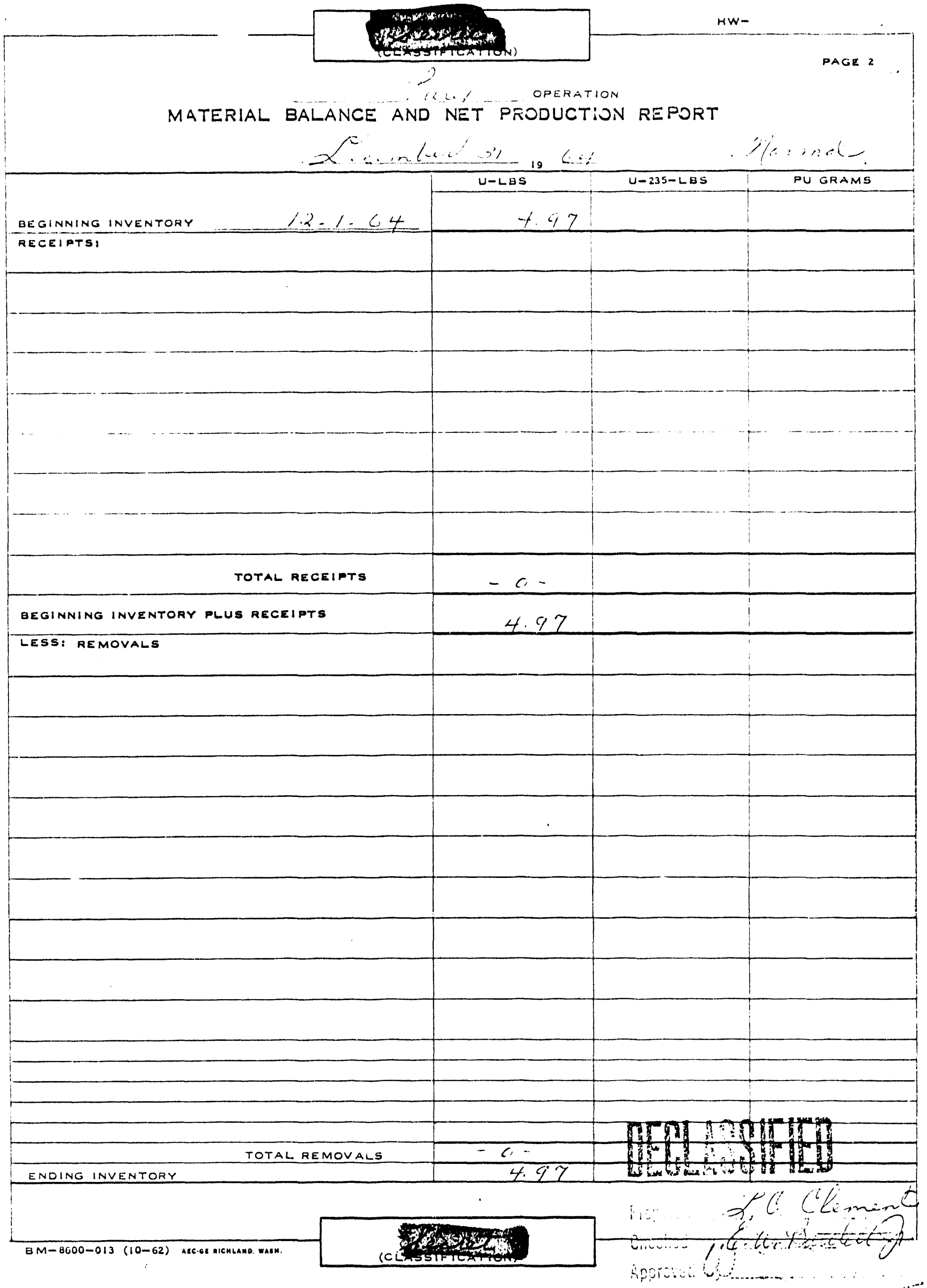




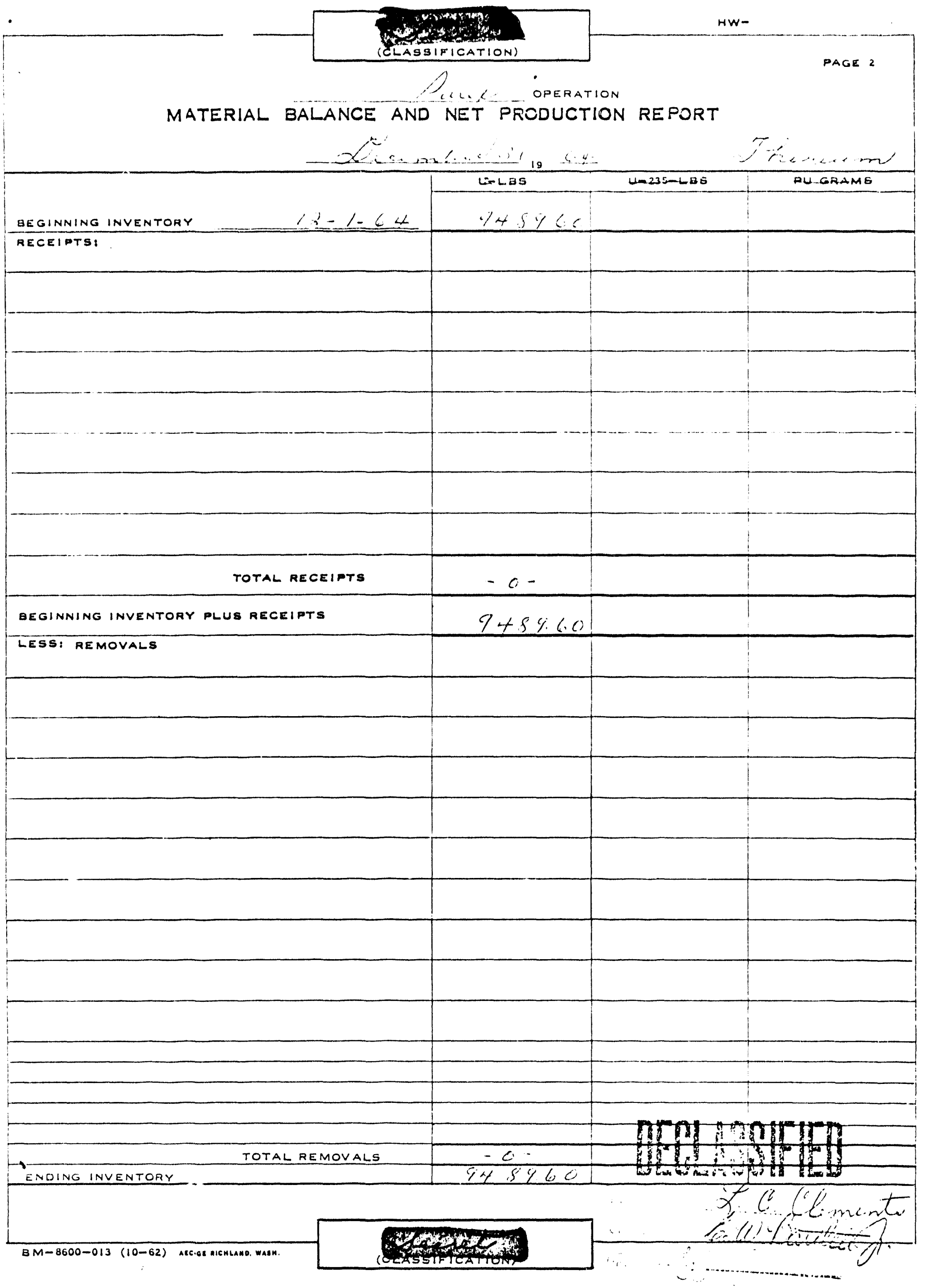




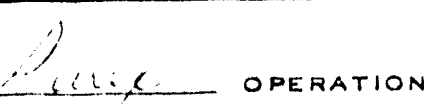

MATERIAL BALANCE AND NET PRODUCTION REFORT

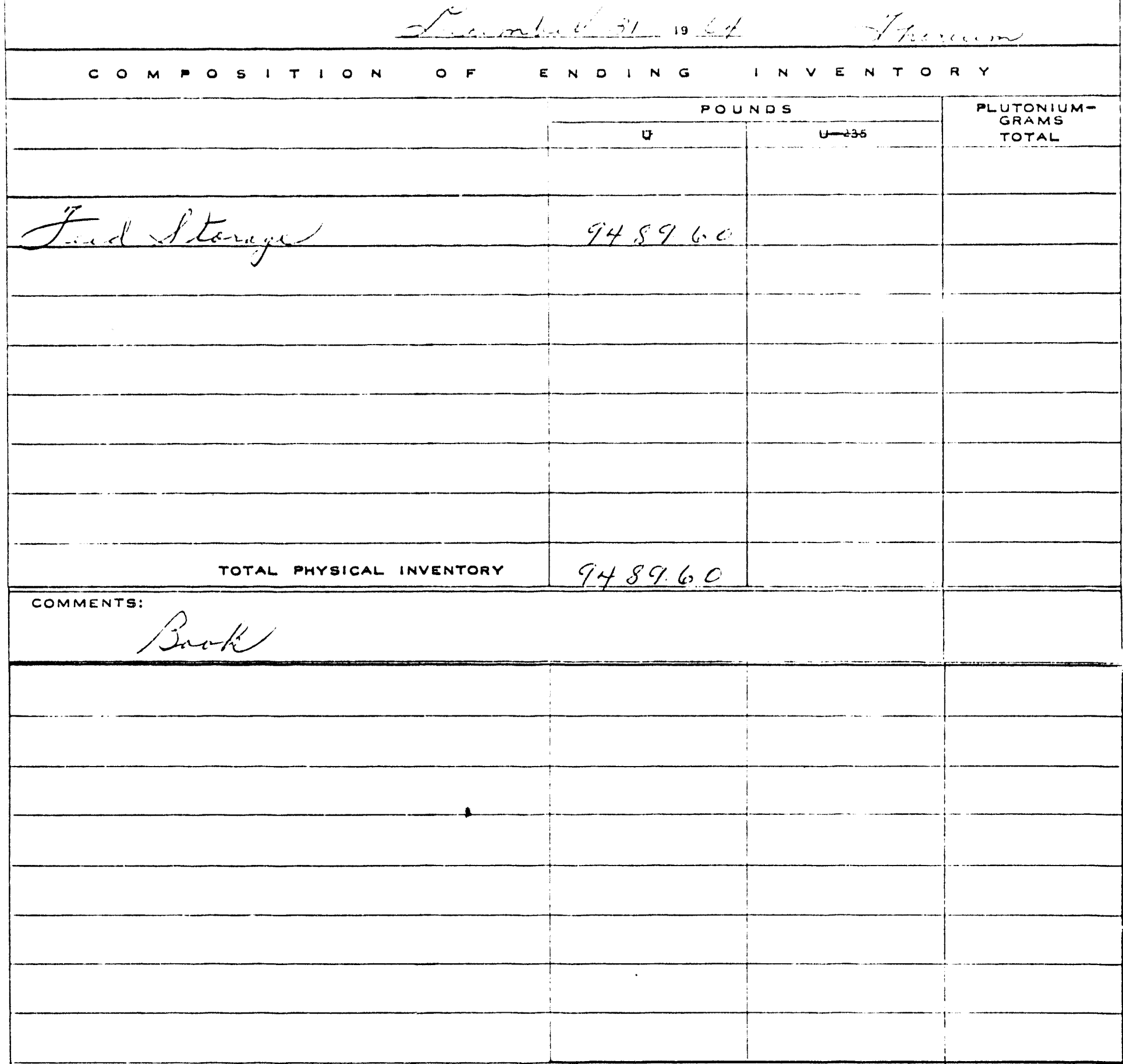




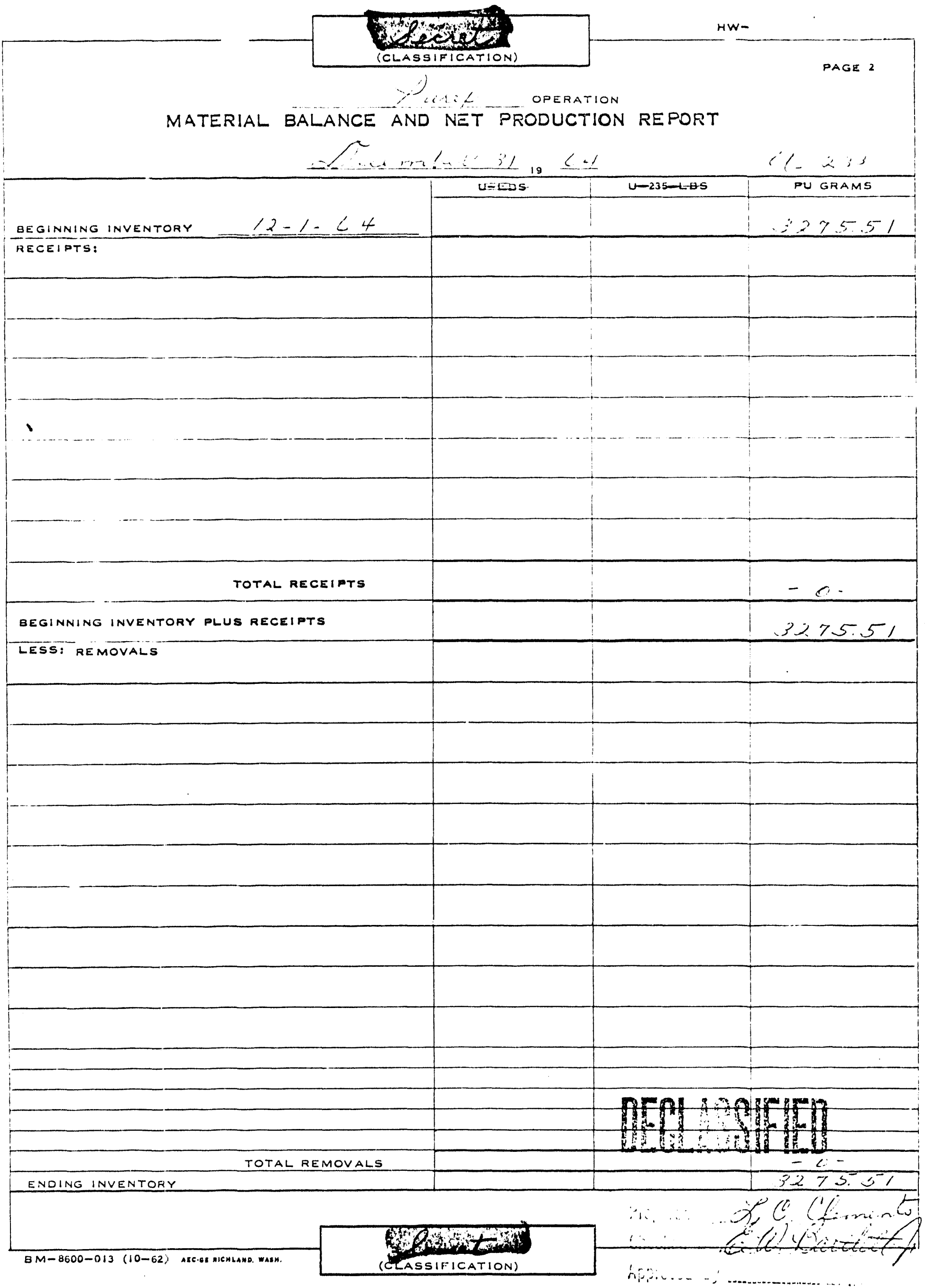




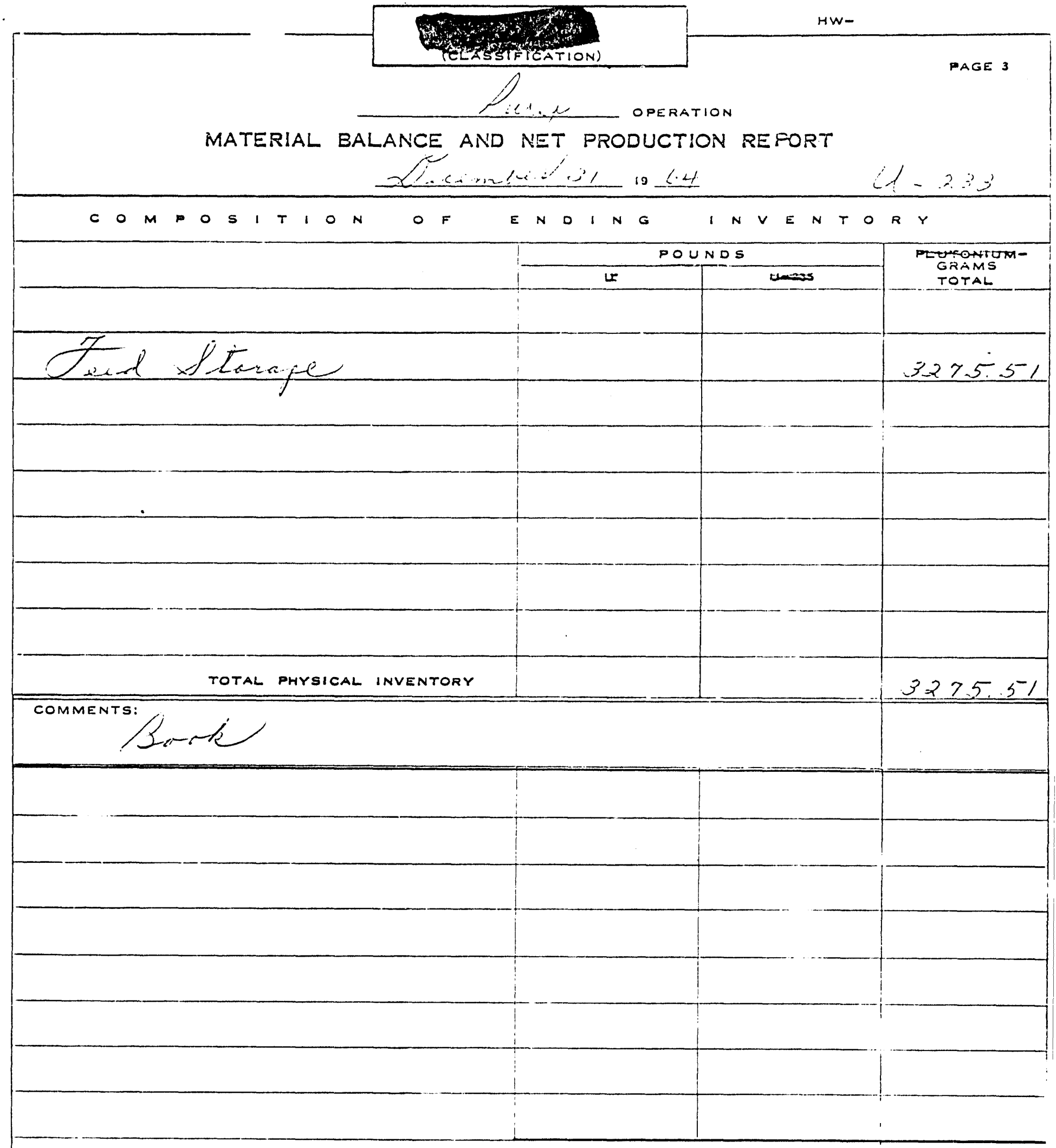




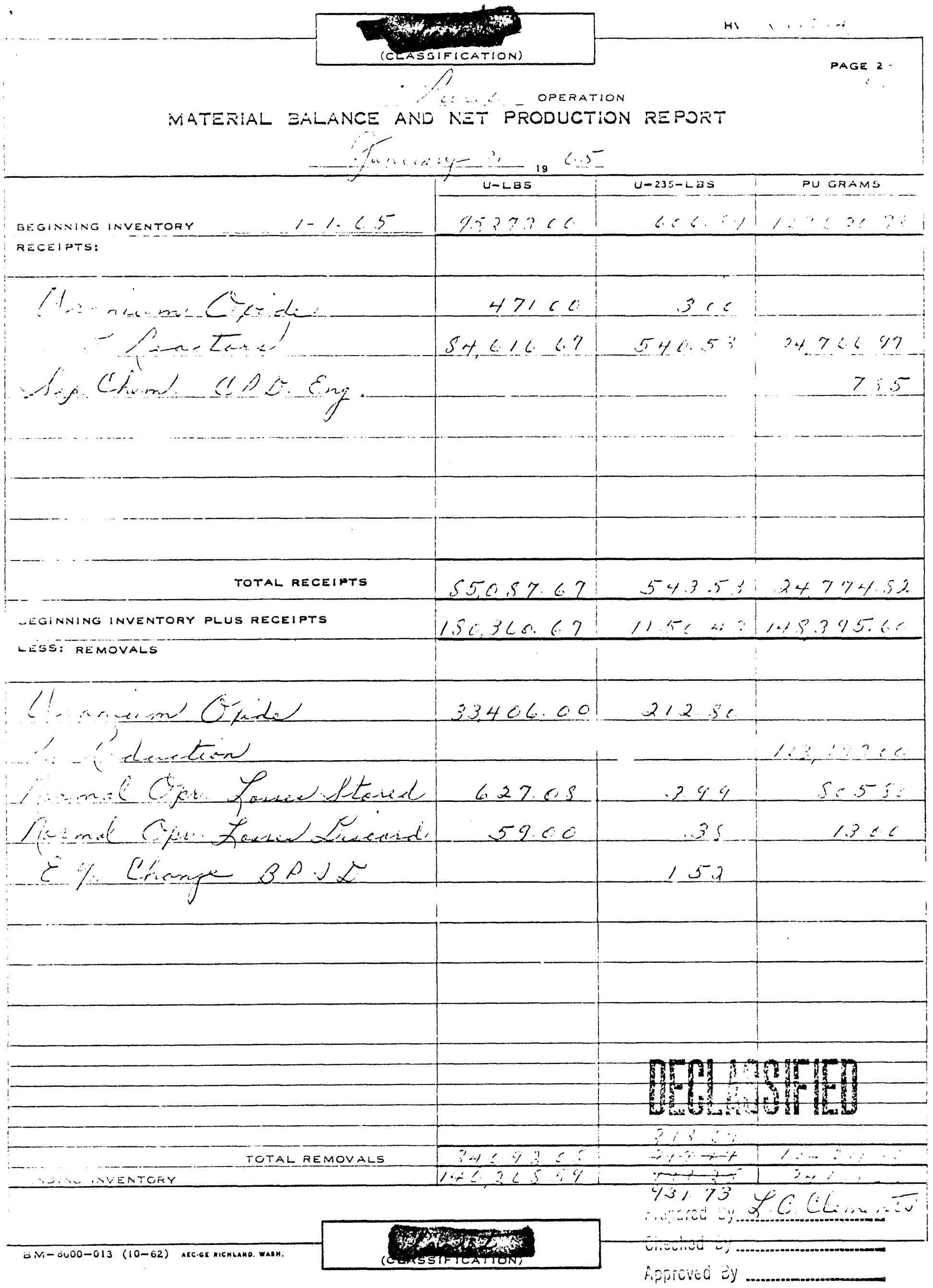




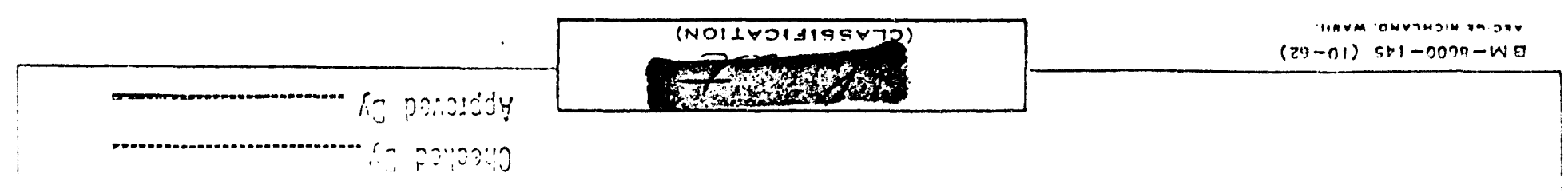

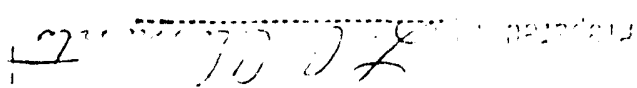

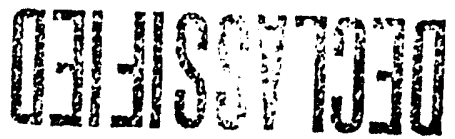

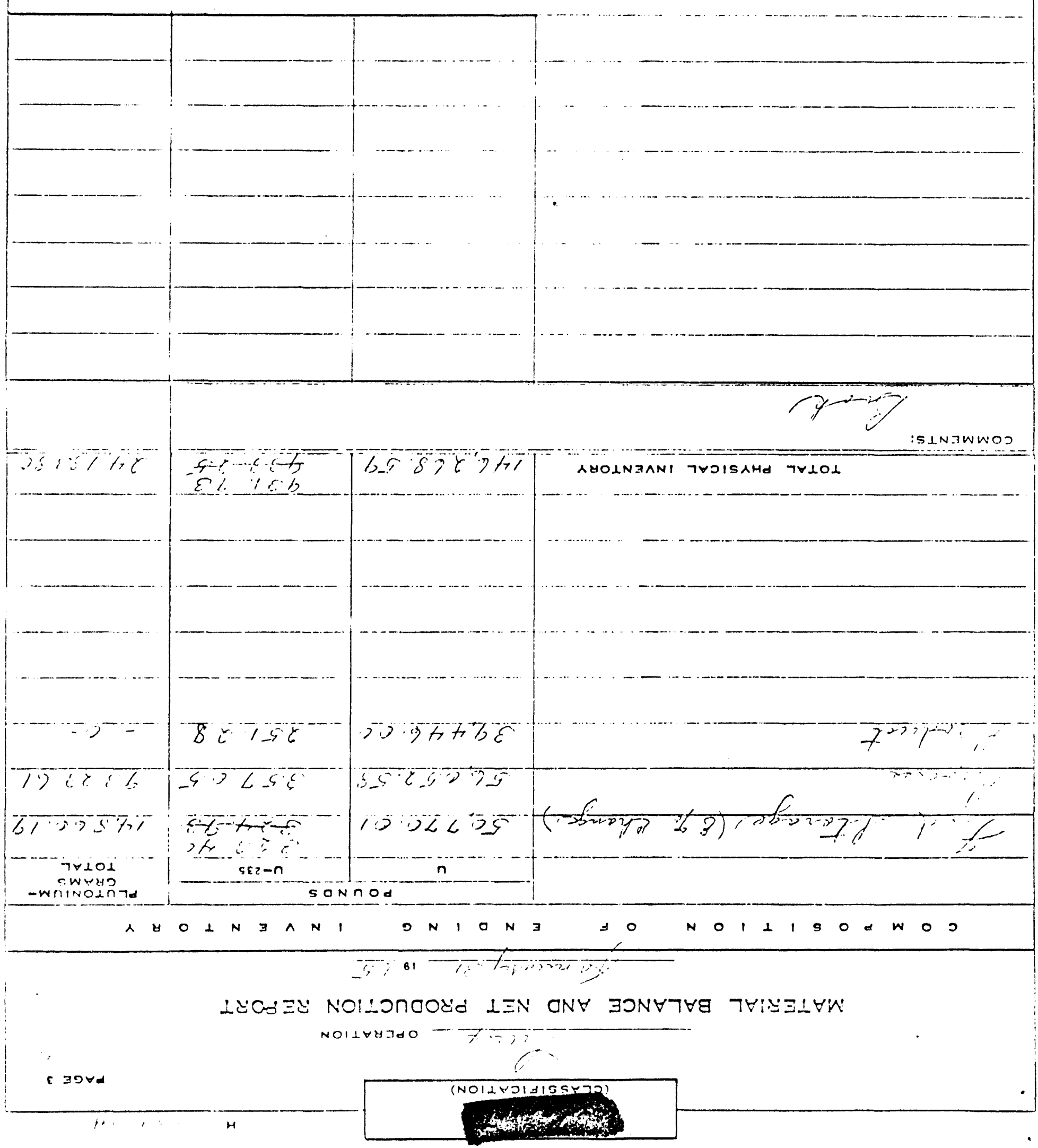




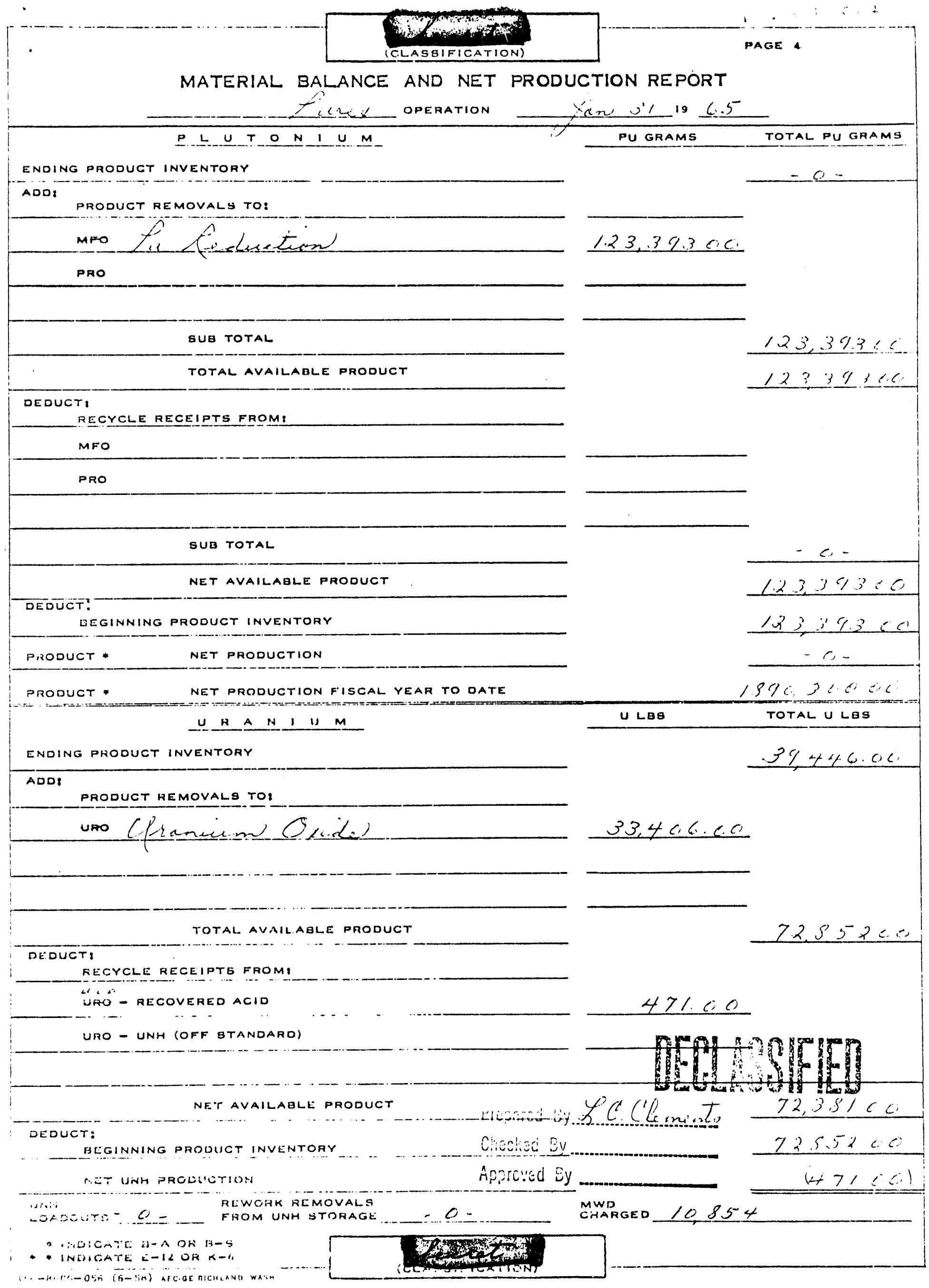




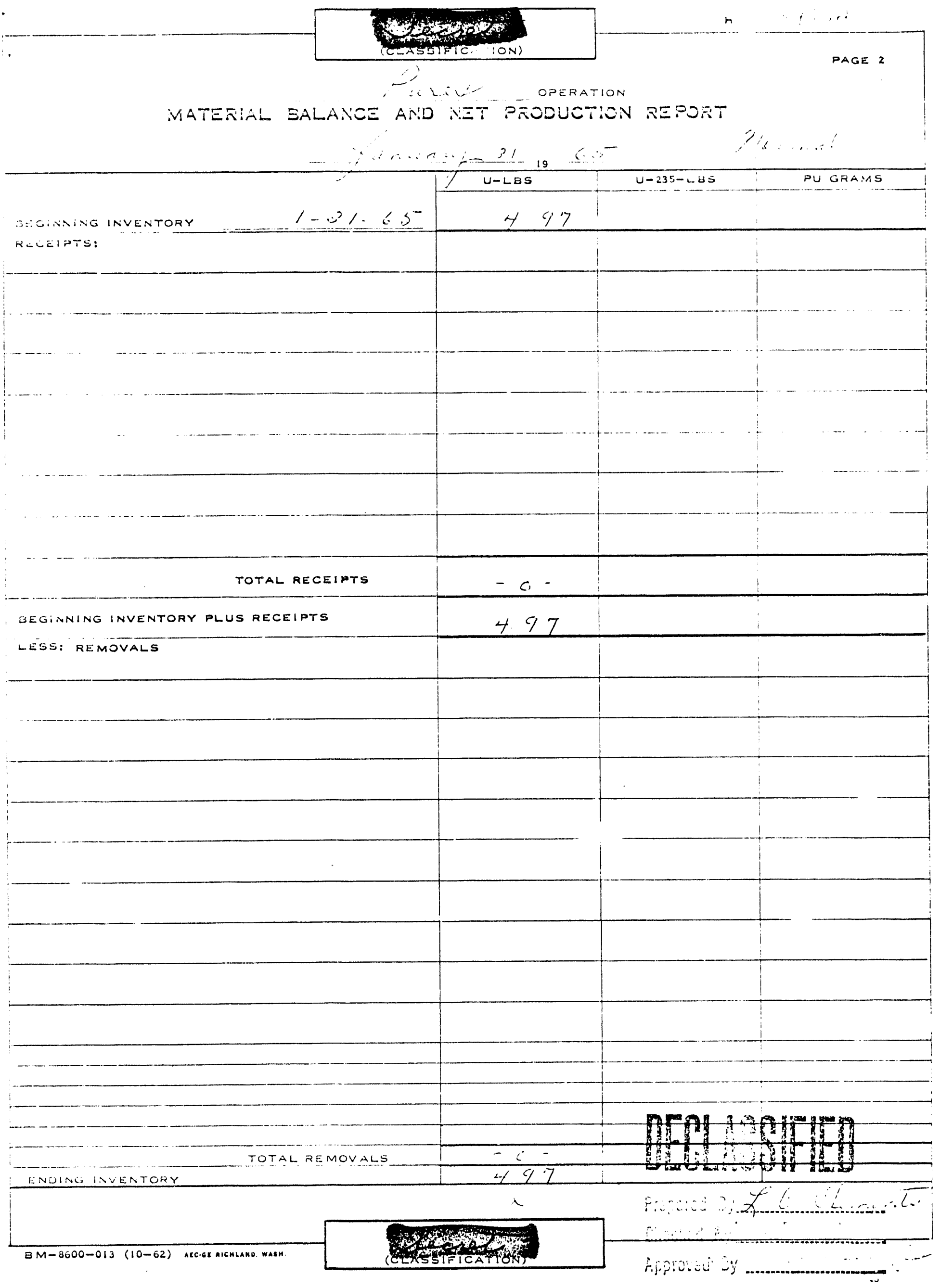




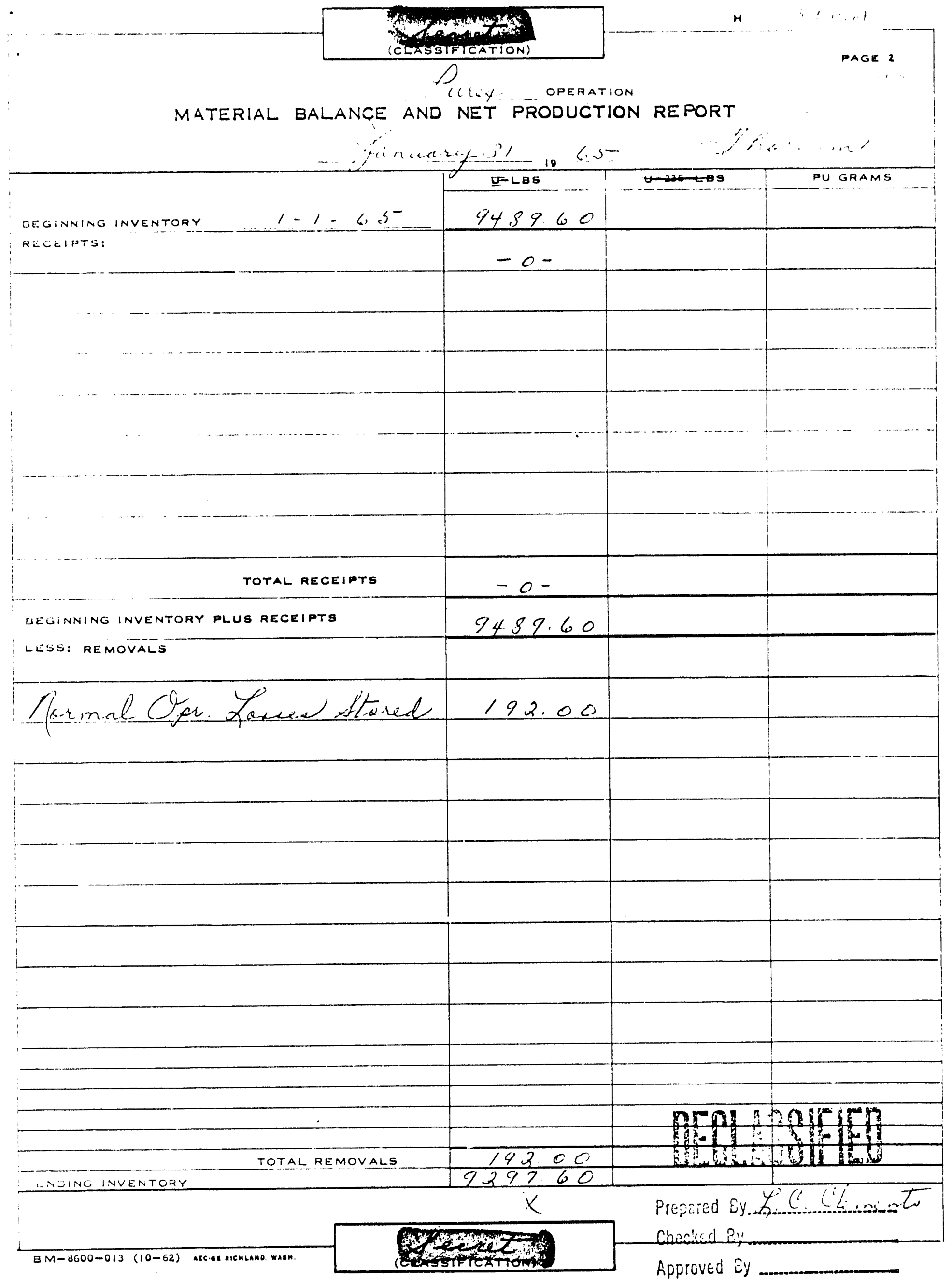




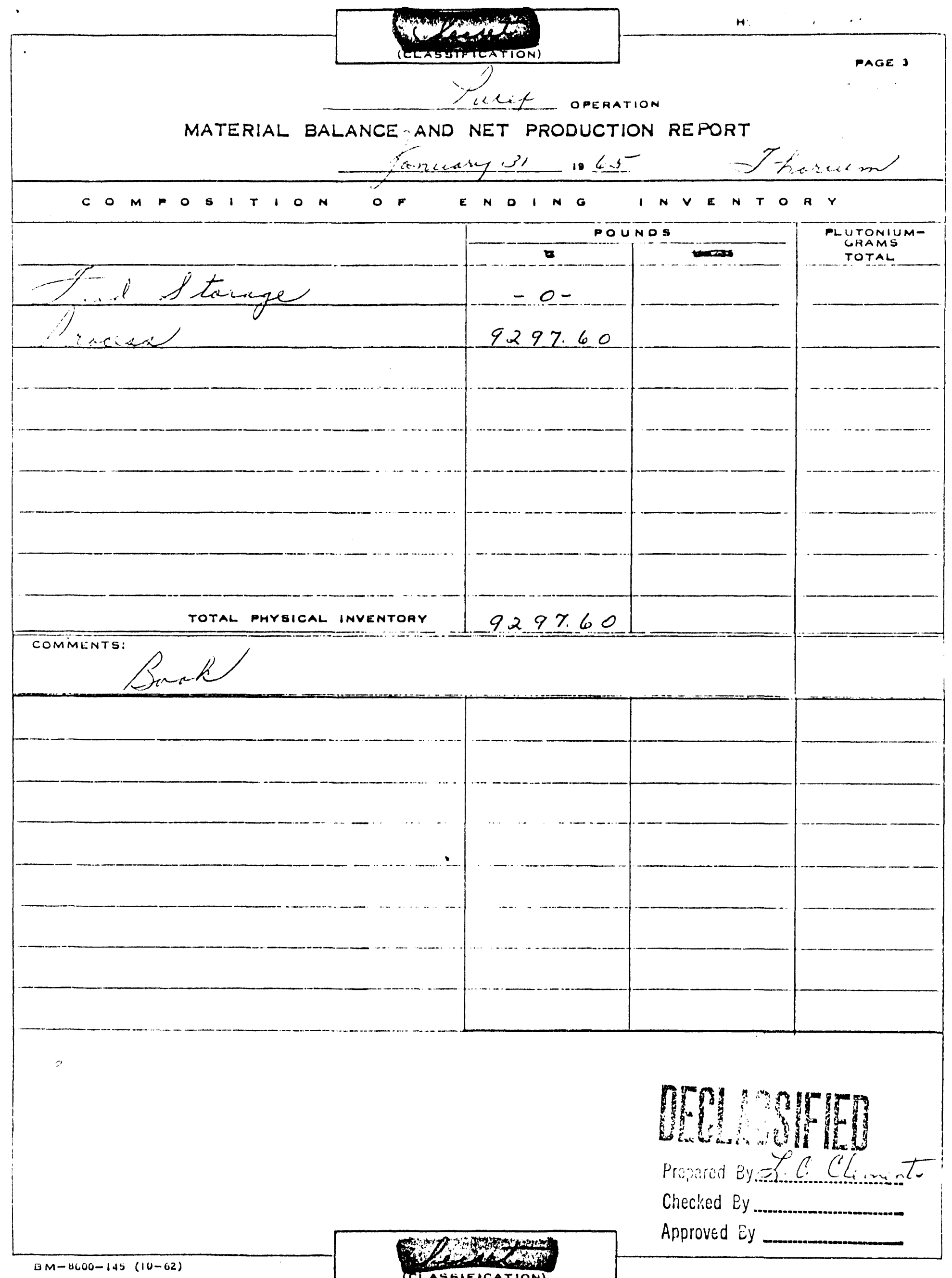




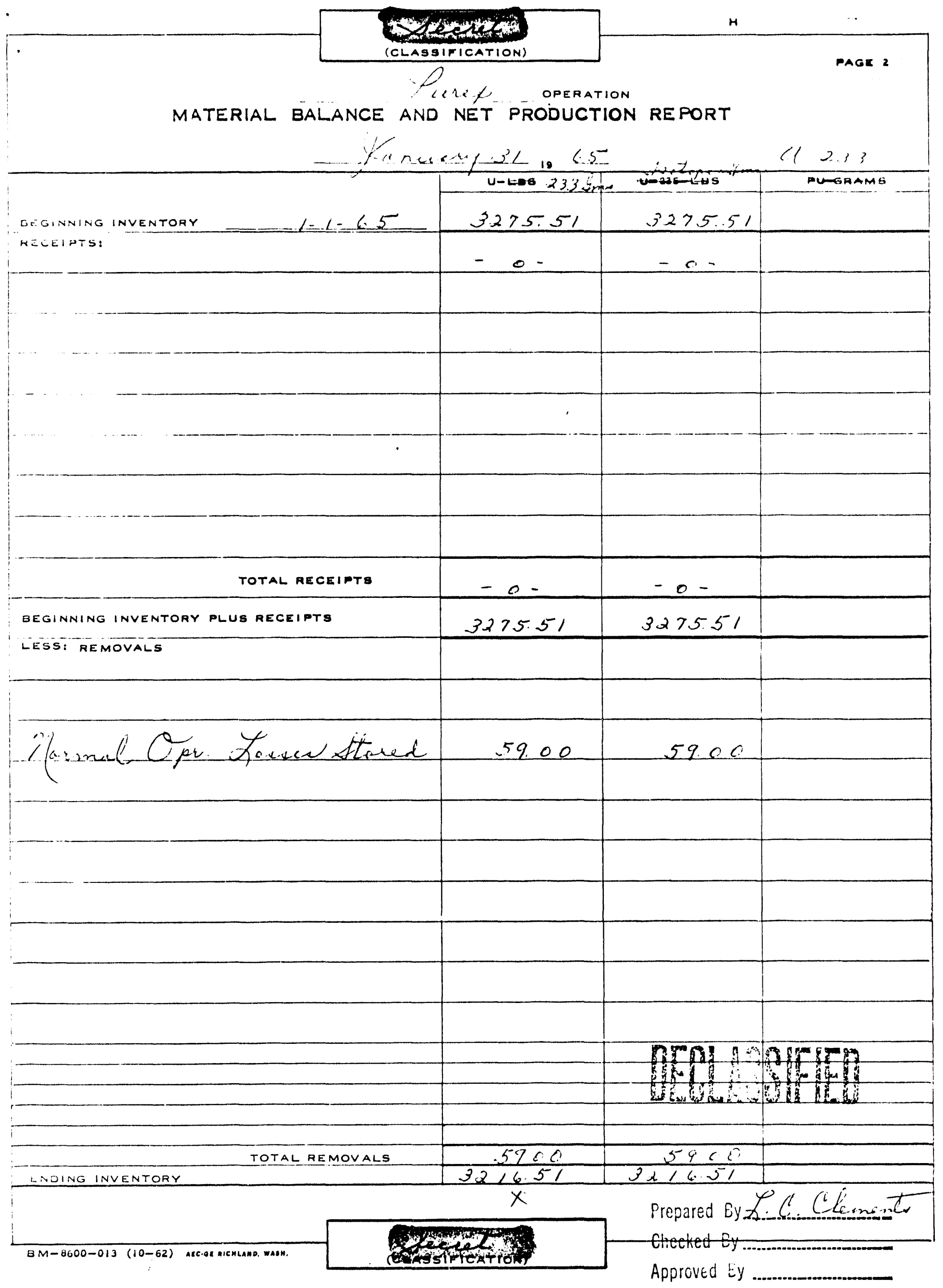




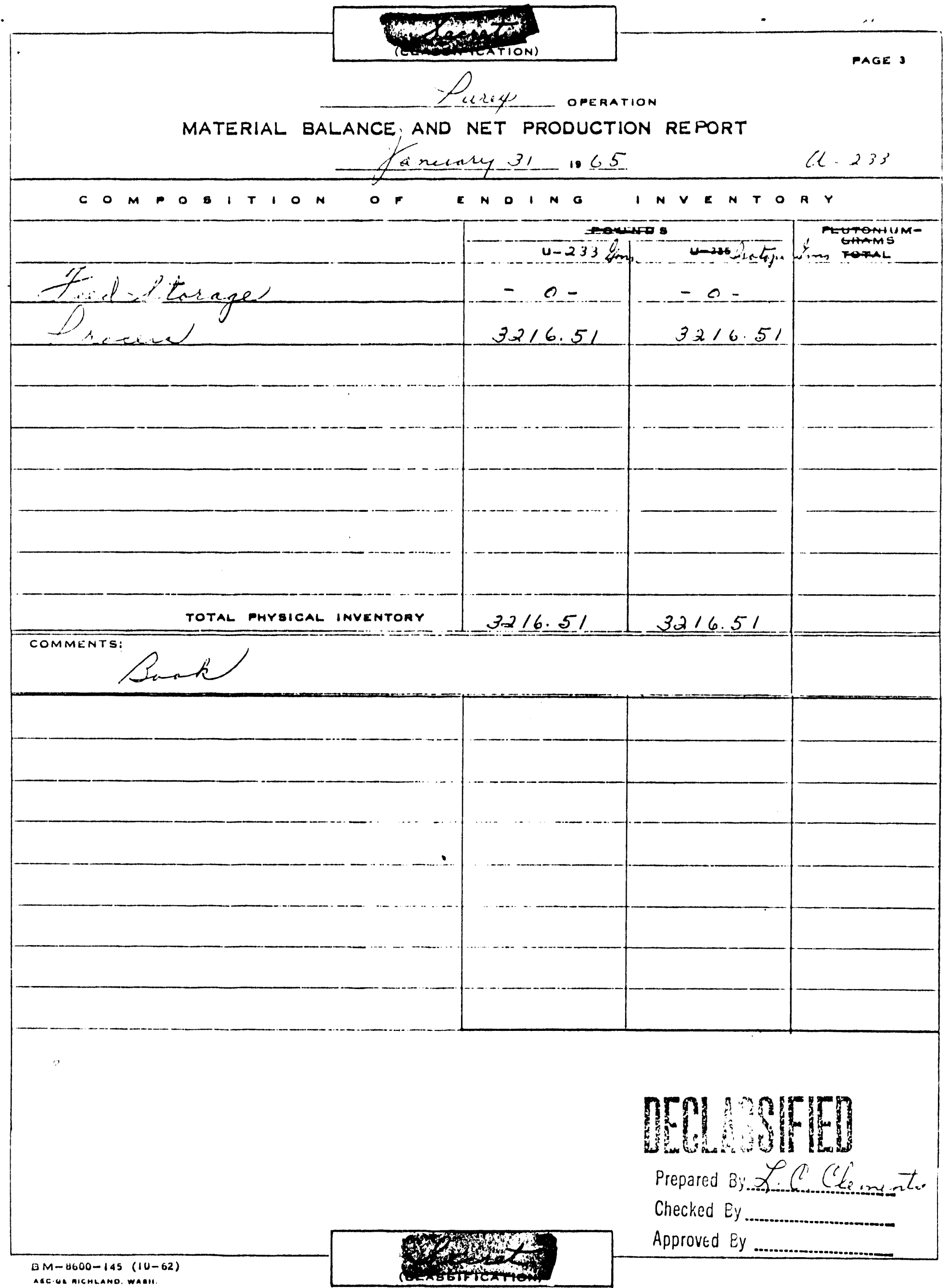


NW-

Pules operation

PAGE 2

MATERIAL BALANCE AND NET PRODUCTION REPORT

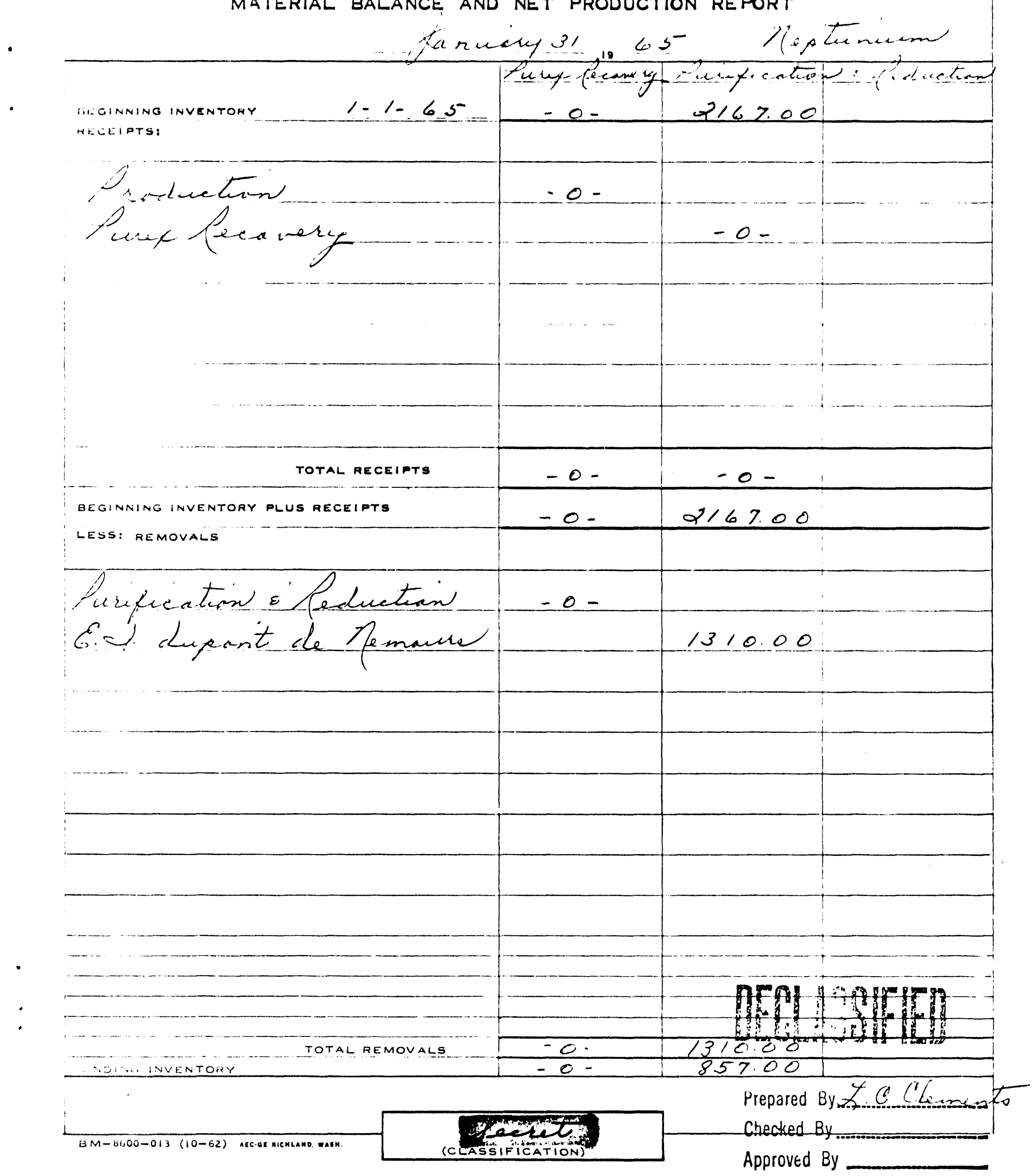




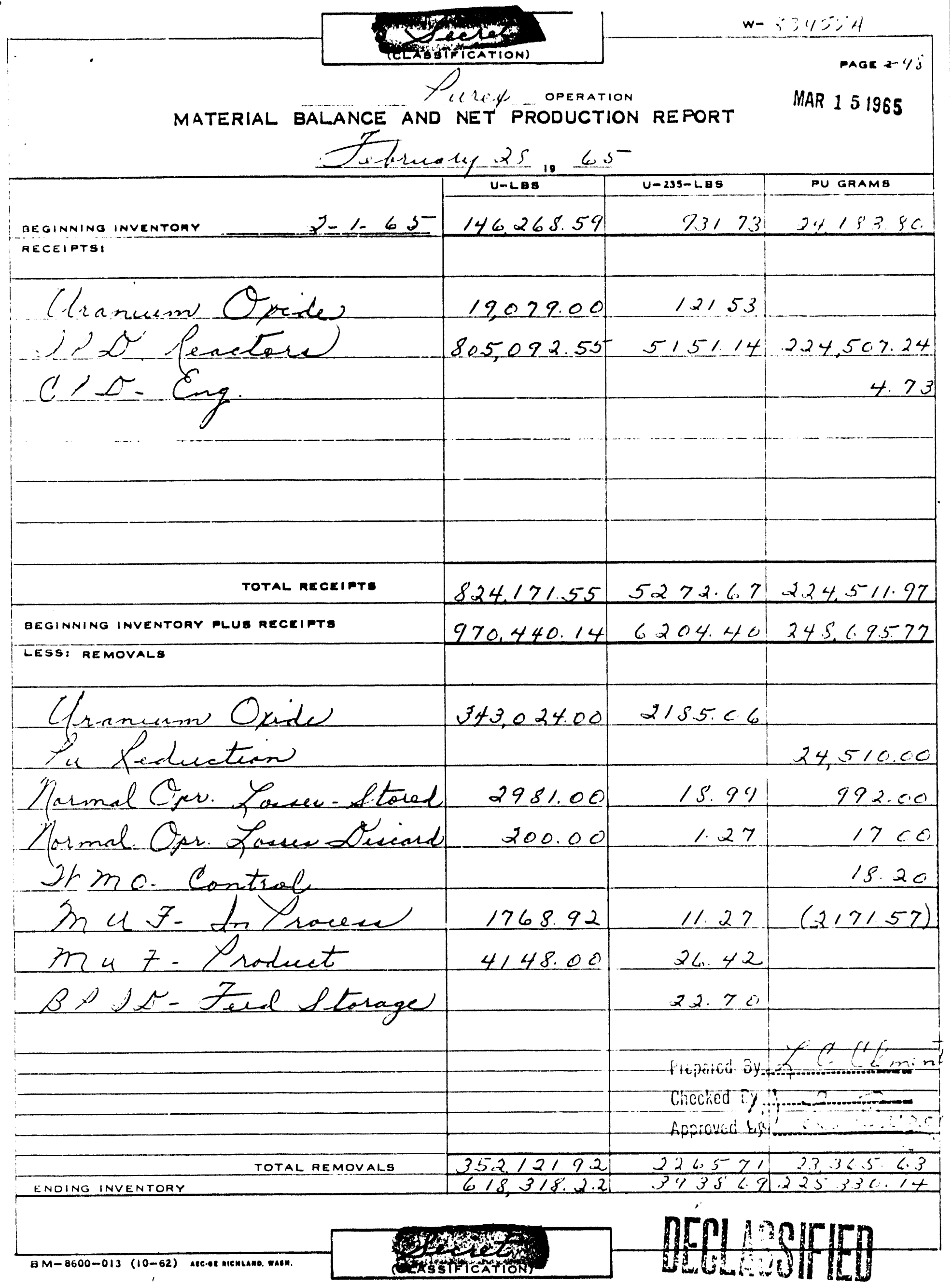




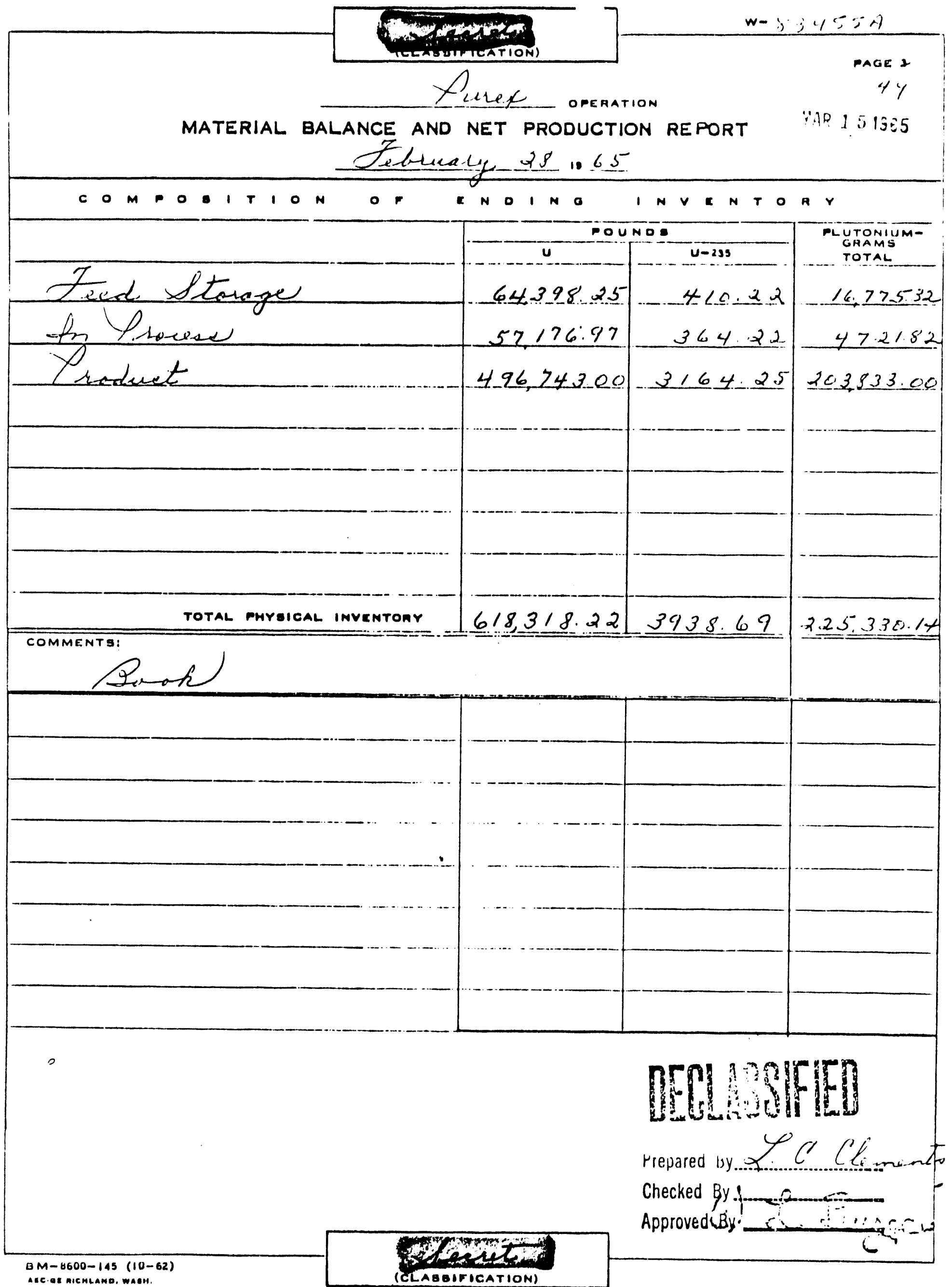




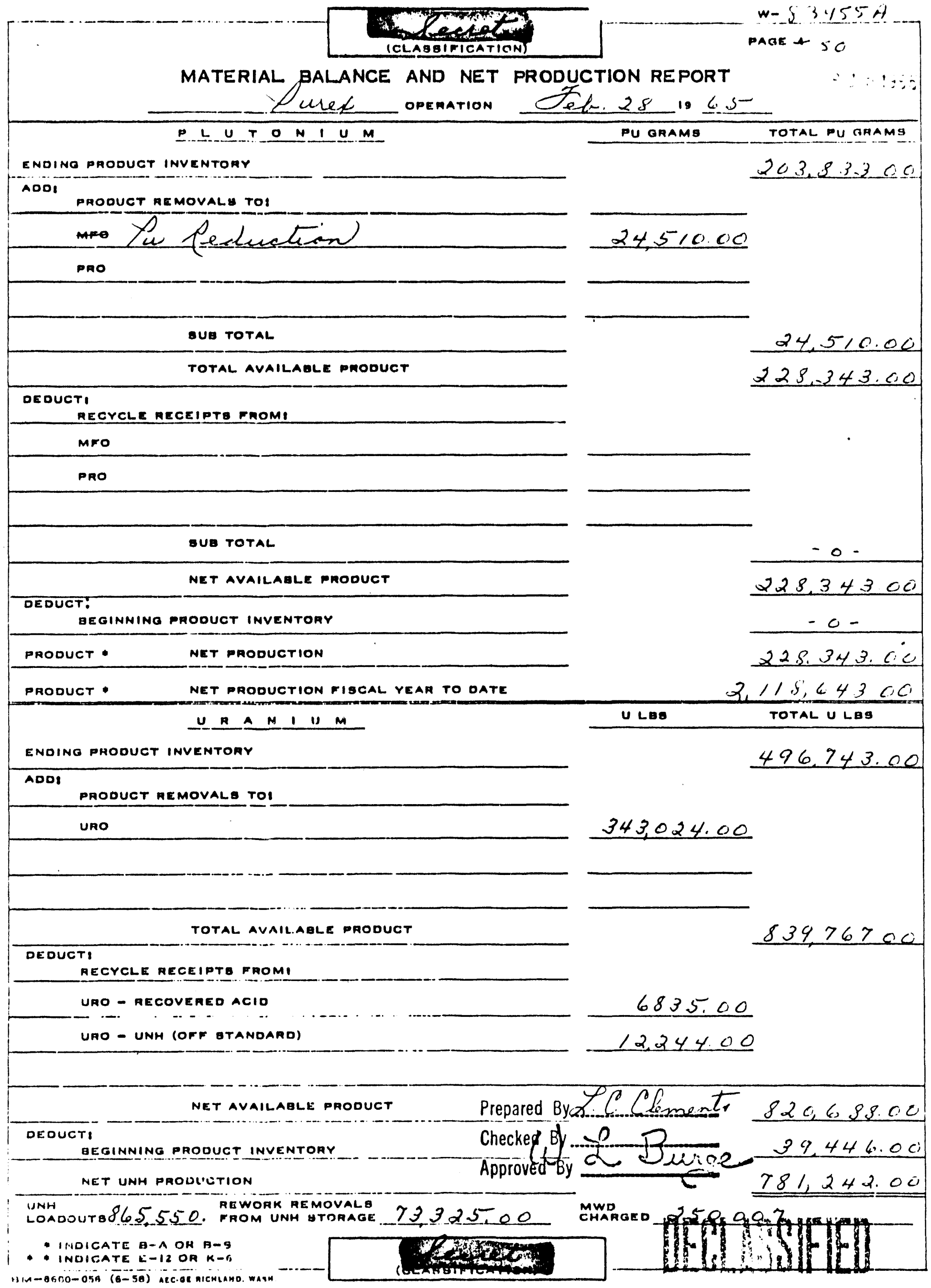




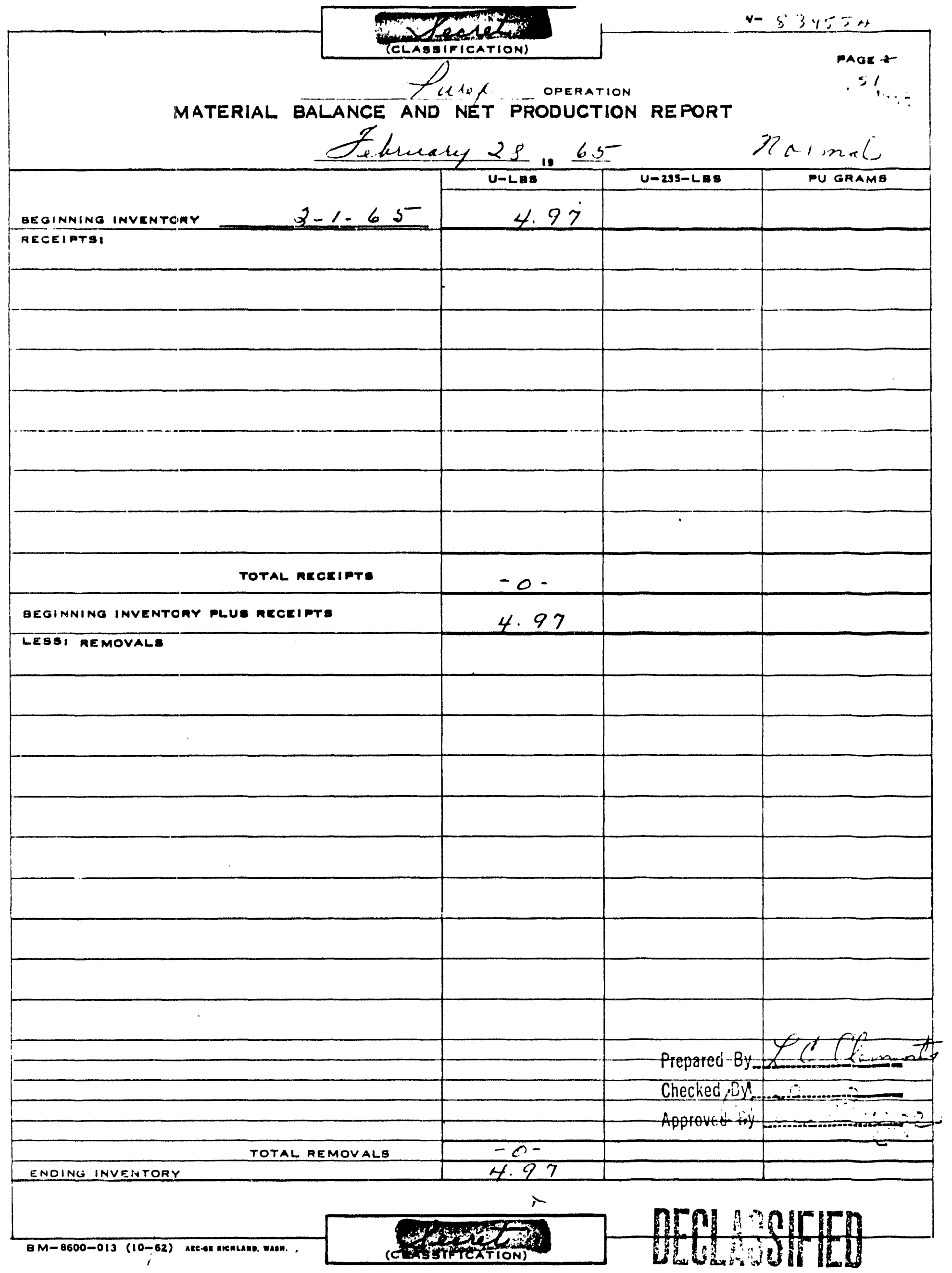




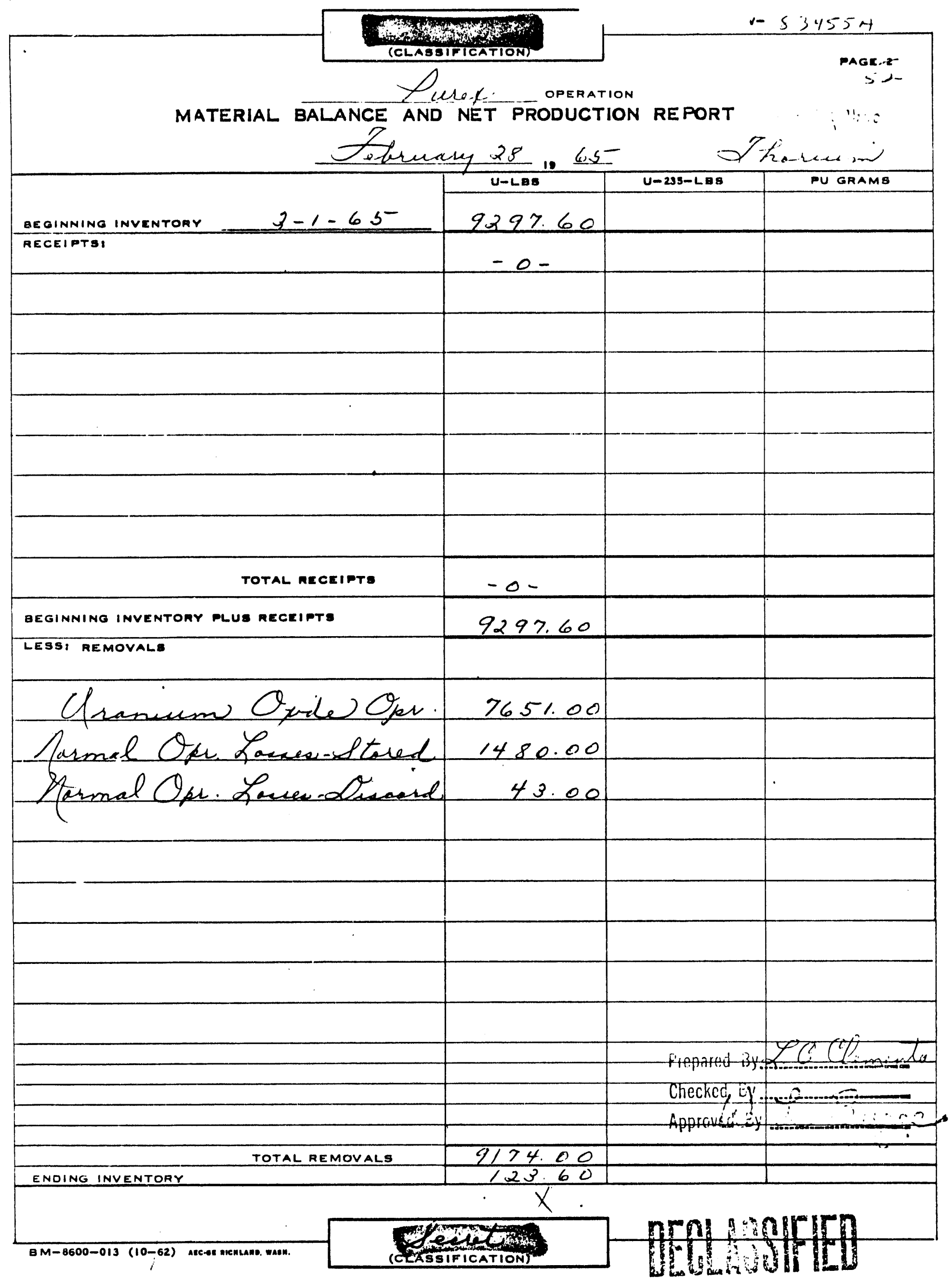




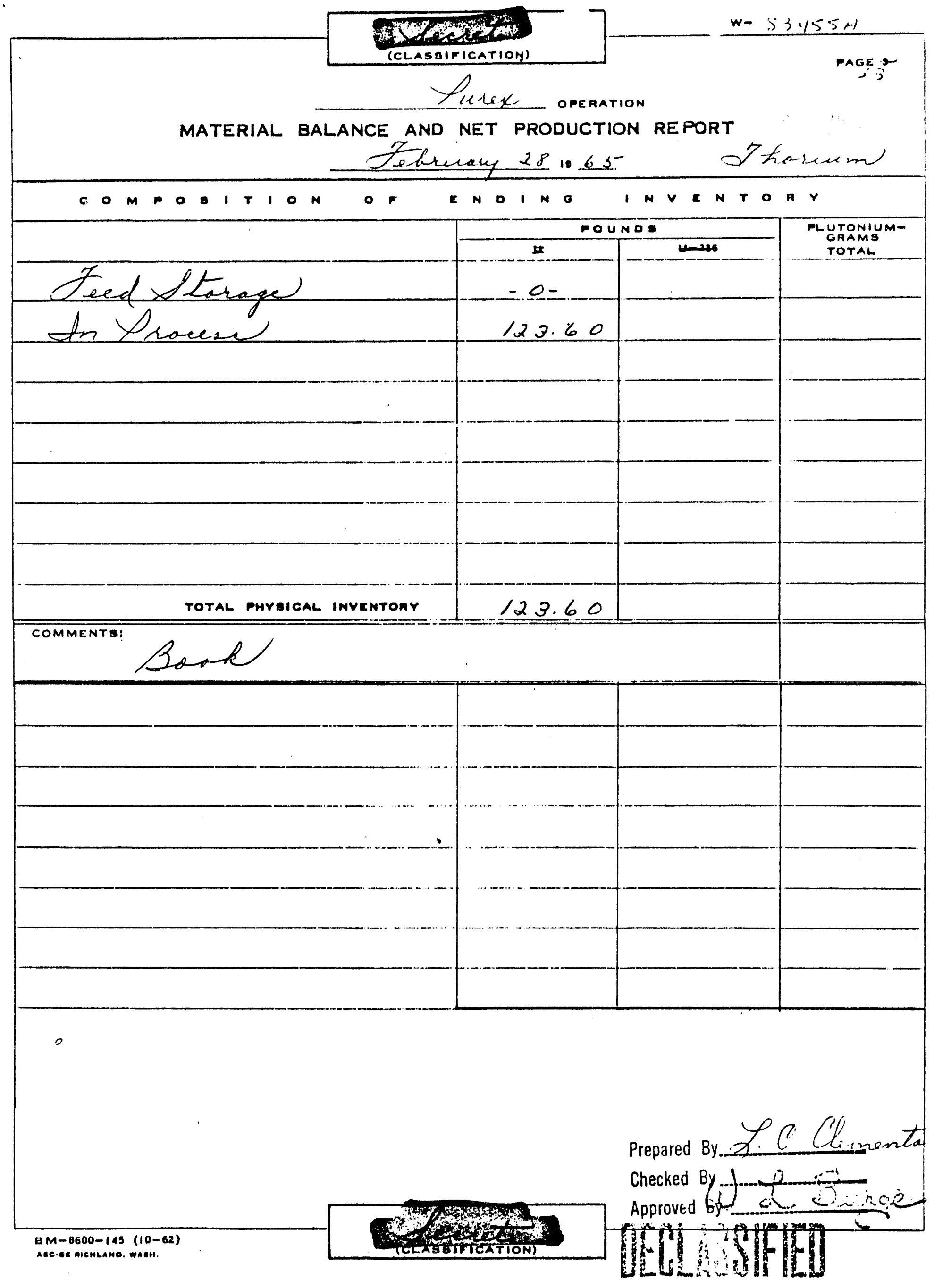




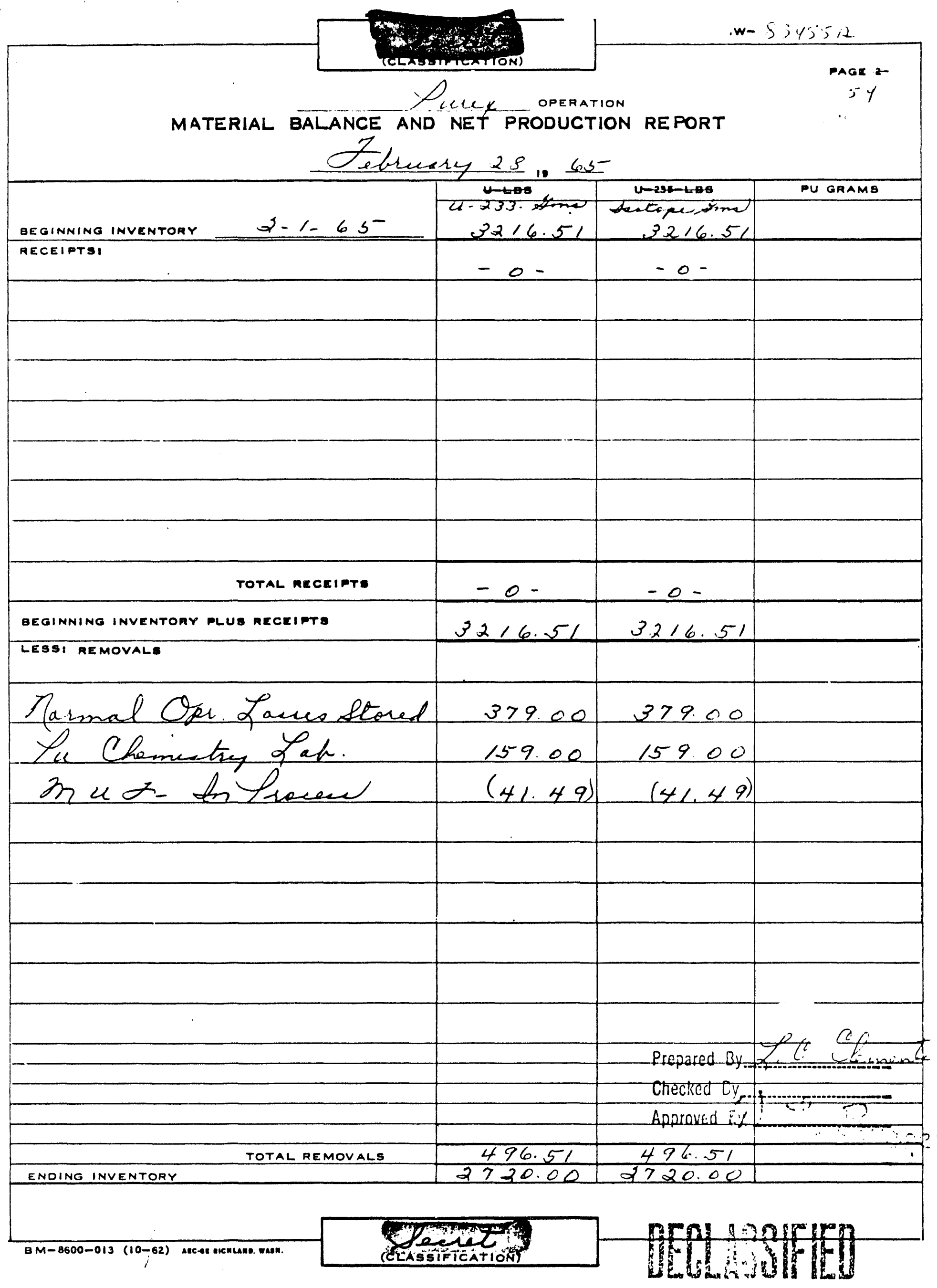




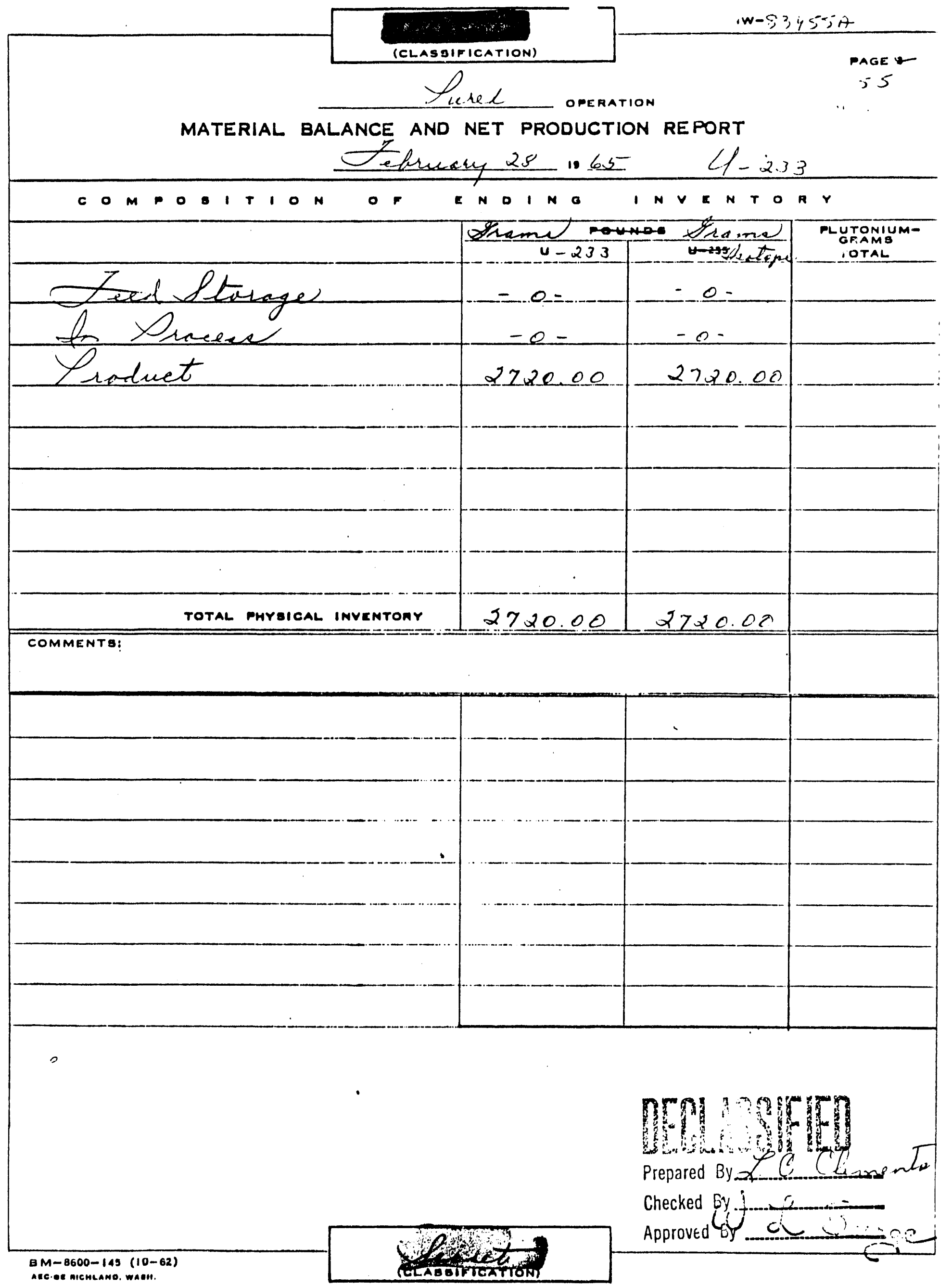




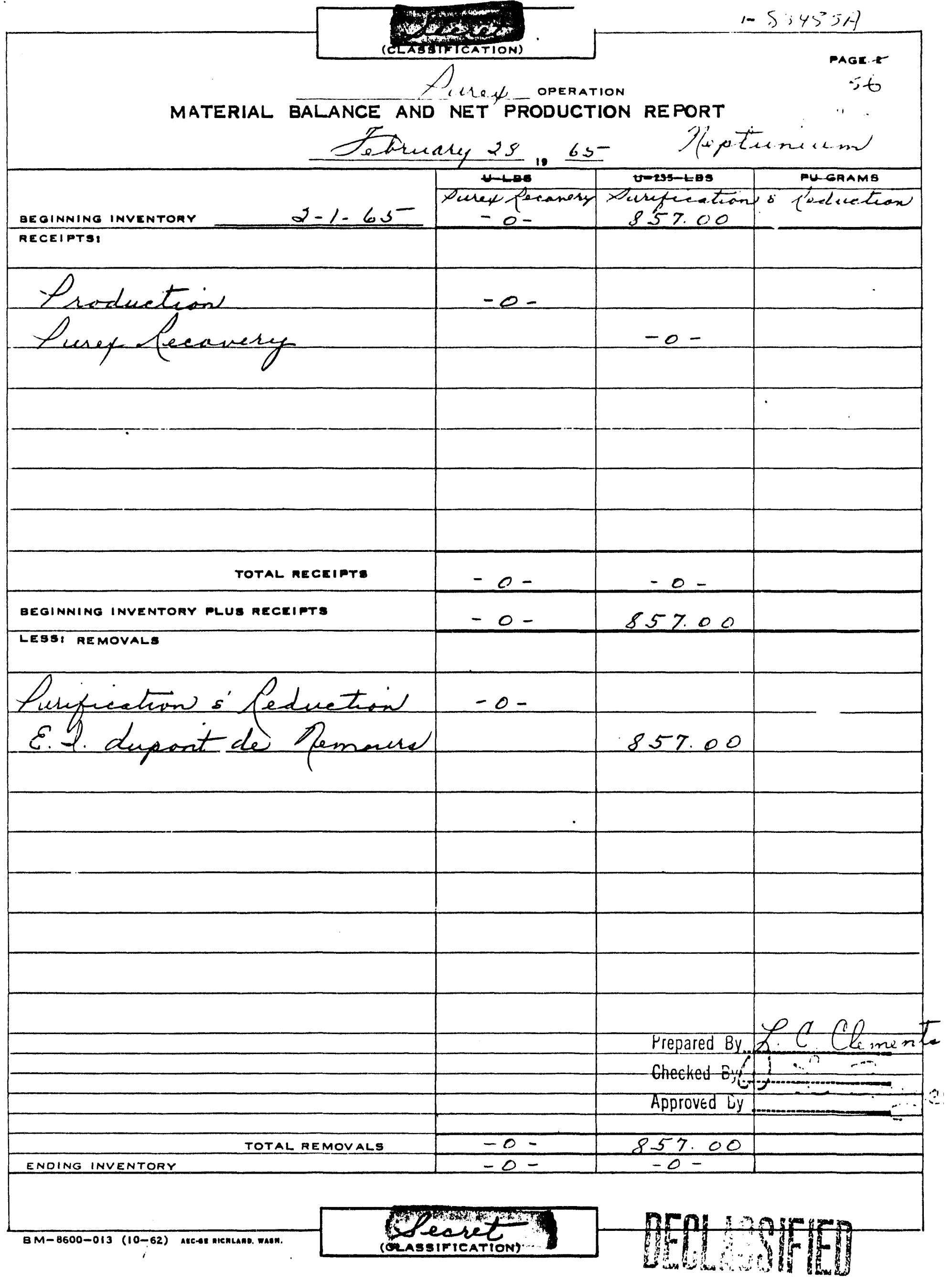




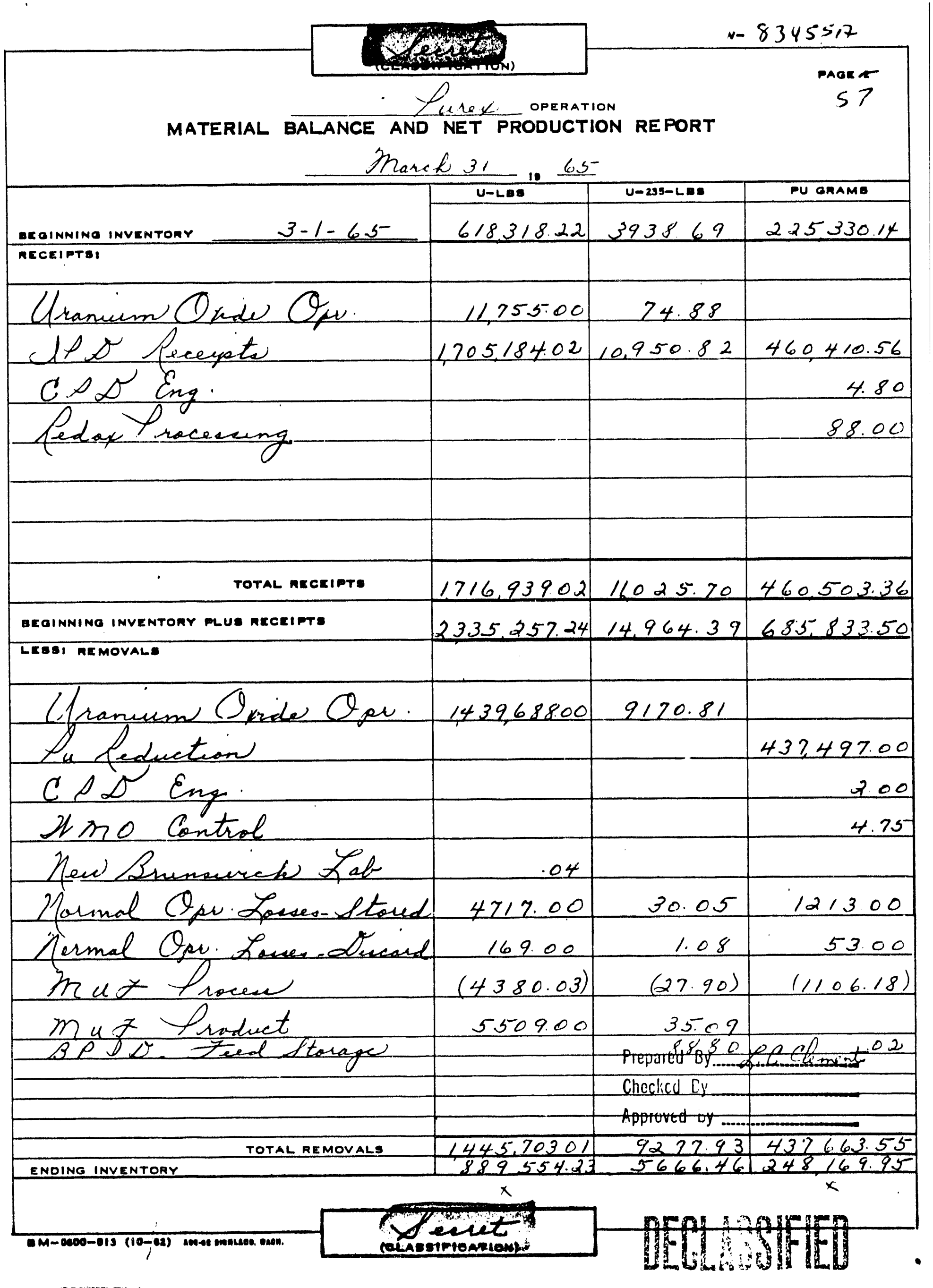




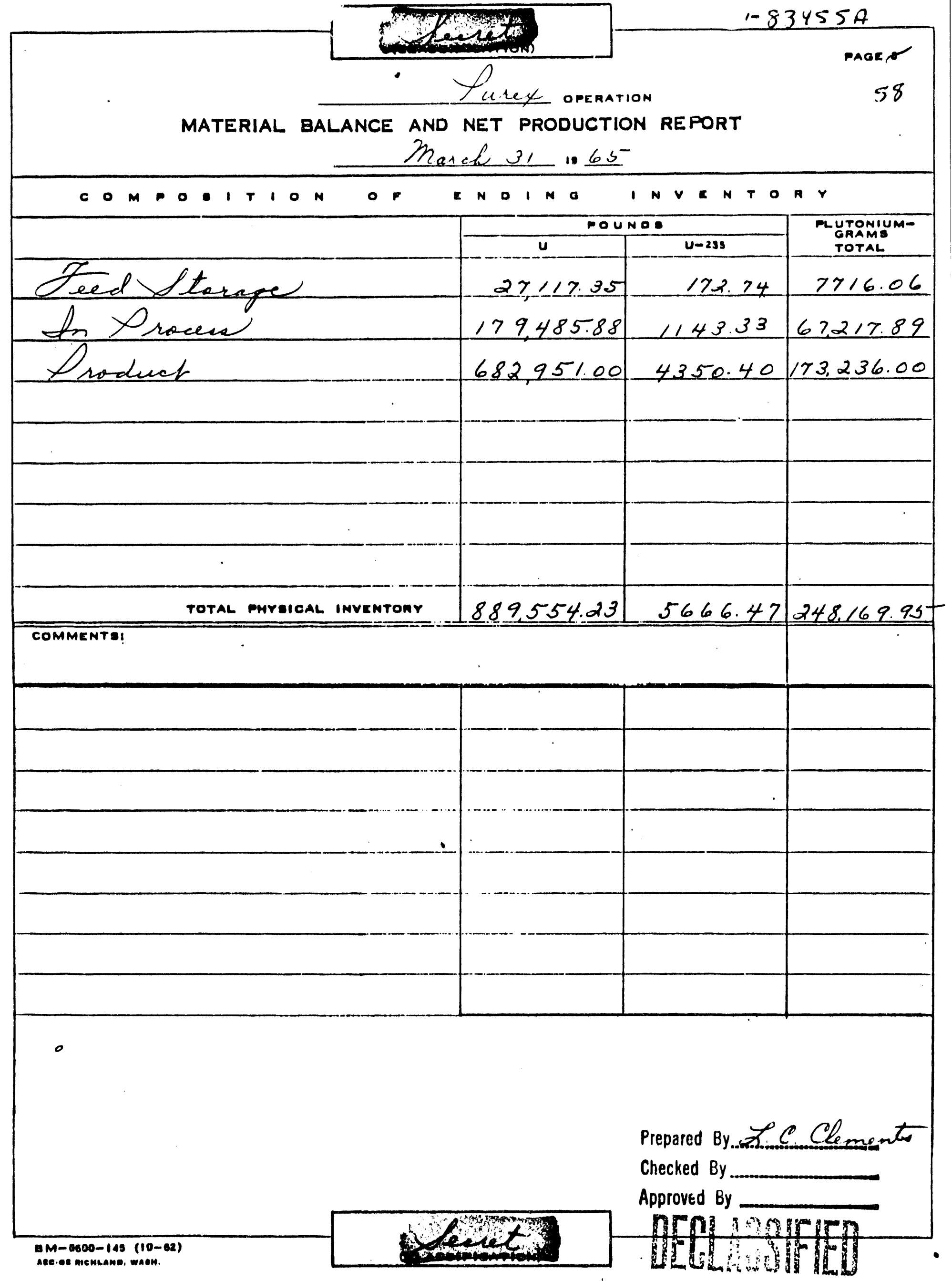




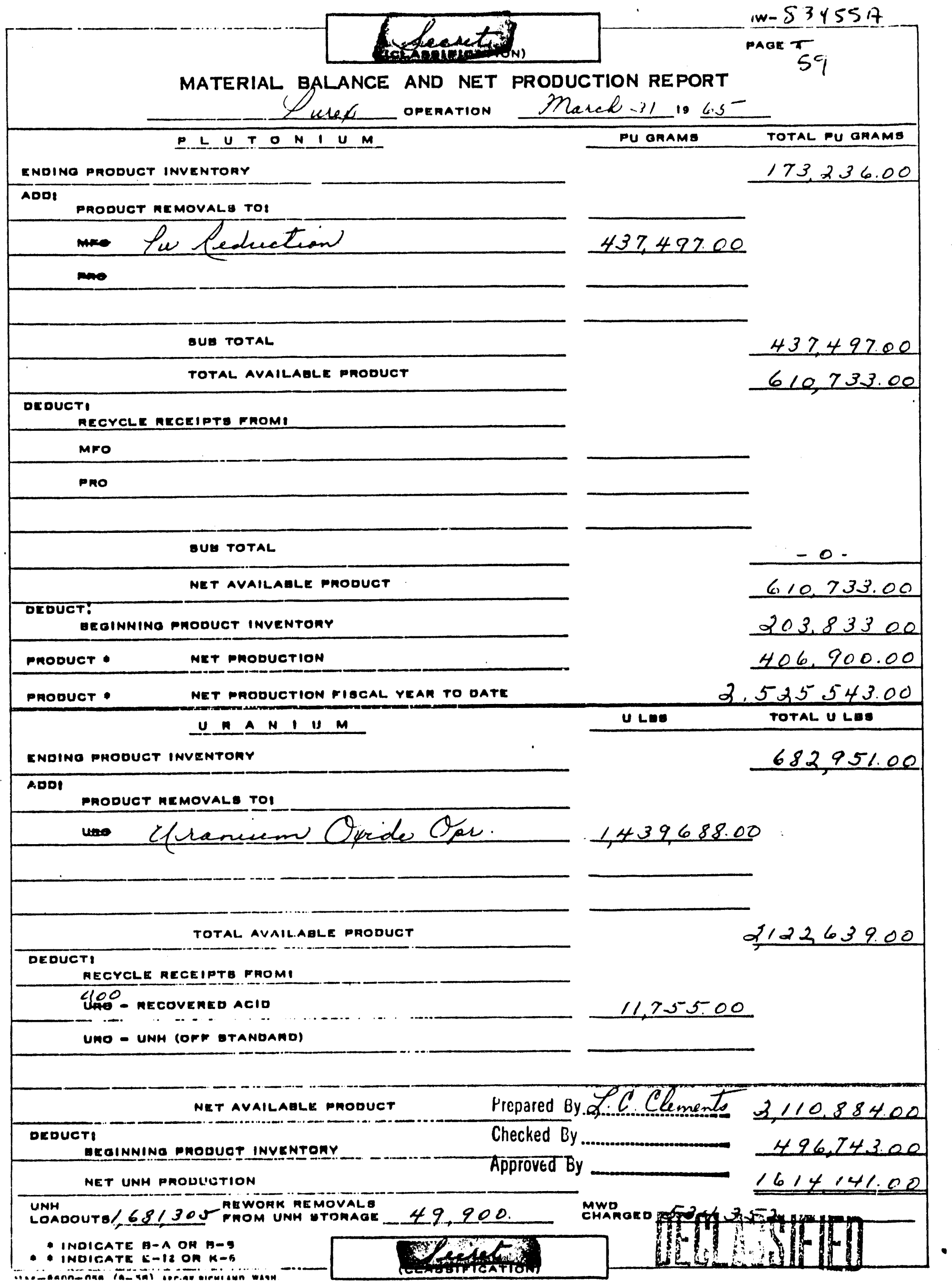




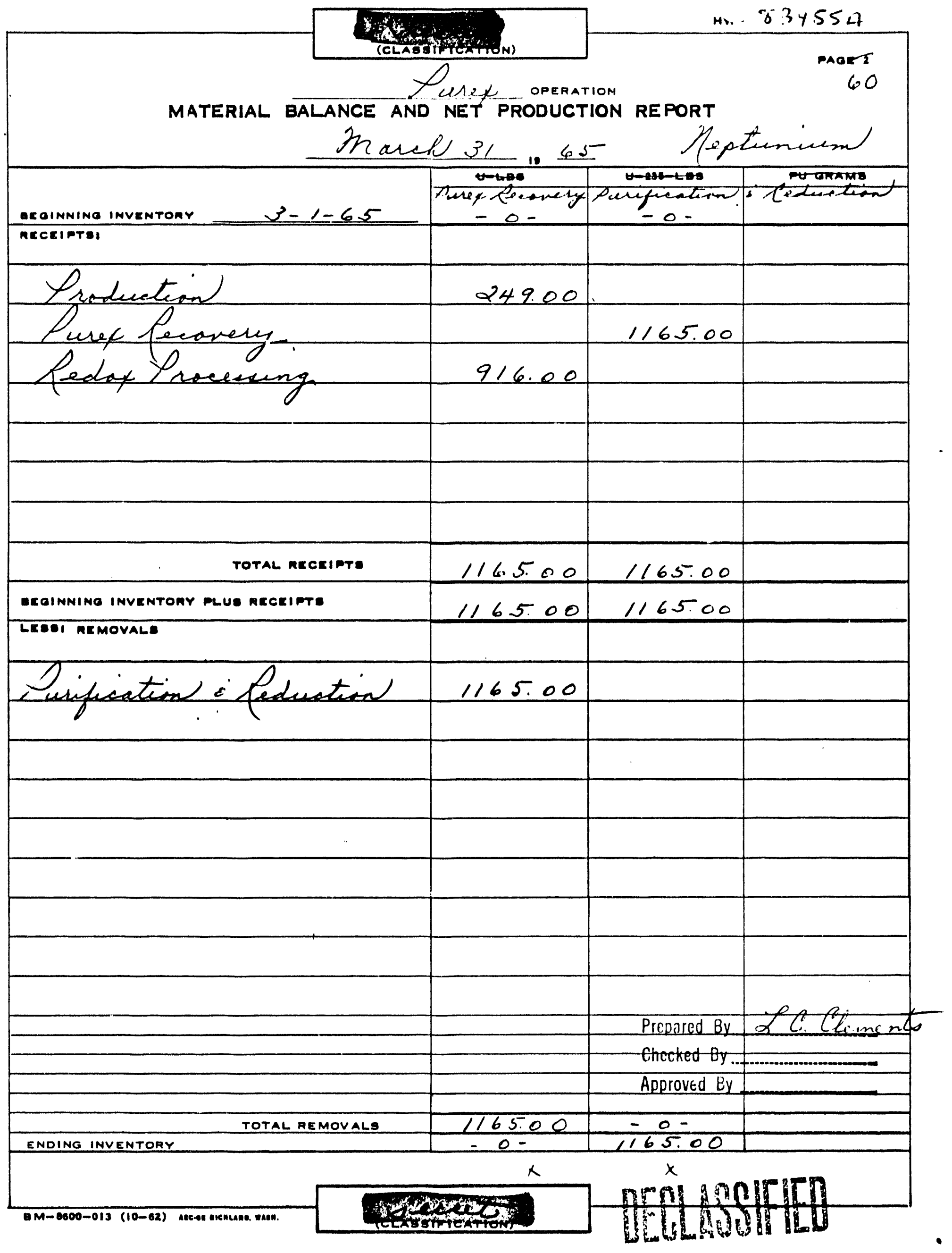




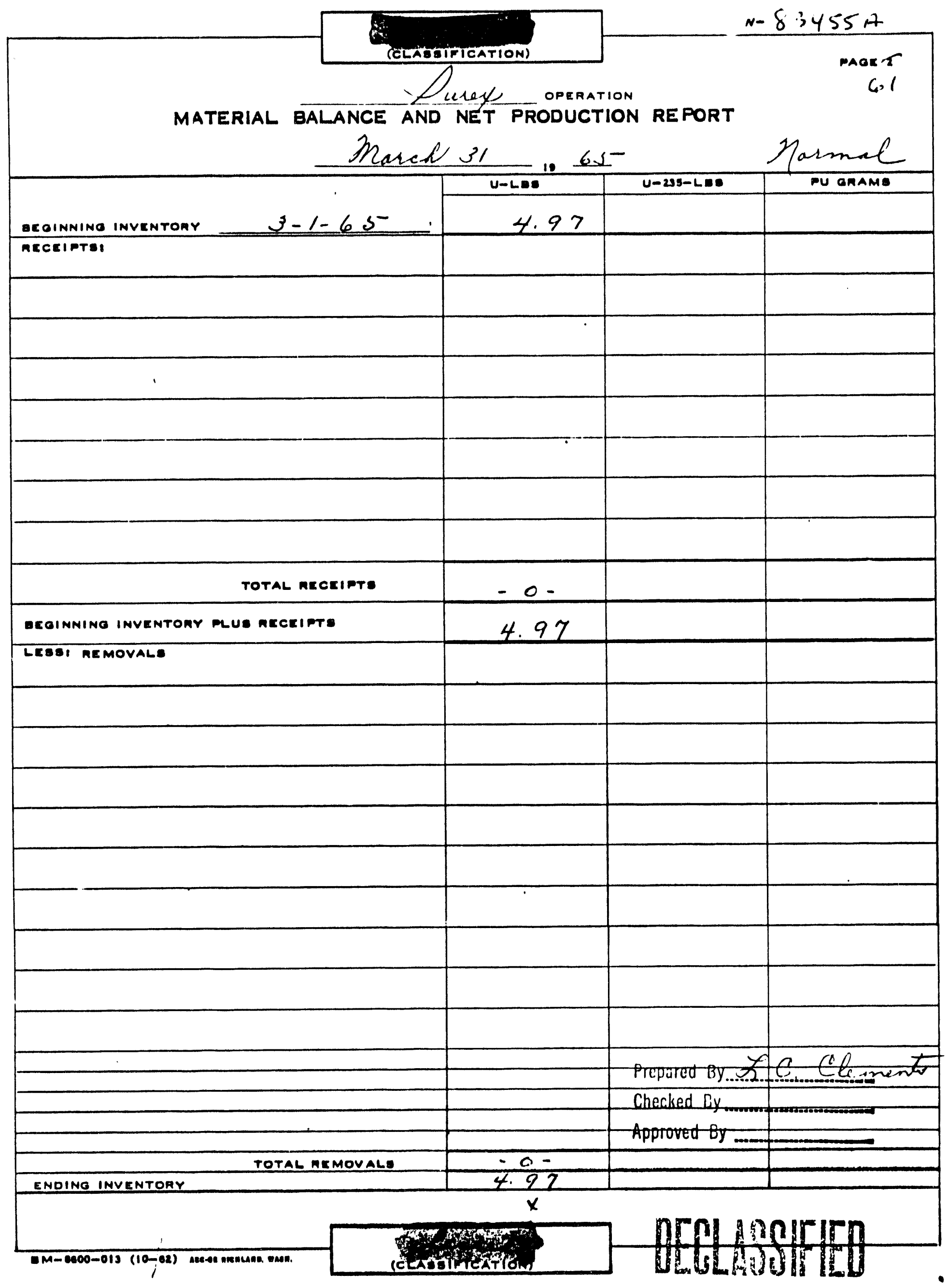




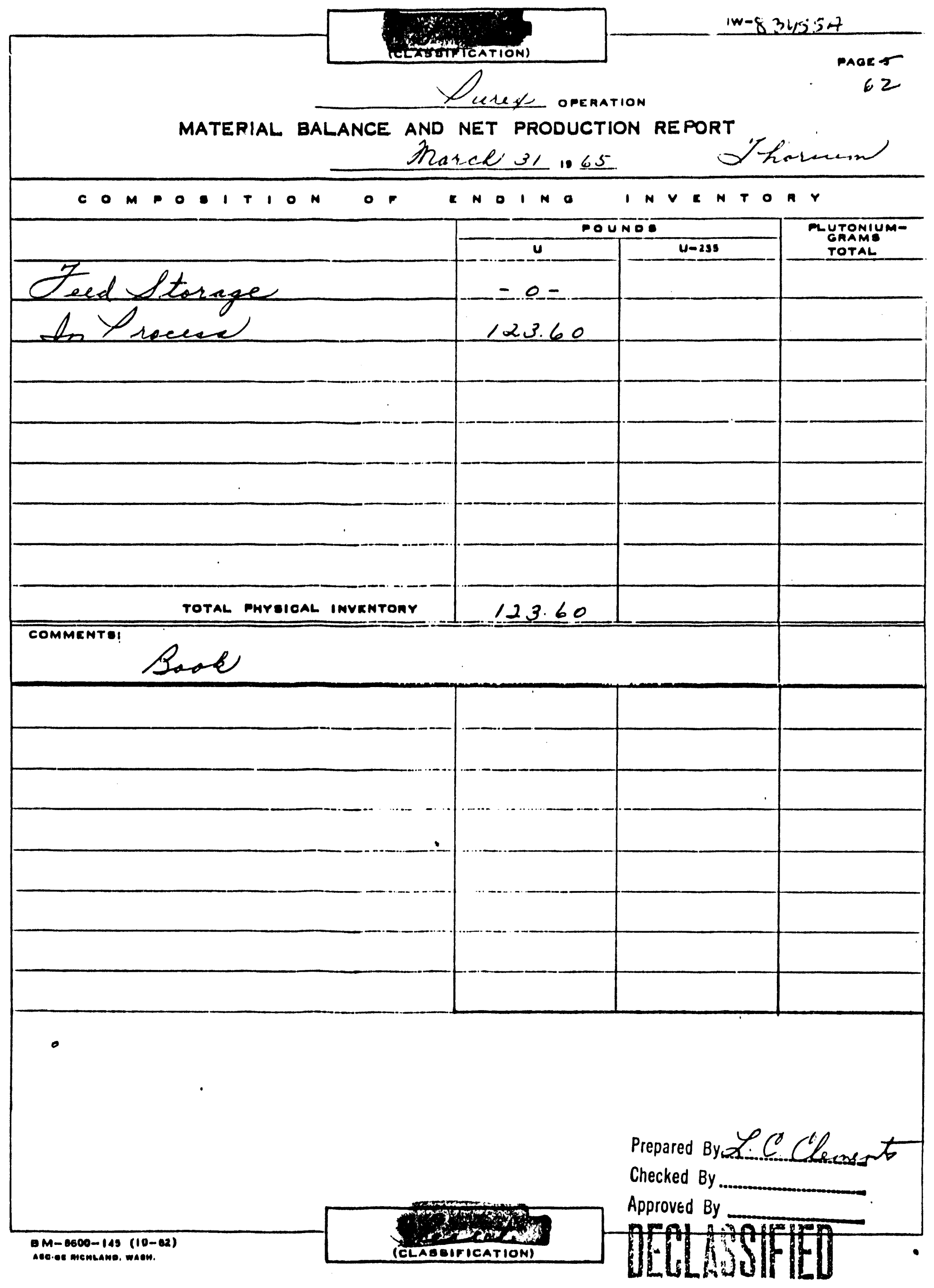




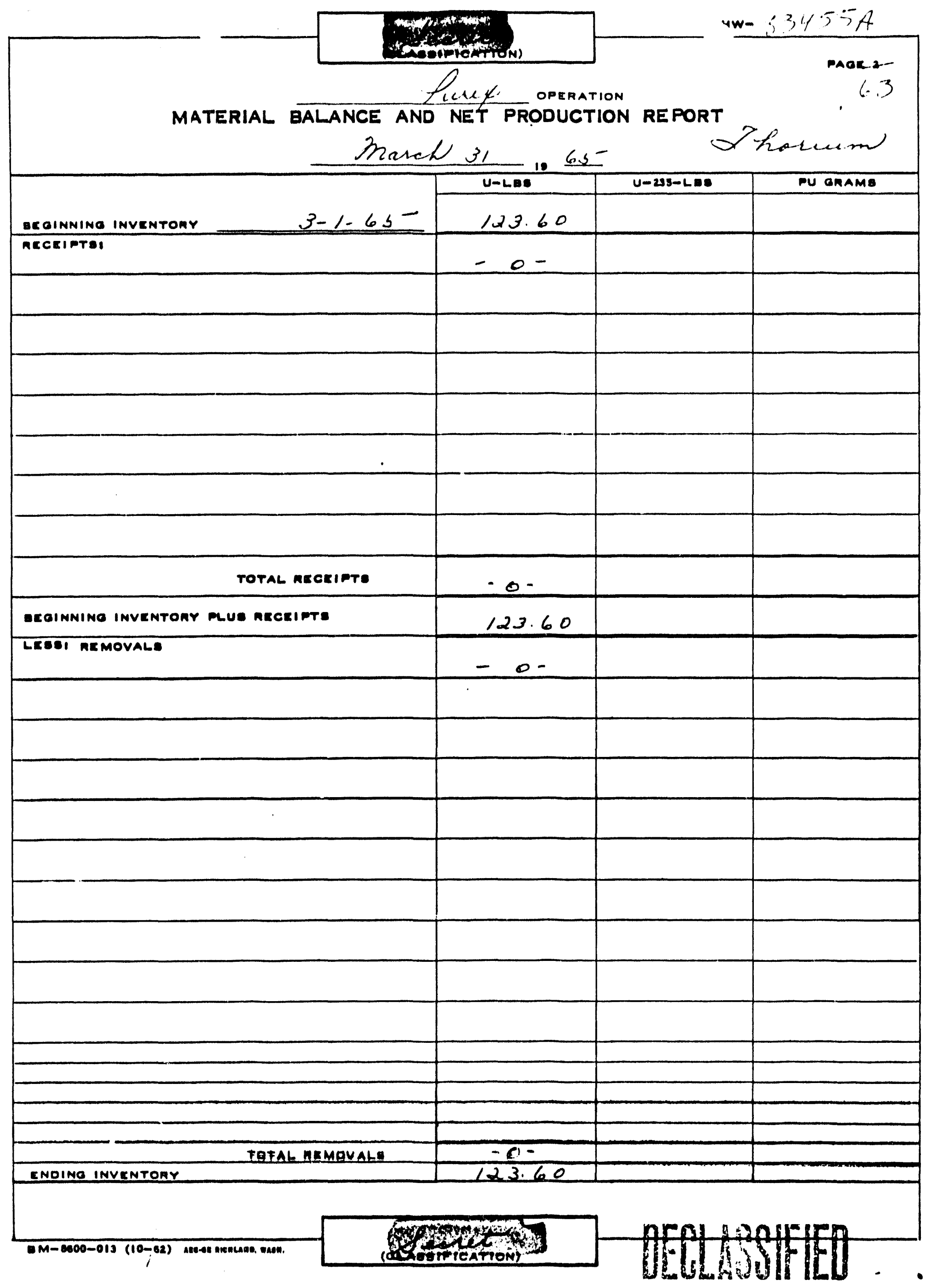




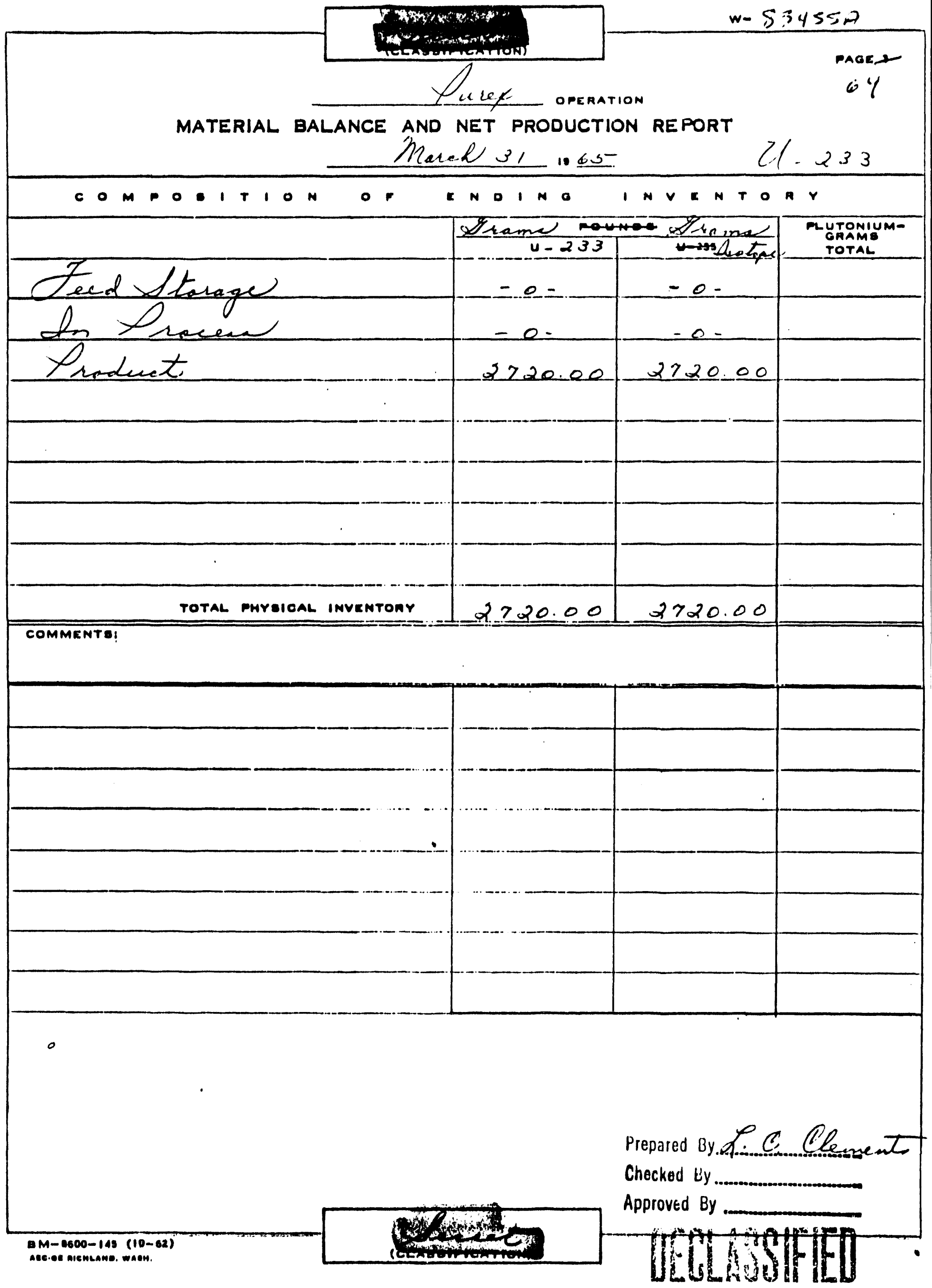




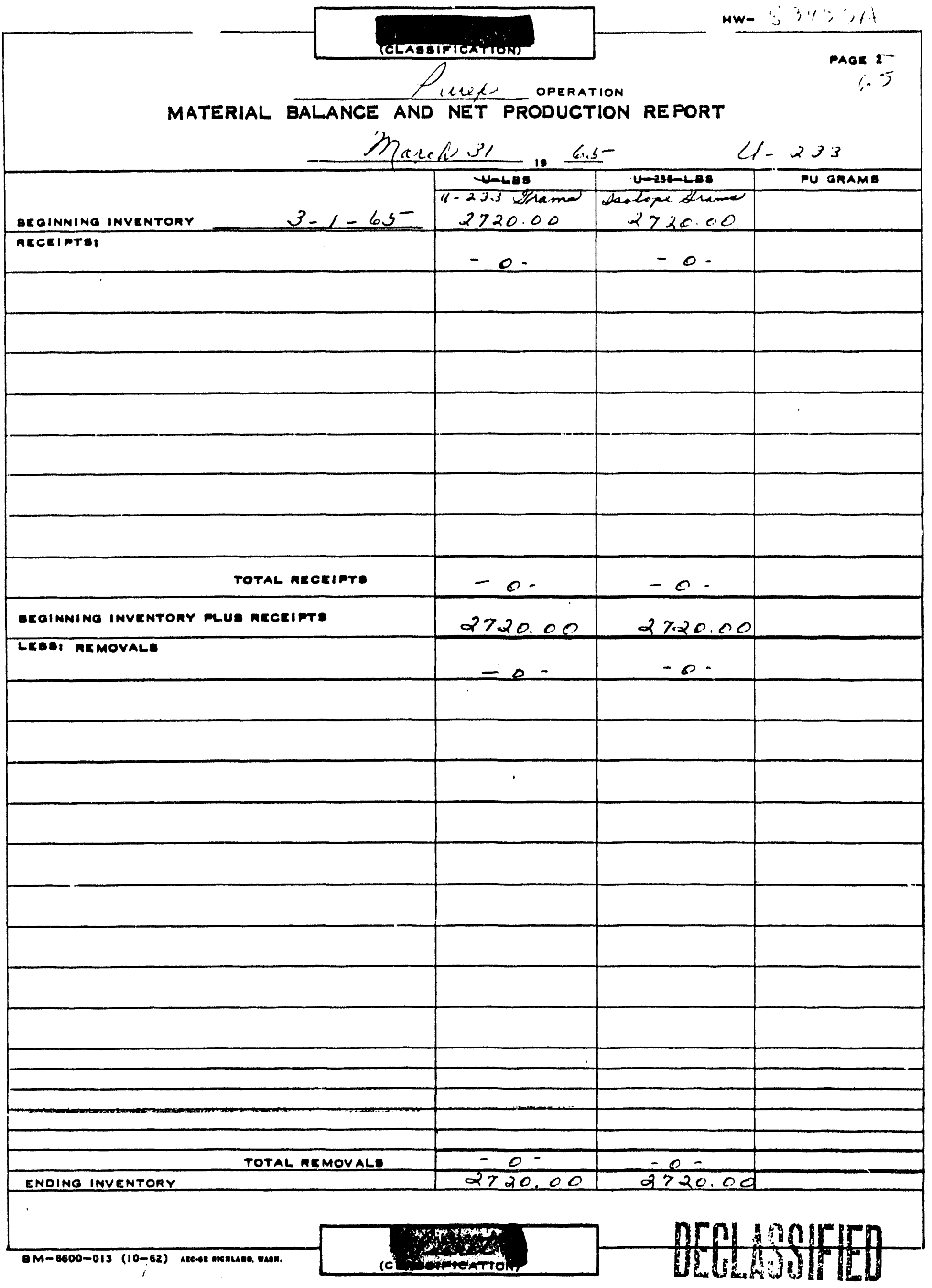




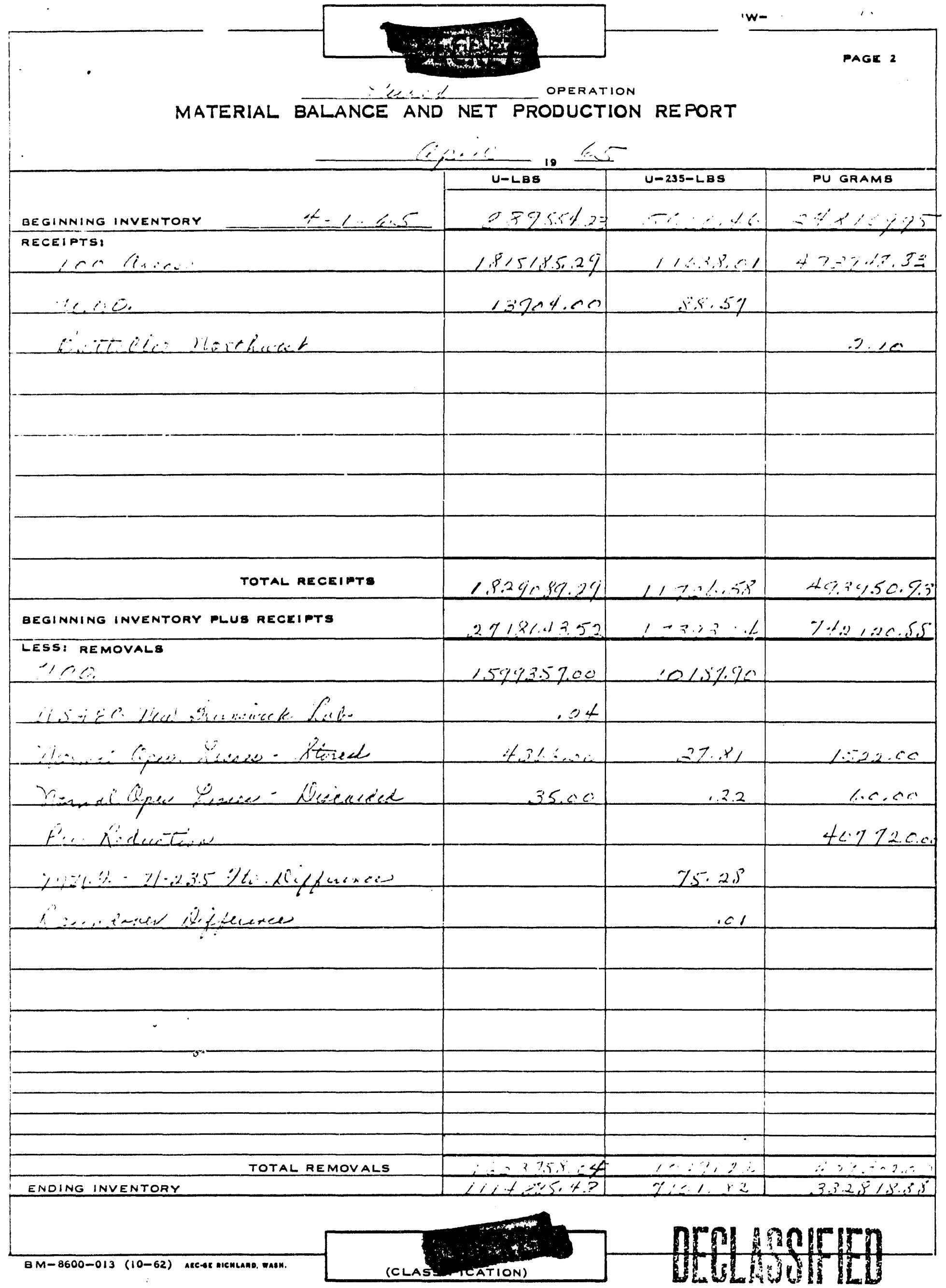




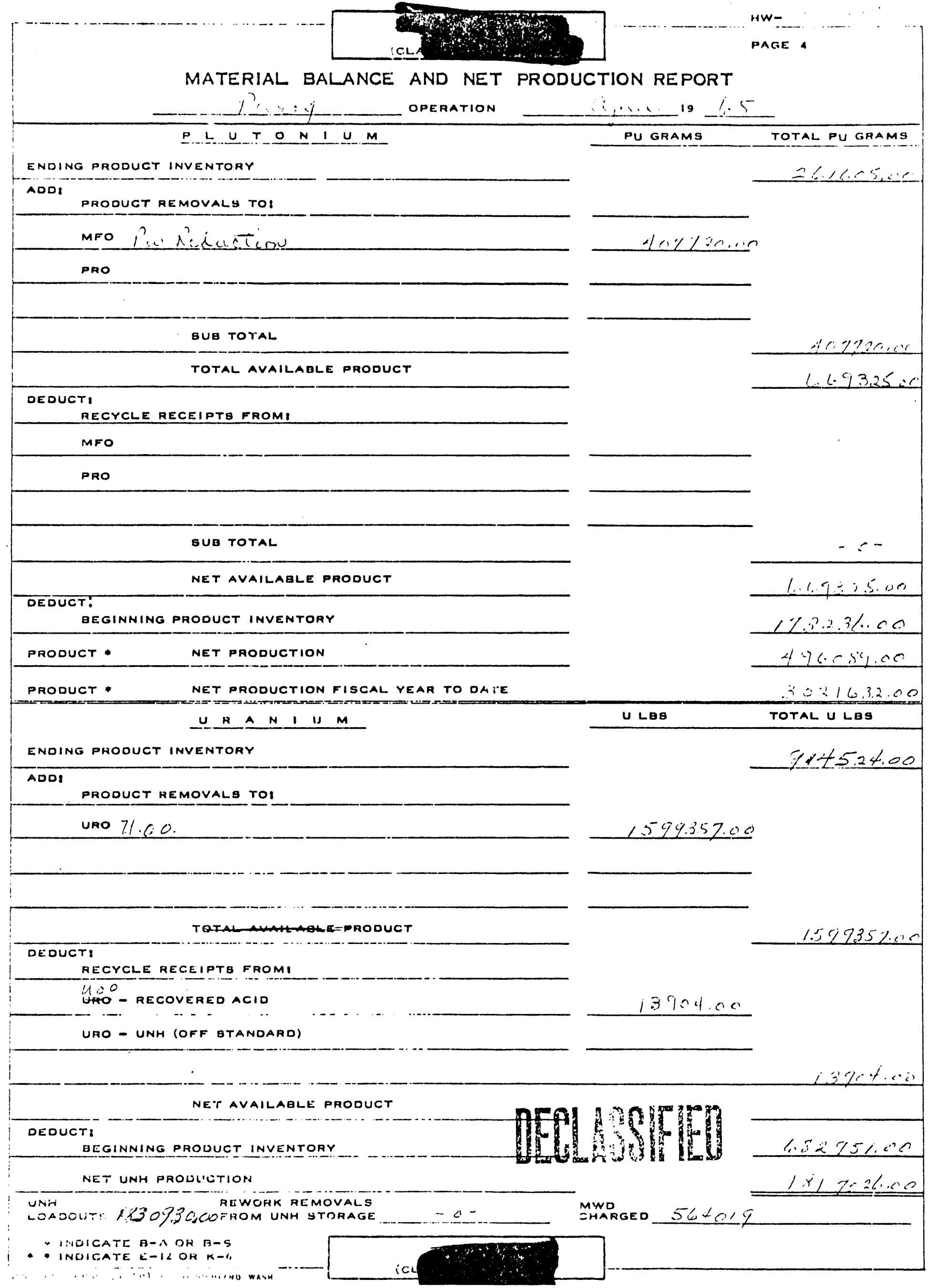




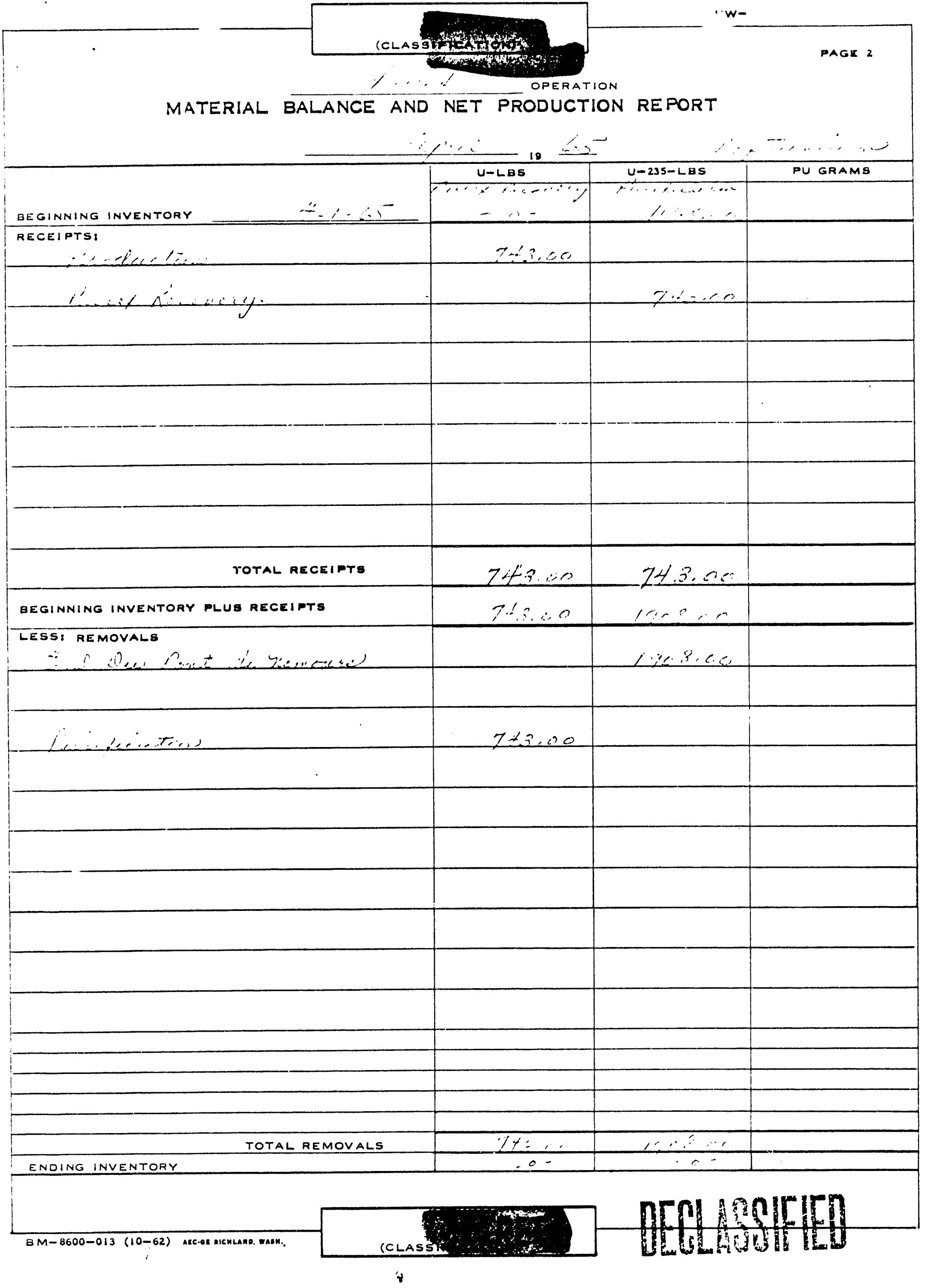




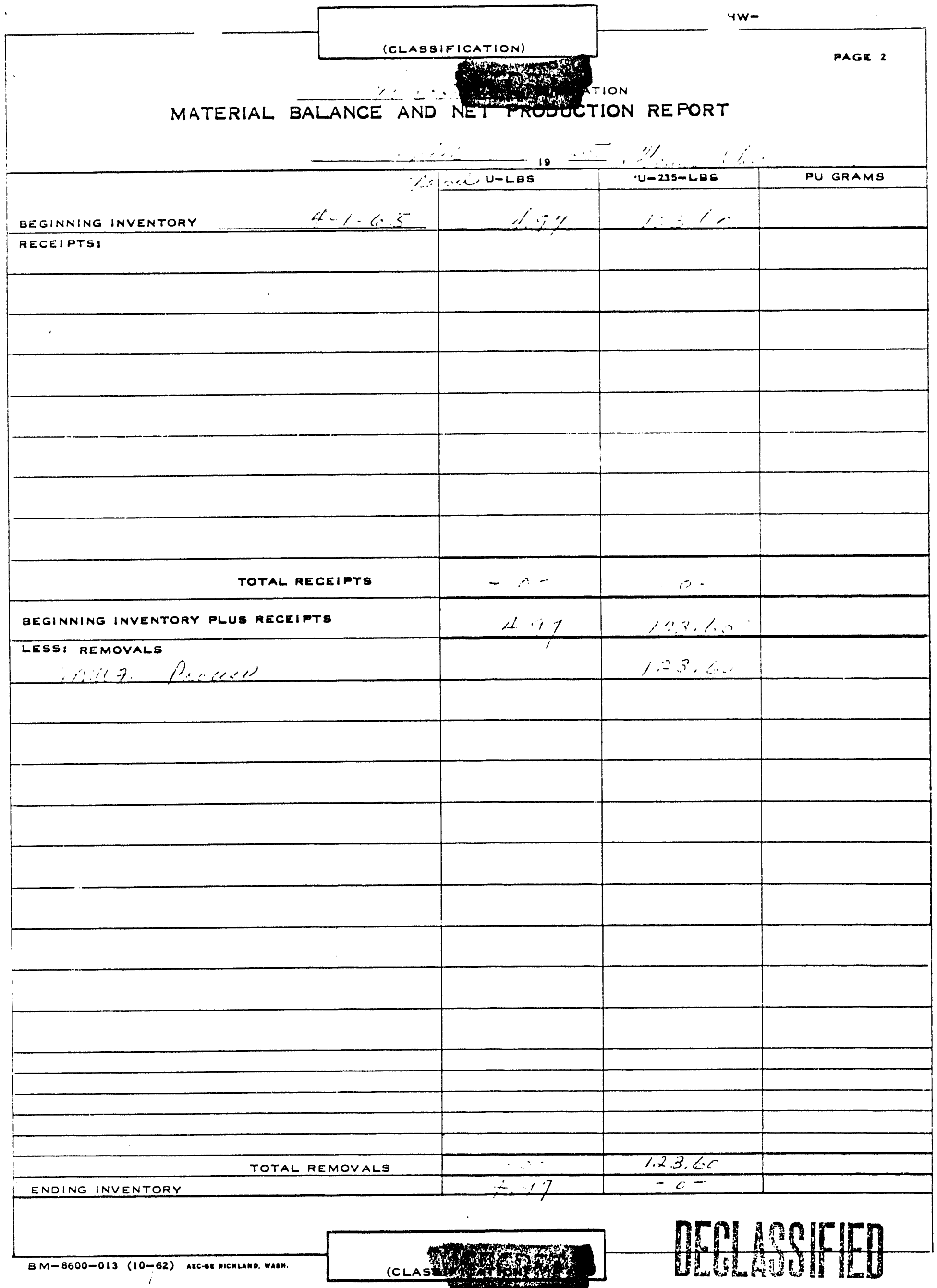




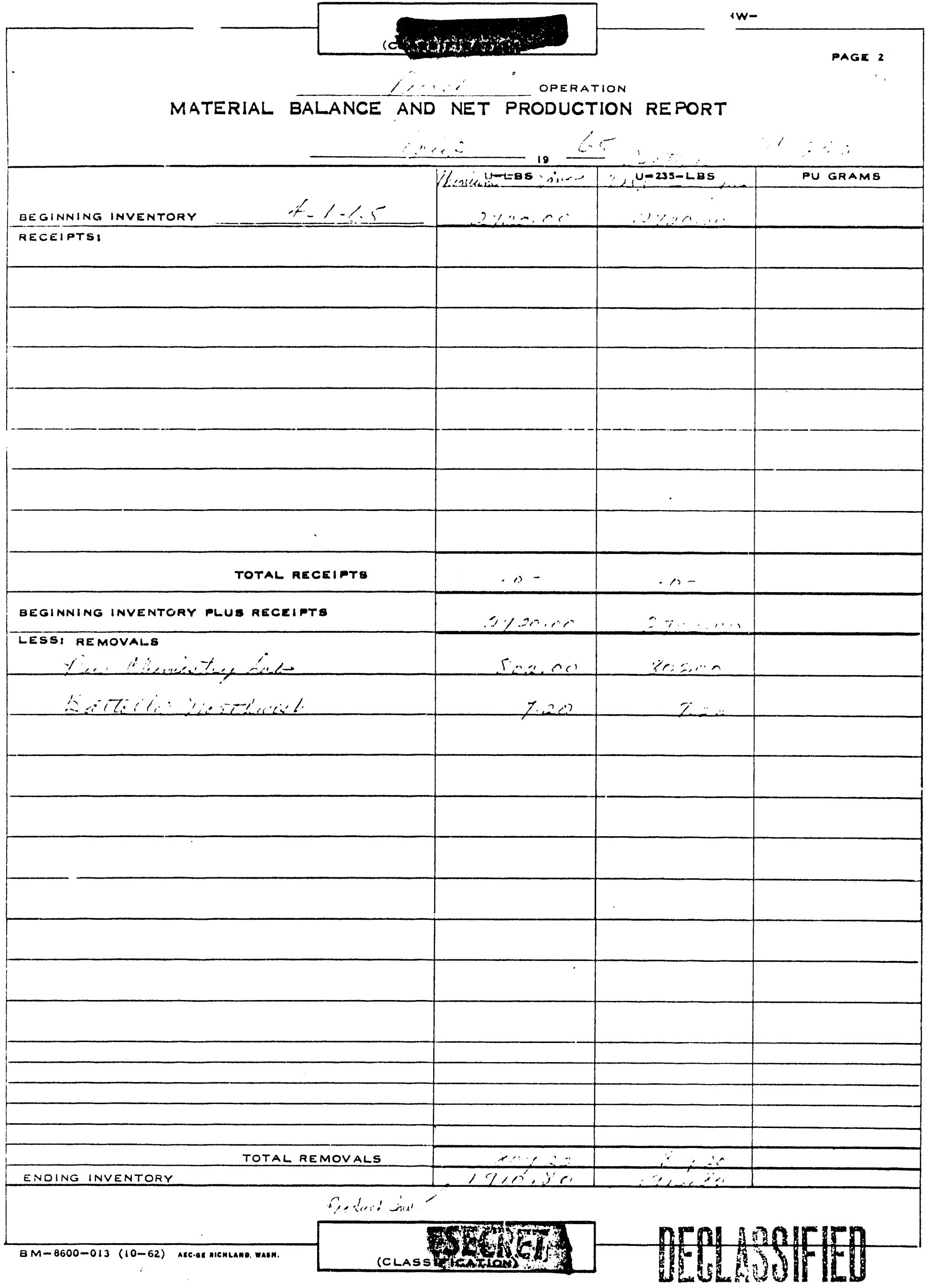




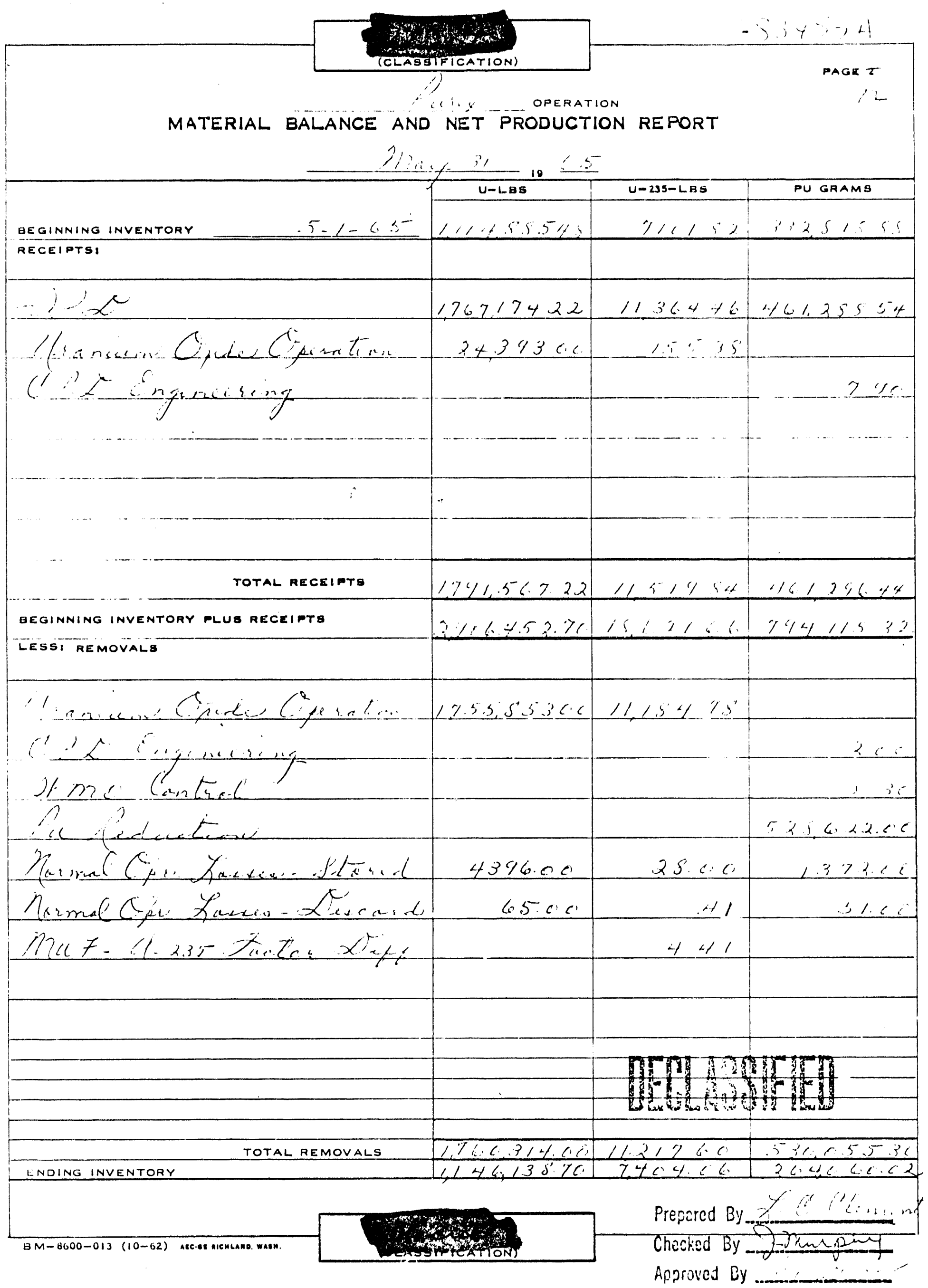




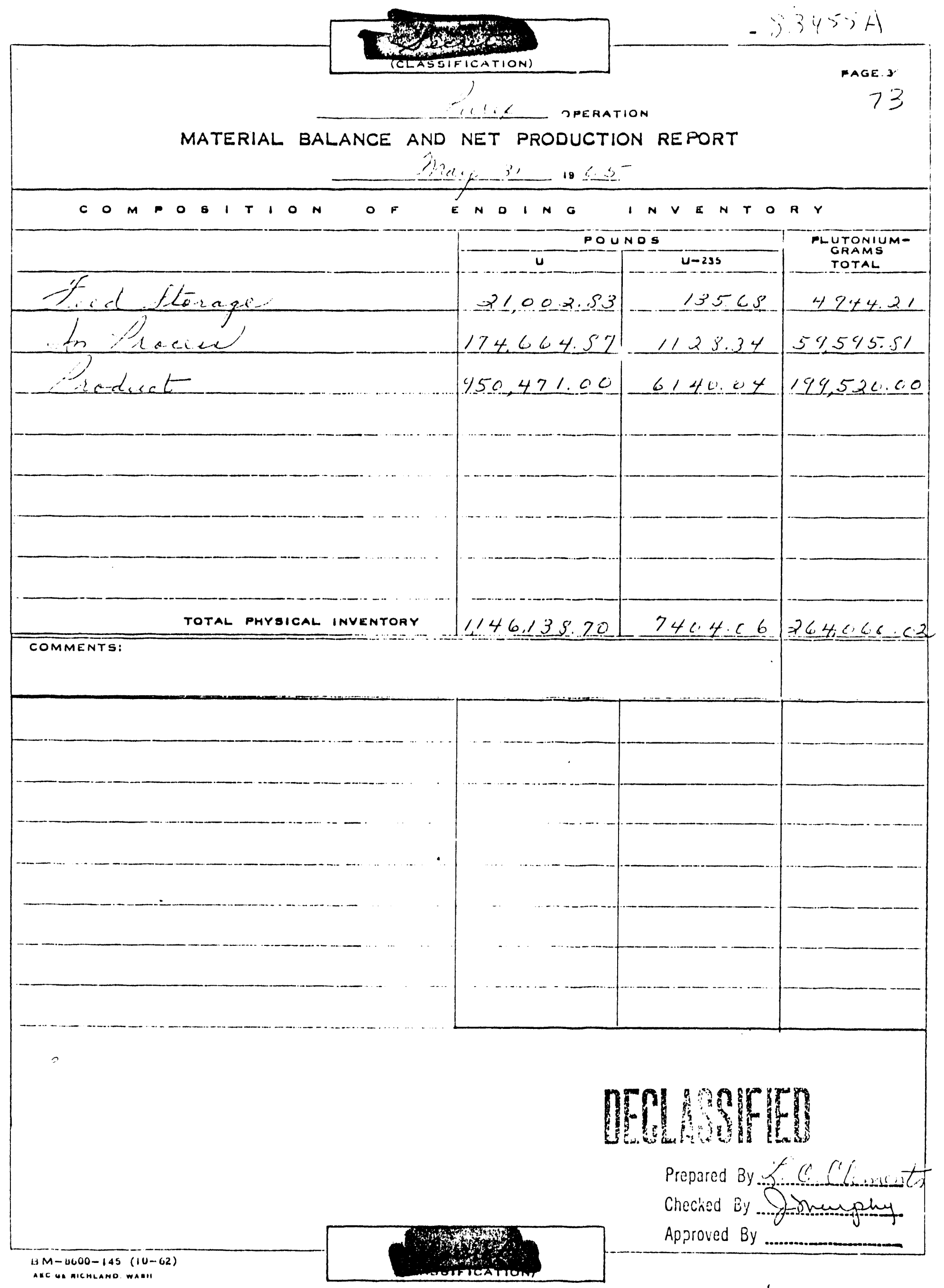




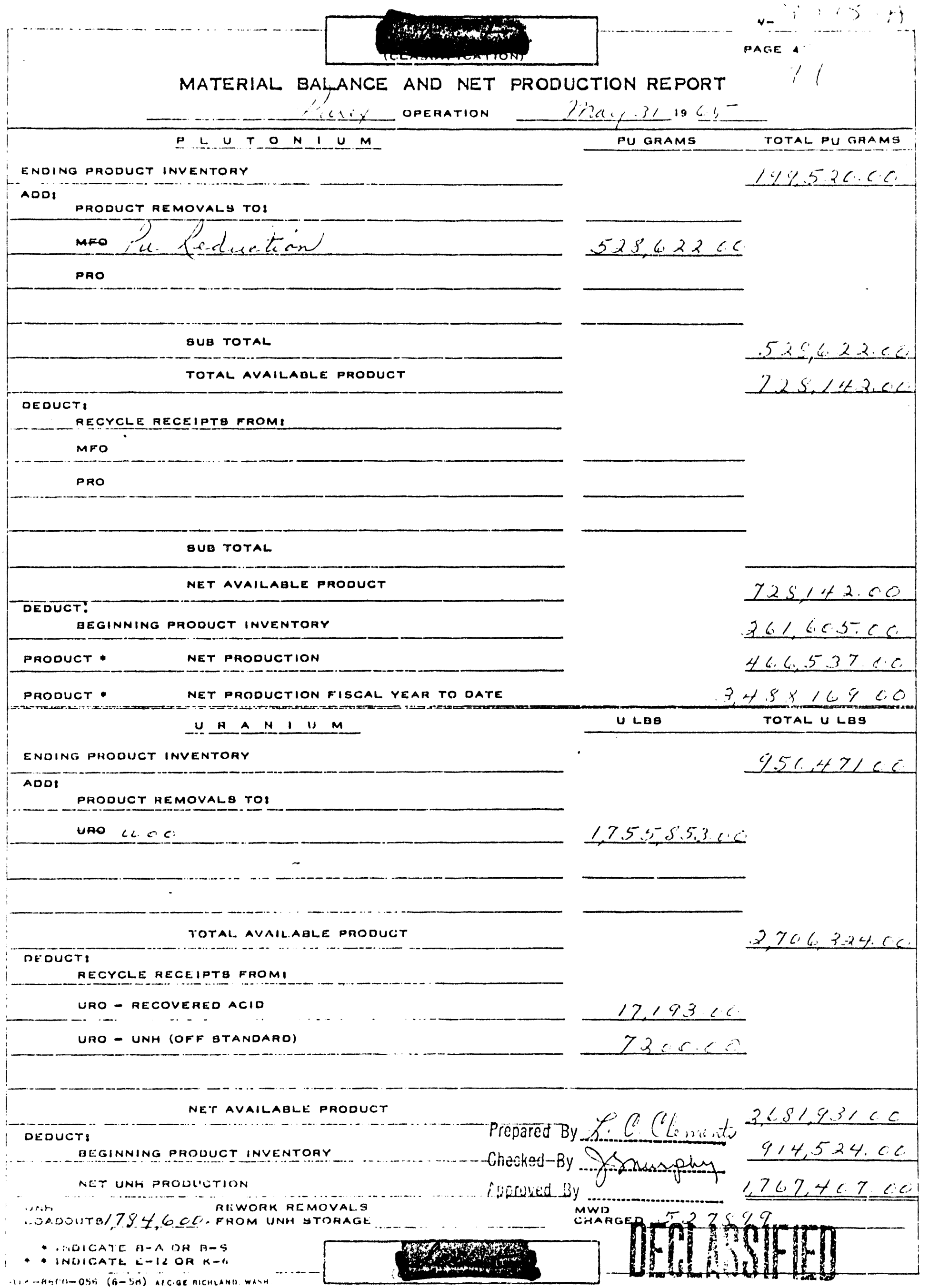




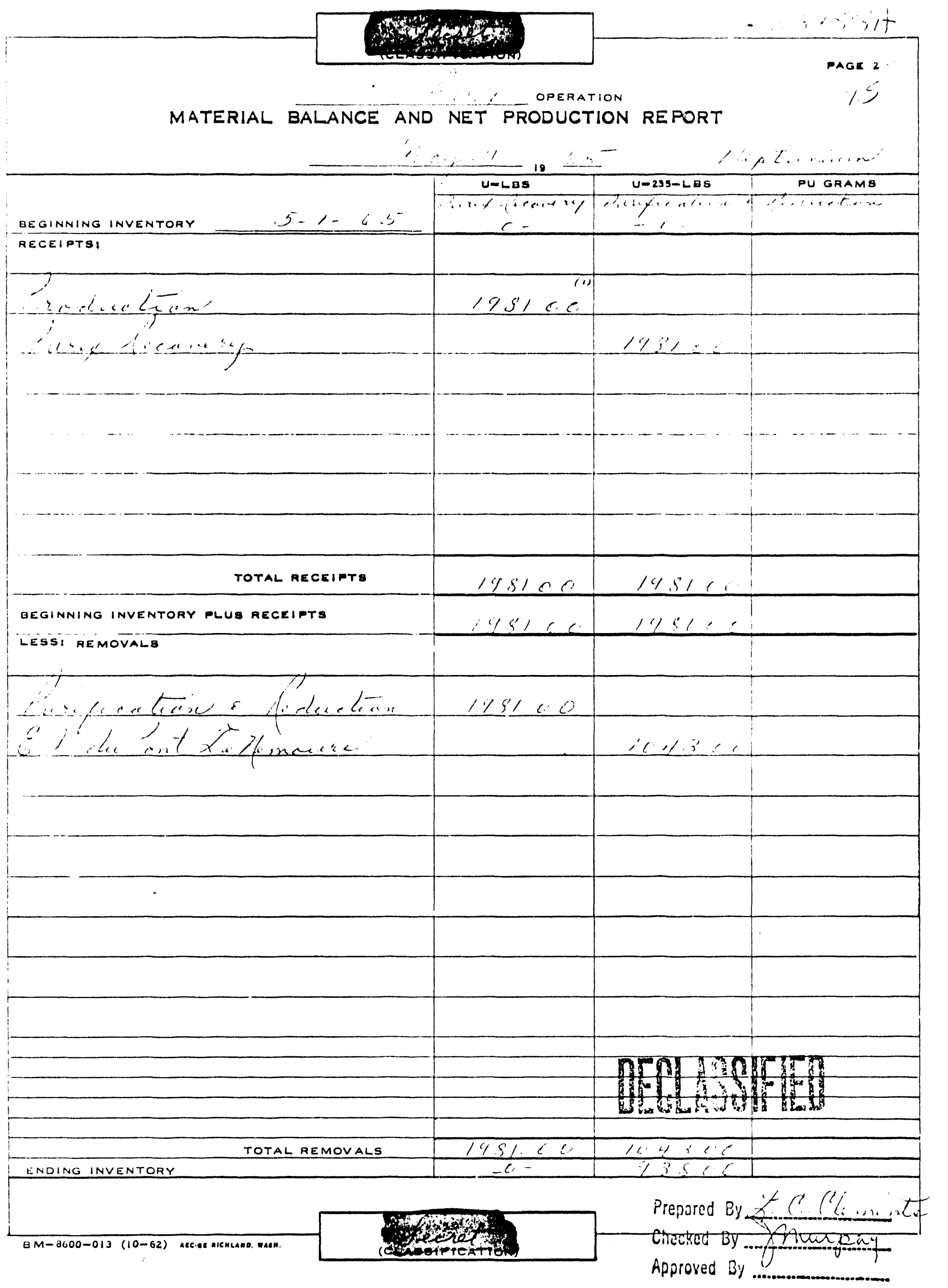




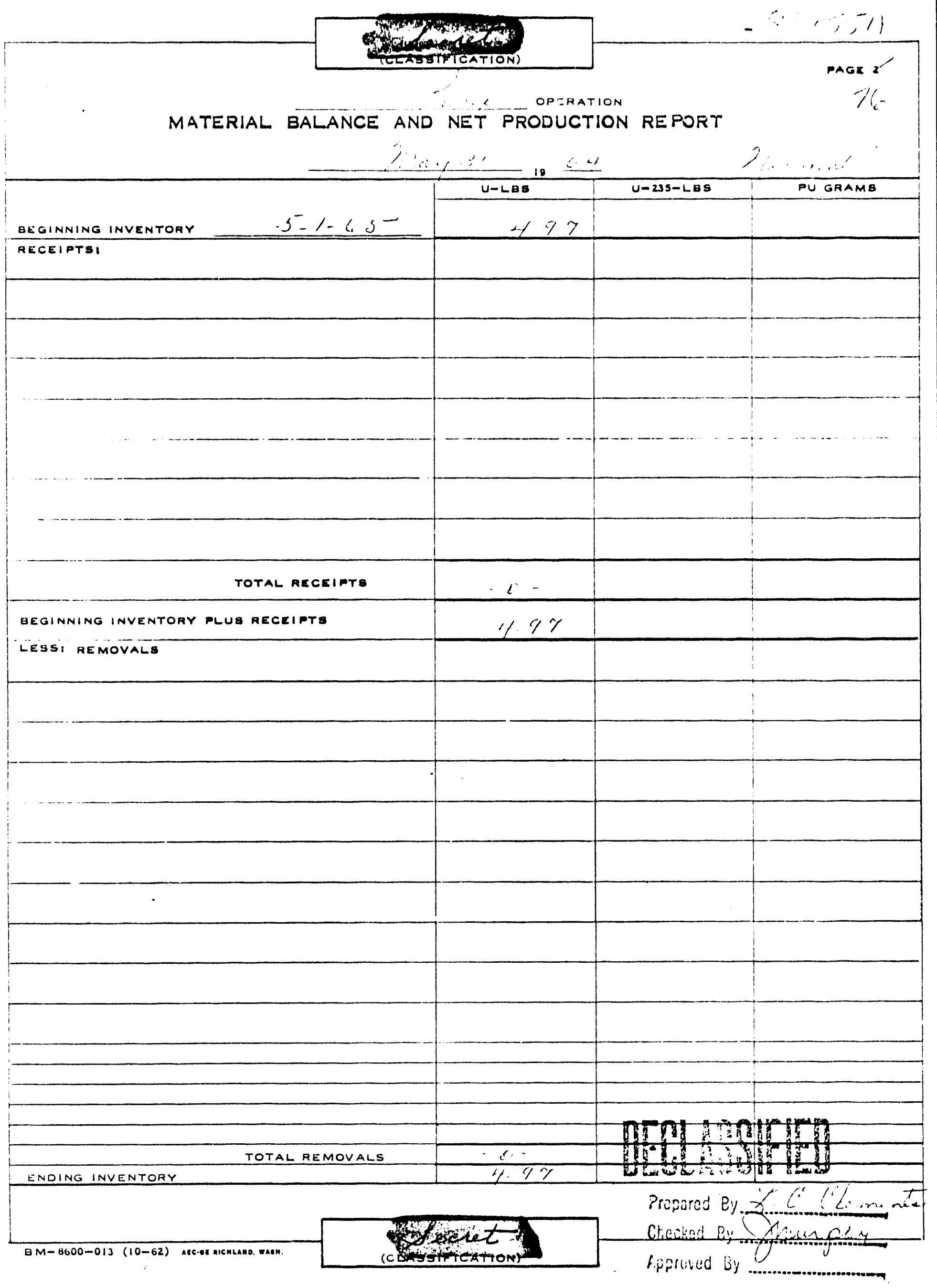




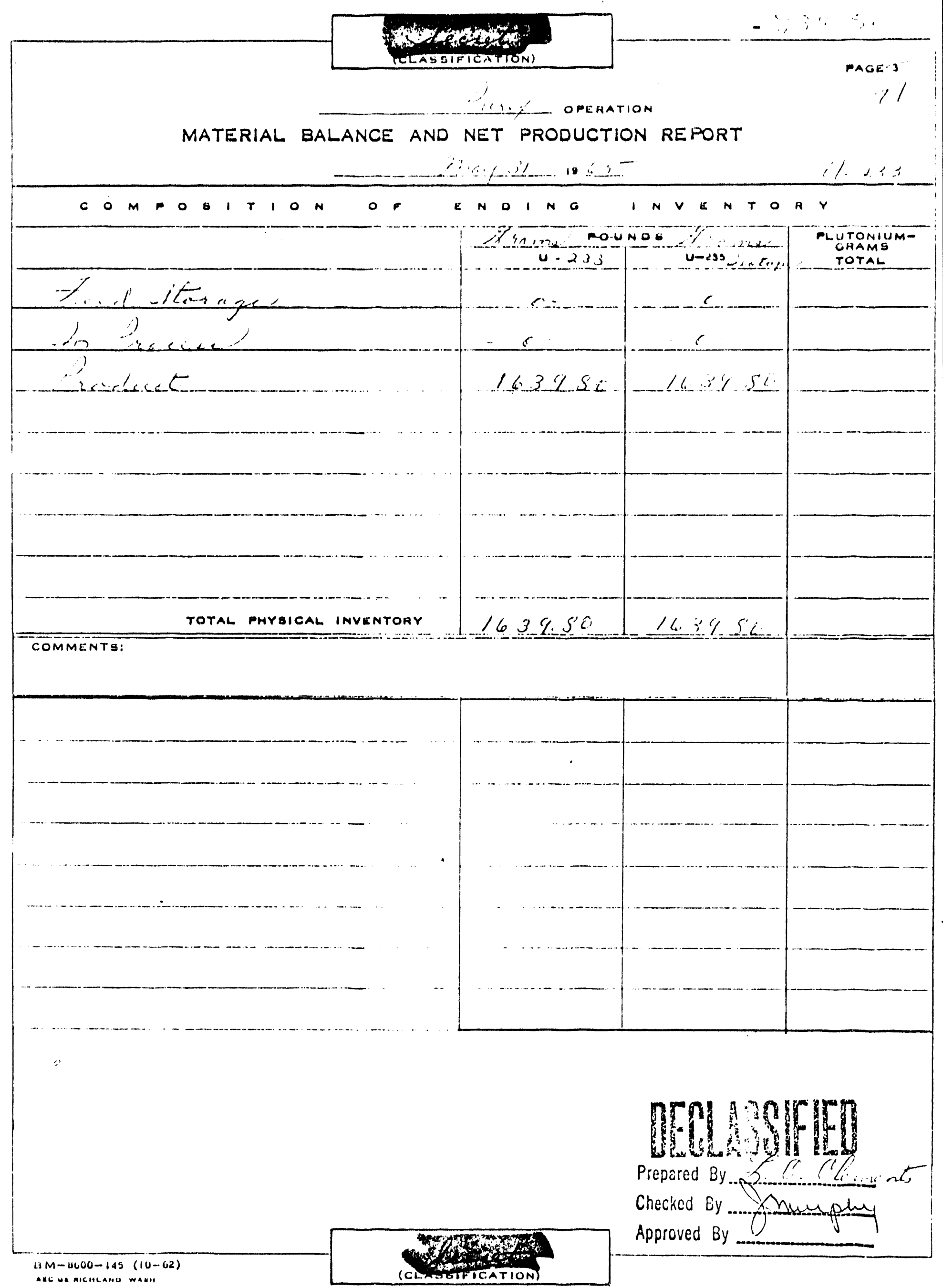




\section{MATERIAL BALANCE AND NET PR̃ODUCTION REPORT}

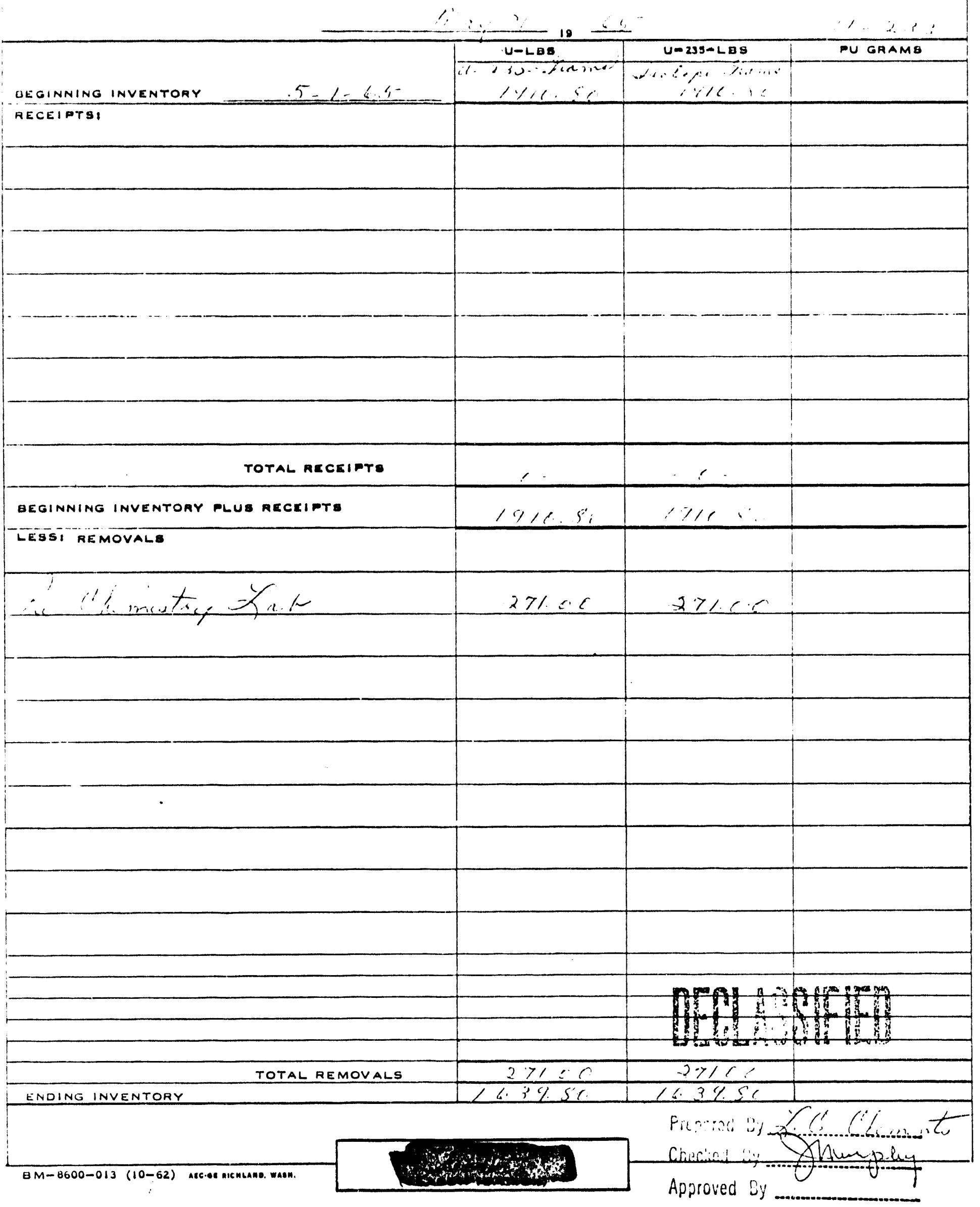




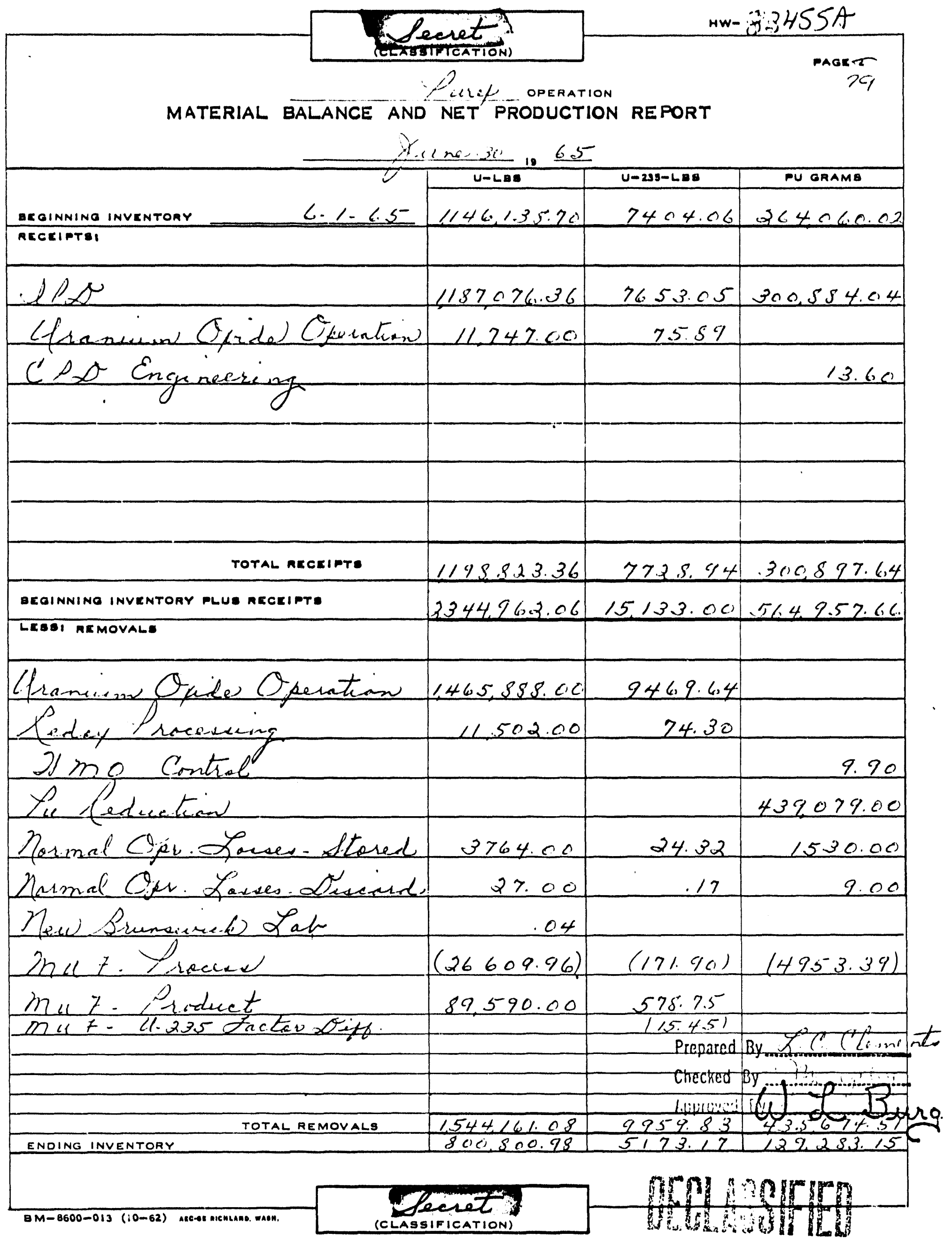




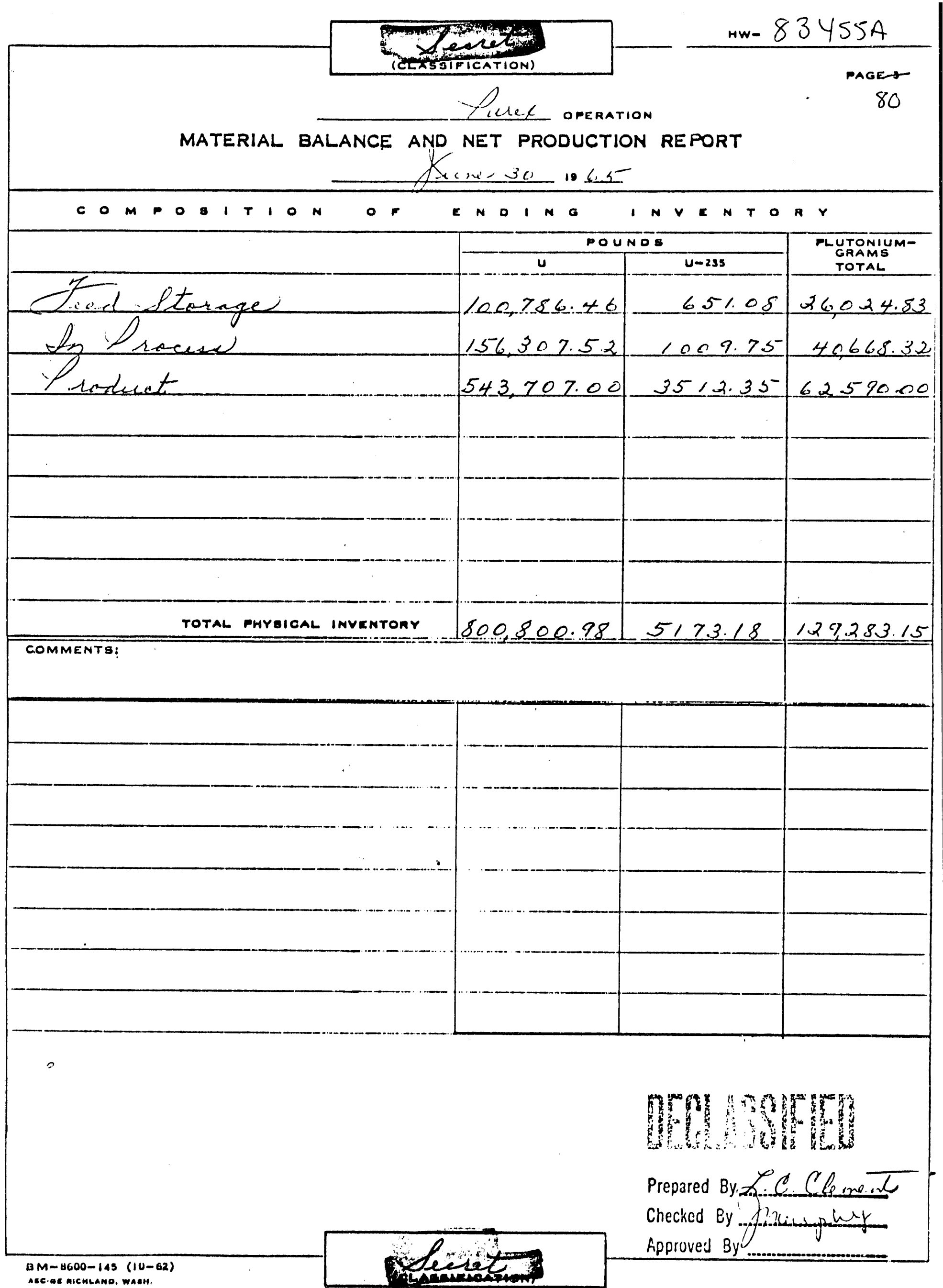




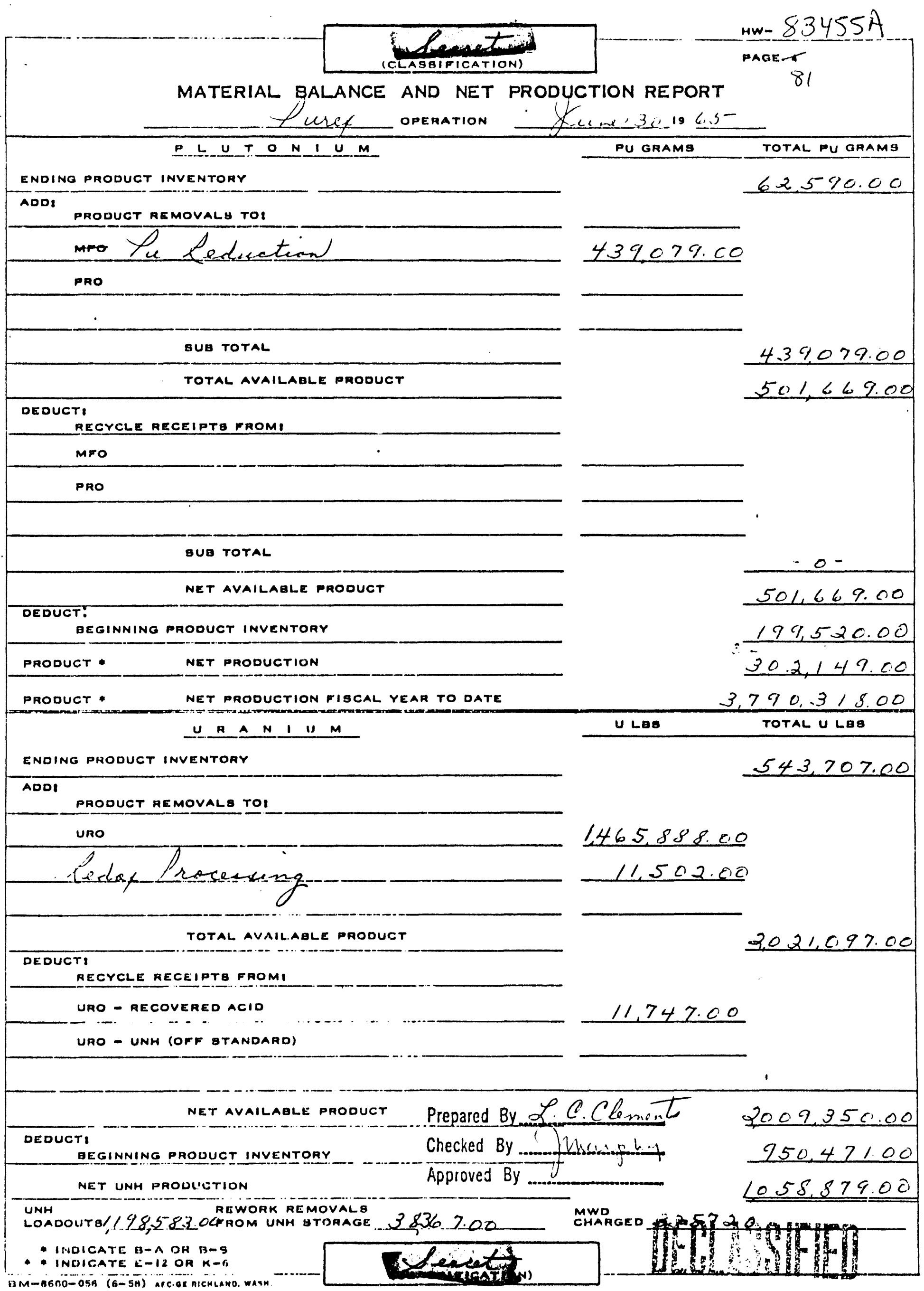




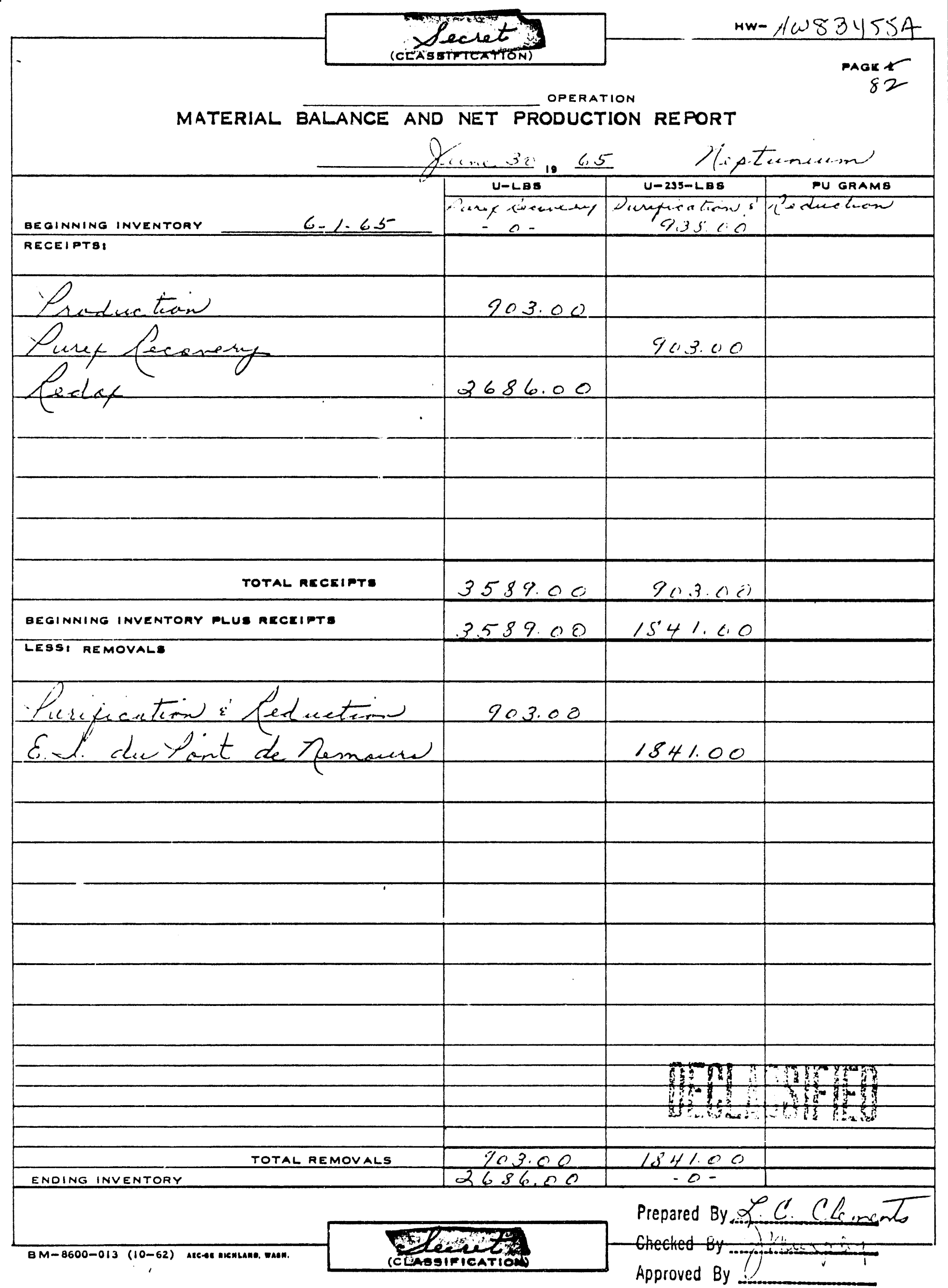




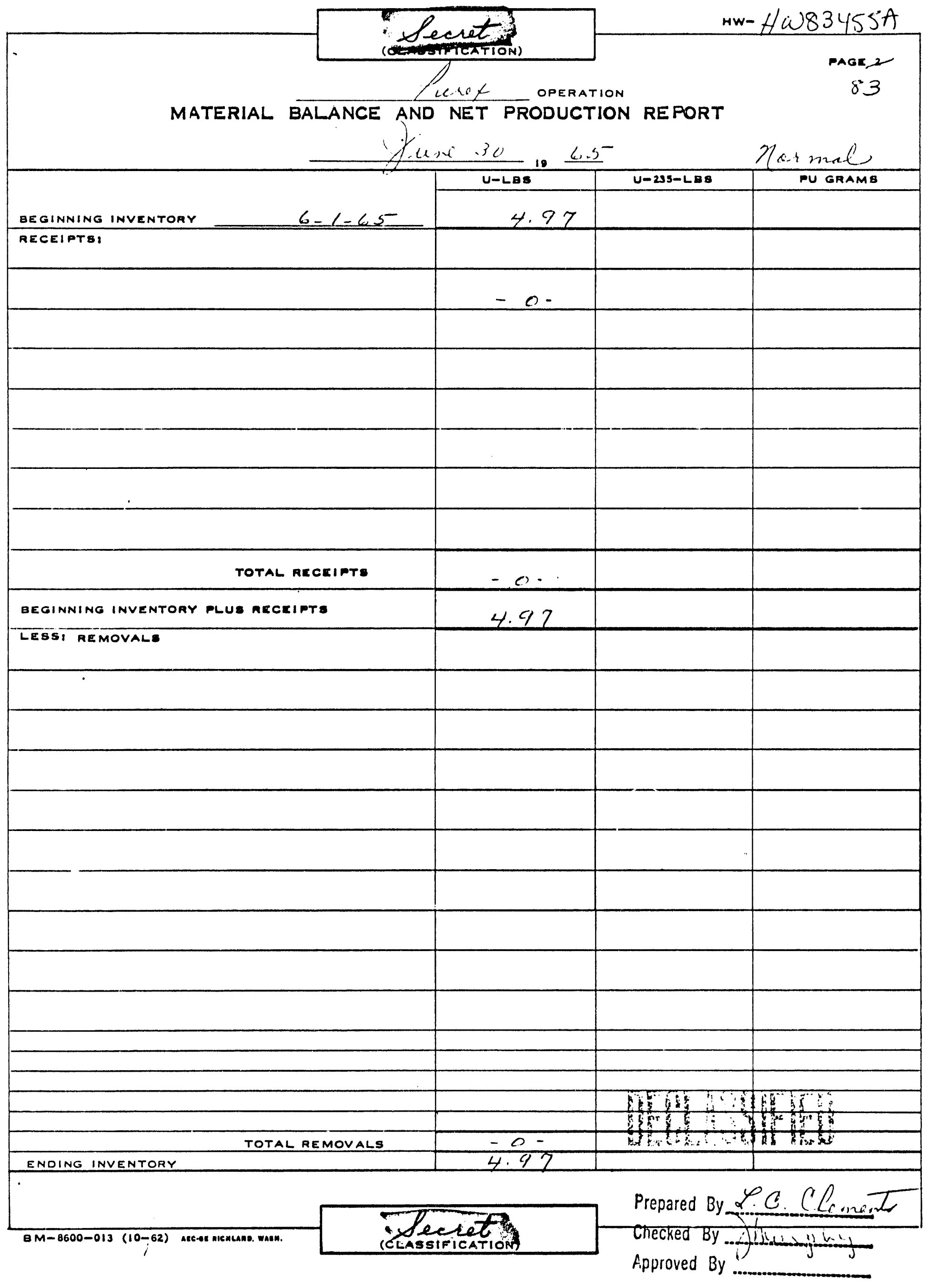




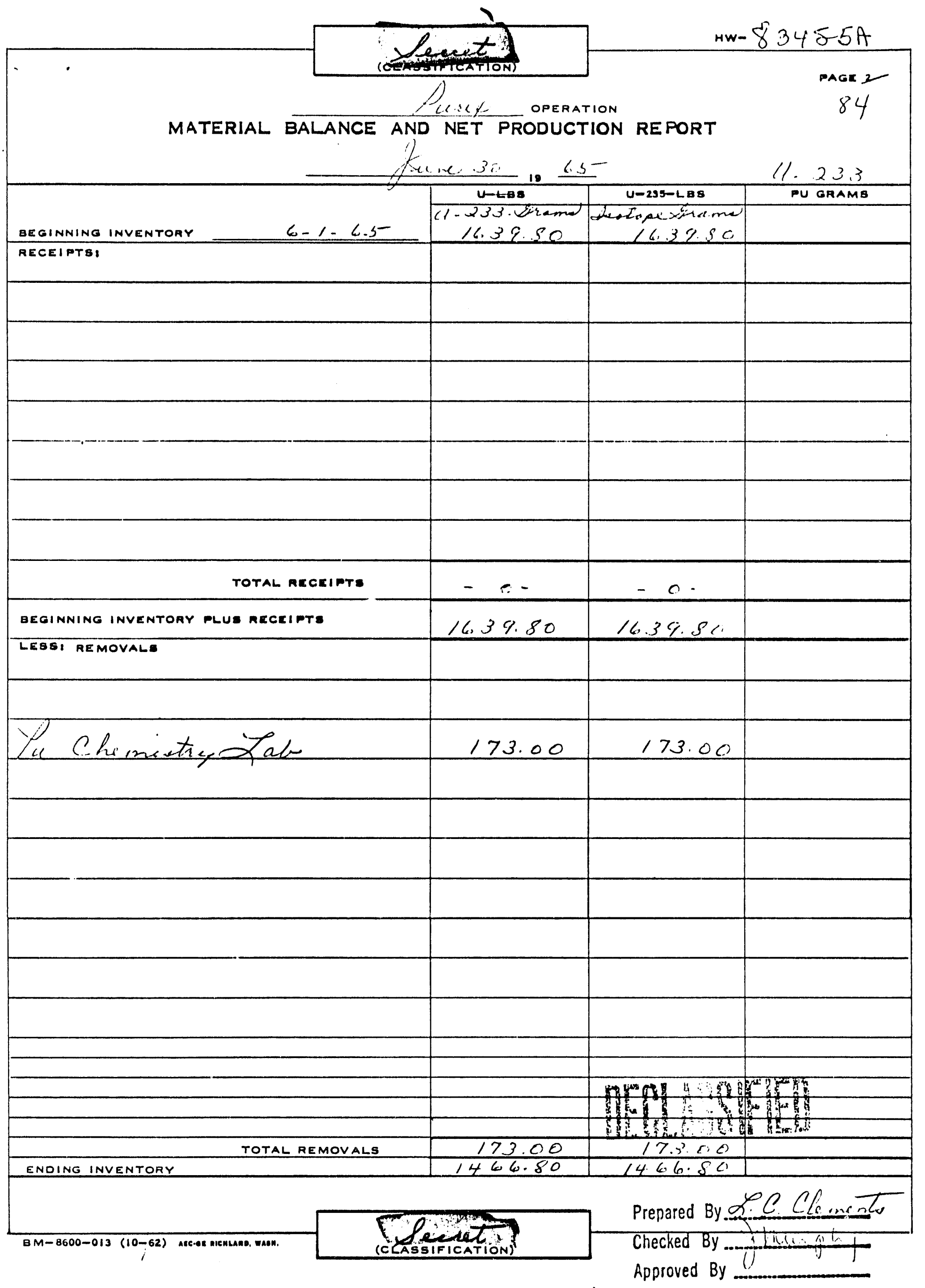


- $\kappa_{2}$ ponosddy

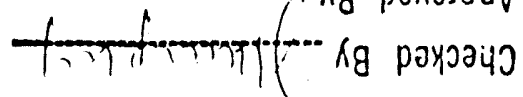

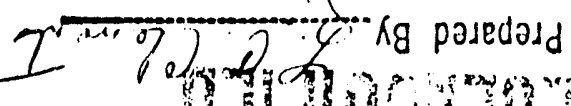
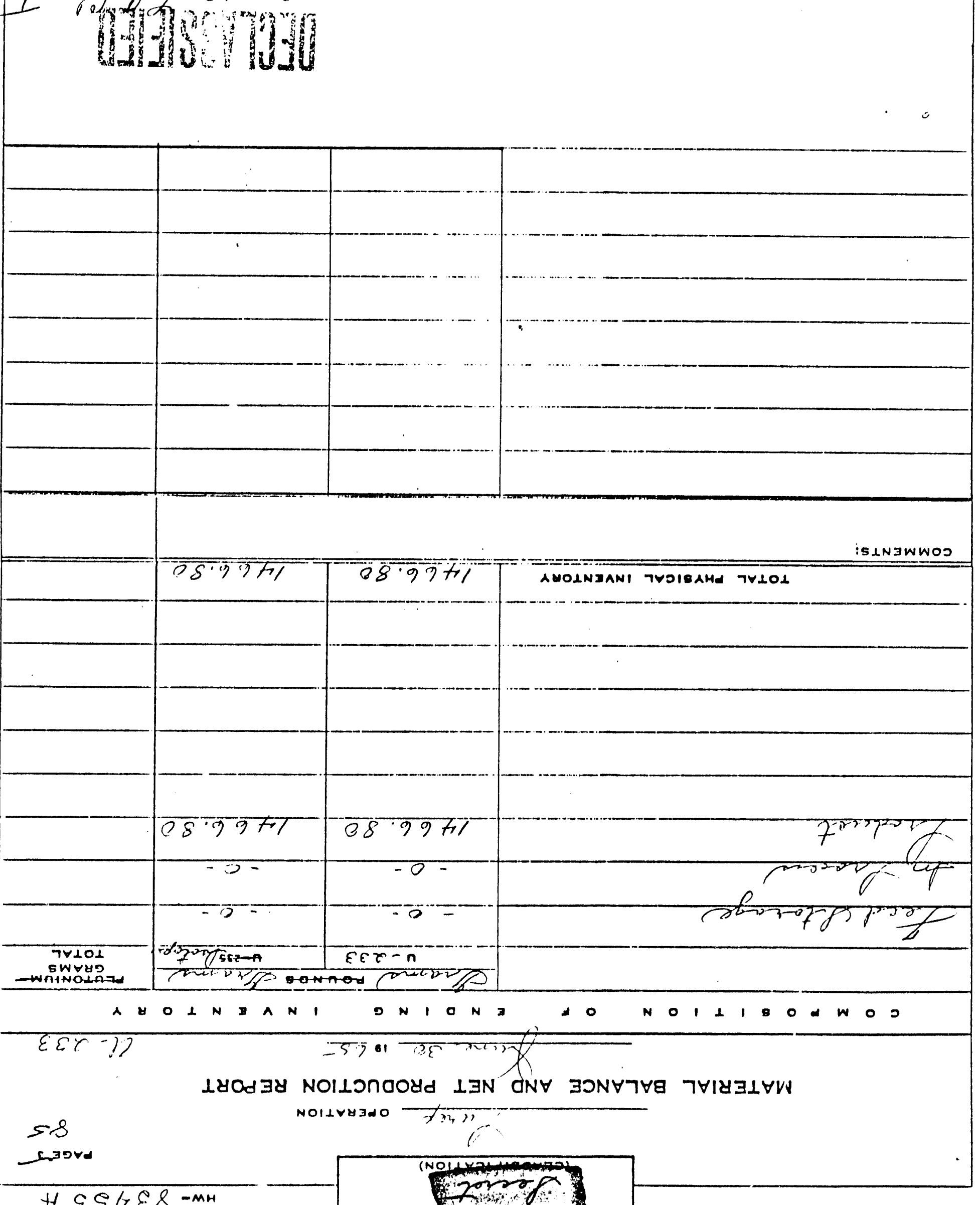

$\forall S S / 7 \& 8^{-M H}$ 

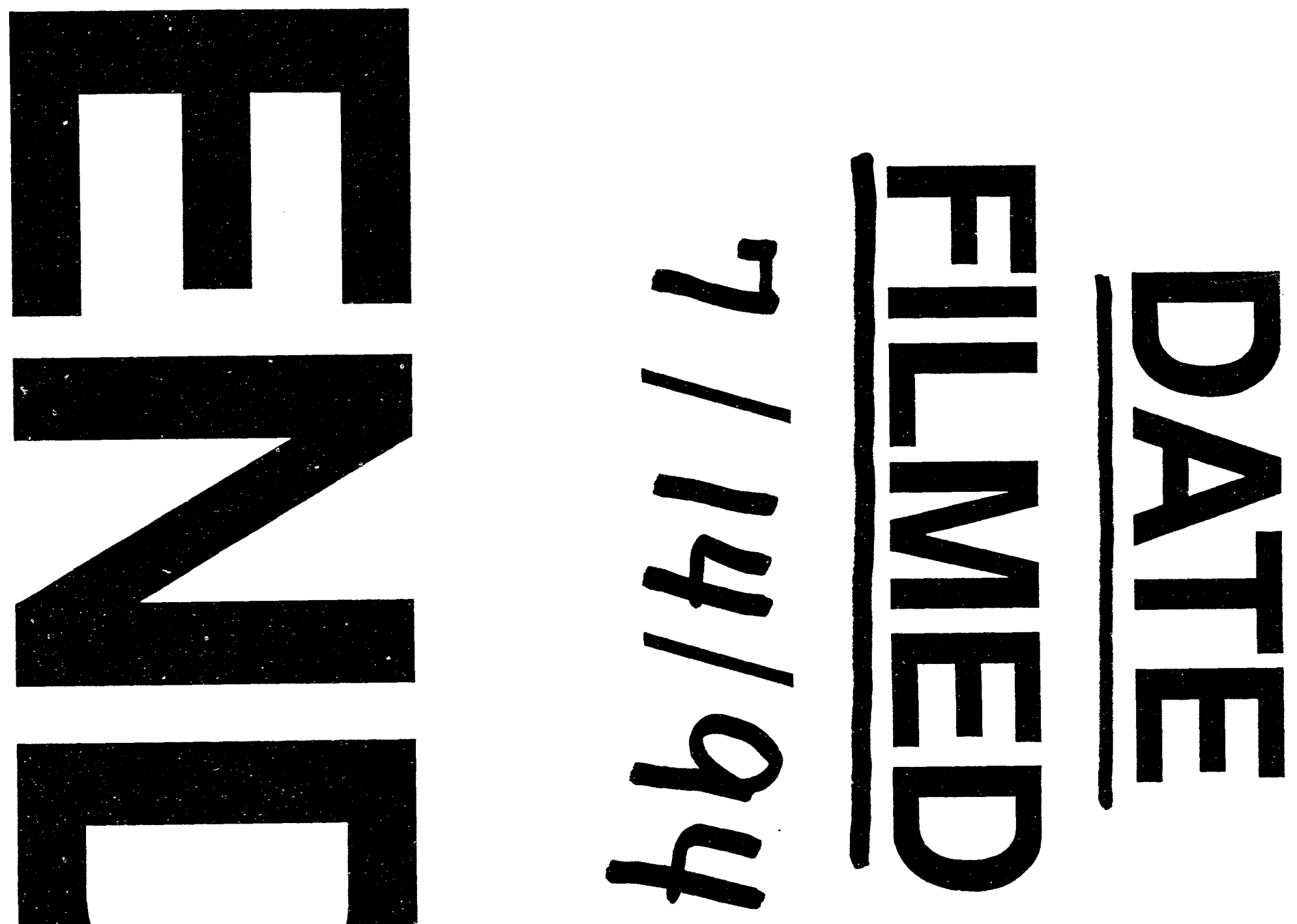
